Sistemas dinâmicos com um único ponto de equilíbrio e injetividade

Jean Venato Santos 


\title{
Sistemas dinâmicos com um único ponto de equilíbrio e injetividade
}

\author{
Jean Venato Santos \\ Orientador: Carlos Alberto Maquera Apaza \\ Co-orientador: José Andrés Martínez Alfaro \\ (Universidad de Valencia)
}

Tese apresentada ao Instituto de Ciências Matemáticas e de Computação - ICMC-USP, como parte dos requisitos para obtenção do título de Doutor em Ciências - Matemática. VERSÃO REVISADA. 
Ficha catalográfica elaborada pela Biblioteca Prof. Achille Bassi e Seção Técnica de Informática, ICMC/USP, com os dados fornecidos pelo(a) autor(a)

\begin{tabular}{|c|c|}
\hline \multirow[t]{3}{*}{ V448s } & $\begin{array}{l}\text { Venato Santos, Jean } \\
\text { Sistemas dinâmicos com um único ponto de equilíbrio } \\
\text { e injetividade / Jean Venato Santos; orientador } \\
\text { Carlos Maquera -- São Carlos, } 2011 . \\
\quad 74 \mathrm{p} \text {. }\end{array}$ \\
\hline & $\begin{array}{l}\text { Tese (Doutorado - Programa de Pós-Graduação em } \\
\text { Ciências de Computação e Matemática Computacional) -- } \\
\text { Instituto de Ciências Matemáticas e de Computação, } \\
\text { Universidade de São Paulo, } 2011 .\end{array}$ \\
\hline & $\begin{array}{l}\text { 1. Sistemas dinâmicos. 2. Injetividade global. I. } \\
\text { Maquera, Carlos, orient. II. Título. }\end{array}$ \\
\hline
\end{tabular}


À minha amada Catiana, companheira fiel na matemática e na vida; Aos meus pais, Carlos e Joana, pelo amor e apoio incondicionais. À minha irmã e à nossa querida Joice. 


\section{AgRAdeCIMENTOS}

Aos meus pais que não mediram esforços para proporcionar todas as condições necessárias para minha formação pessoal e profissional. À minha irmã pelo constante apoio em toda minha vida.

À minha querida Catiana Casonatto, pelo amor, pela doce e suave presença e pelo companheirismo nestes anos de doutorado e por muitos outros que virão.

Aos professores Carlos Maquera e José Martínez Alfaro pela preciosa disponibilidade e excelente orientação na minha iniciação à pesquisa em matemática.

Aos professores do Instituto de Ciências Matemáticas e Computação (ICMC-USP) pelos valiosos ensinamentos. A todos os funcionários deste instituto que proporcionam um ótimo ambiente de trabalho para seus alunos e professores.

Pelo acolhimento, gostaria de agradecer aos professores, funcionários e alunos dos departamentos de Análisis Matemático e de Topología y Geometría da Universidad de Valencia.

Aos alunos da pós-graduação do ICMC-USP, contemporâneos de uma etapa compartilhada. Em especial: Kleyber Cunha (Bahiano), Márcio Fenille, Thiago Catalan (o Rústico), Thaís Monis (Thaizinha), Luiz Hartmann, Flank Bezerra (Capitão), Mário Henrique (Ronaldinho), Mário Henrique (Caverna), Márcio Jorge, Thaís Jordão (Thaizão), Romero Melo, Nazira Harb, Fernando Micena, Walter Vargas, Renato Tintaya, Vinícius Arakawa, Romenique Rocha, Suzete Afonso, John Beiro, Éder Aragão (Edinho), Marcos Pimenta. 


\section{RESUMO}

A primeira parte deste trabalho é dedicada ao estudo de sistemas dinâmicos contínuos e discretos bidimensionais com um único ponto de equilíbrio que é do tipo sela hiperbólica. No caso contínuo, obtemos condições suficientes para que um campo vetorial planar seja topologicamente equivalente à sela linear $L(x, y)=(-x, y)$. No caso em que o campo vetorial é um difeomorfismo local, a injetividade do campo jogará um papel fundamental na obtenção de tal equivalência topológica. Além disto, apresentamos uma descrição das folheações do plano associadas a campos de vetores com uma única singularidade do tipo sela hiperbólica.

No âmbito dos sistemas discretos, apresentamos condições para que um difeomorfismo, possuindo uma sela hiperbólica como único ponto fixo, satisfaça as propriedades básicas de um sistema linear com um ponto fixo que é do tipo sela hiperbólica: as quatro separatrizes do ponto fixo se acumulam só no infinito e os iterados dos pontos que não estão nas variedades invariantes deste ponto fixo se acumulam no infinito tanto no passado quanto no futuro.

A segunda parte deste texto, se dedica a problemas de injetividade de difeomorfismos locais em $\mathbb{R}^{n}$. Mais especificamente, obtemos versões fracas da Conjetura Jacobiana Real de Jelonek e de uma Conjetura apresentada por Nollet e Xavier. Ambos problemas estão intimamente ligados à famosa Conjetura Jacobiana, que foi considerada por Smale em 1998 como um dos dezoito problemas matemáticos mais relevantes ainda em aberto. 


\section{ABSTRACT}

The first part of this work is dedicated to the study of continuous and discrete twodimensional dynamical systems with a unique equilibrium point which is a hyperbolic saddle. In the continuous case, we obtain sufficient conditions for a planar vector field be topologically equivalent to the linear saddle $L(x, y)=(-x, y)$. In the case where the vector field is a local diffeomorphism, the injectivity of the field will play a key role in obtaining such a topological equivalence. Furthermore, we provide a description of foliations of the plane vector fields associated with a unique singularity of hyperbolic saddle type.

In the context of discrete systems, we present conditions for a diffeomorphism, possessing a hyperbolic saddle as the single fixed point, to satisfy the basic properties of a linear system with a fixed point of saddle type which is hyperbolic: the four separatrices of the fixed point accumulate only at infinity and iterated the points that are not in invariant manifolds of this fixed point accumulate in infinity in both the past and future.

The second part of this text is devoted to problems of injectivity of local diffeomorphisms on $\mathbb{R}^{n}$. More specifically, we obtain weaker versions of the Jelonek's Real Jacobian Conjecture and a Conjecture given by Nollet and Xavier. Both problems are closely linked to the famous Jacobian Conjecture, which was considered by Smale in 1998 as one of eighteen mathematical problems even more important in open. 


\section{CONTEÚdo}

Introdução 1

\begin{tabular}{lll}
\hline 1 & Preliminares & 9
\end{tabular}

1.1 Campos vetoriais $\ldots \ldots \ldots \ldots \ldots$

1.1 .1 Campos vetoriais planares . . . . . . . . . . . . . . . . . . . 11

1.2 Folheações $\ldots \ldots \ldots \ldots$. . . . . . . . . . . . . . . . . . . . . . . . 13

1.2 .1 Folheações do plano . . . . . . . . . . . . . . . . . . . . . 18

1.3 Sistemas discretos $\ldots \ldots \ldots$. . . . . . . . . . . . . . . . . 20

2 Sistemas dinâmicos bidimensionais com um único ponto de equilíbrio 23

2.1 Caso contínuo . . . . . . . . . . . . . . . . . . . . . . . . 23

2.1 .1 Sela global . . . . . . . . . . . . . . . . . . . . . 23

2.1 .2 Sela global e injetividade . . . . . . . . . . . . . . . . . . . . . . . . 29

2.1 .3 Exemplos . . . . . . . . . . . . . . . . . . . . 33

$2.1 .4 \quad$ Folheações do plano associadas a $X \in \mathfrak{X}_{s}^{1}\left(\mathbb{R}^{2}\right) \ldots \ldots$. . . . . . . . 37

2.2 Caso discreto $\ldots \ldots \ldots \ldots \ldots \ldots$. . . . . . . . . . . . . . . . . . . . . . 44 
\begin{tabular}{lll}
\hline 3 & Injetividade Global & 49
\end{tabular}

3.1 Difeomorfismos locais e folheações . . . . . . . . . . . . . . . . . . . . . . . 49

3.2 Versão fraca da Conjetura Jacobiana real de Jelonek . . . . . . . . . . . . 51

3.3 Versão fraca da Conjetura de Nollet e Xavier . . . . . . . . . . . . . . . . . 61

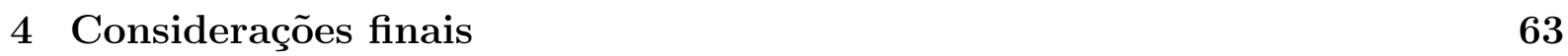

\begin{tabular}{ll}
\hline Bibliografia & 67 \\
\hline
\end{tabular}

\begin{tabular}{ll}
\hline Índice & 73
\end{tabular} 


\section{INTRODUÇÃO}

Este trabalho se insere no contexto dos sistemas dinâmicos tanto contínuos quanto discretos. Dado um espaço topológico $M$ e $(G,$.$) um semigrupo topológico. Dizemos que$ um sistema dinâmico é um par $(M, A)$, onde $A: M \times G \rightarrow M$ é uma aplicação contínua que satisfaz:

- $A(A(p, g), h)=A(p, g . h)$, se $p \in M, g, h \in G$, e

- $A(p, e)=p$, onde $p \in M$ e $e$ é o elemento neutro de $G$.

No caso em que $G=\mathbb{R}$, dizemos que $(M, A)$ é um sistema dinâmico contínuo. No caso em que $G=\mathbb{N}$ ou $\mathbb{Z}$, dizemos que $(M, A)$ é um sistema dinâmico discreto. Geralmente os sistemas dinâmicos contínuos são, quase sempre, definidos quando $M$ é uma variedade suave, e $A$ é um fluxo definido a partir de um campo vetorial diferenciável sobre $M$. Já os sistemas dinâmicos discretos são definidos da seguinte maneira: se $f: M \rightarrow M$ é um homeomorfismo de um espaço topológico nele mesmo, definimos $A(p, k)=f^{k}(p)$, onde $p \in M$, e $k \in \mathbb{Z}$. Se um ponto $p \in M$ satisfaz $A(p, g)=p$ para todo $g \in G$, dizemos que $p$ é um ponto de equilíbrio do sistema dinâmico $(M, A)$. No caso de sistemas contínuos tais pontos também são chamados de pontos singulares e no caso discreto pontos fixos. Neste trabalho consideraremos $M=\mathbb{R}^{n}$ e, em particular, estamos interessados nos sistemas com um único ponto de equilíbrio.

No que segue apresentaremos nossos principais resultados, para isto se faz necessária a introdução de certas terminologias.

Dada uma aplicação $X: \mathbb{R}^{n} \rightarrow \mathbb{R}^{n}$, de classe $C^{1}$, denote por $\operatorname{Spec}(X)$ o conjunto dos autovalores da derivada $D X_{p}$ quando $p$ varia em todo $\mathbb{R}^{n}$. Em grande parte deste trabalho, estaremos interessados em estudar as relações entre certas condições sobre o conjunto $\operatorname{Spec}(X)$, a injetividade da aplicação $X$ e a dinâmica do sistema contínuo $x^{\prime}=$ $X(x)$, associado ao campo vetorial $X$. No caso unidimensional, por exemplo, a condição 
$\operatorname{Spec}(X) \cap\{0\}=\varnothing$ implica que $X^{\prime}(p) \neq 0$, isto é, $X^{\prime}(p)<0$ ou $X^{\prime}(p)>0$, para todo $p \in \mathbb{R}$. Assim, a aplicação $X: \mathbb{R} \rightarrow \mathbb{R}$ é estritamente monótona e, em particular, injetiva. Além disto, se $X(0)=0$ o sistema $x^{\prime}=X(x)$ possuirá um atrator (resp. repulsor) global em 0 se $X^{\prime}(0)<0$ (resp. $\left.X^{\prime}(0)>0\right)$.

É claro que para $n \geq 2$ a situação é bem diferente e só o fato $0 \notin \operatorname{Spec}(X)$ não é suficiente para concluir nem mesmo a injetividade de $X$. Assim como, não é possível extrair informações qualitativas contundentes sobre a dinâmica do sistema $x^{\prime}=X(x)$ somente com este dado. Portanto, novas hipóteses devem ser agregadas a fim de obter consequências análogas ao caso $n=1$. Por exemplo, no caso bidimensional, Gutierrez em 27] provou que se $\operatorname{Spec}(X) \cap[0, \infty)=\varnothing$ então $X$ é injetiva. Além disso, ele aplicou este resultado para provar o caso bidimensional da seguinte:

Conjetura de Markus-Yamabe: Seja $X: \mathbb{R}^{n} \rightarrow \mathbb{R}^{n}$ um campo vetorial de classe $C^{1}$ com um ponto singular na origem $O$ de $\mathbb{R}^{n}$, ou seja, $X(O)=O$. Se

$$
\operatorname{Spec}(X) \subset\{z \in \mathbb{C} ; \operatorname{Re}(z)<0\}
$$

então $O$ é um atrator global do sistema $x^{\prime}=X(x)$, isto é, cada solução deste sistema tende a $O$ quando $t \rightarrow+\infty$.

Este problema foi proposto por Markus e Yamabe em 1960 no trabalho [40], onde os autores obtiveram uma resposta afirmativa para alguns casos particulares. Note que esta conjetura é um problema de estabilidade global do ponto singular $O$ do campo vetorial $X$. Pelo Teorema de Hartman-Grobman, desde que os autovalores da derivada $D X_{O}$ têm todos parte real negativa, segue que toda solução do sistema $x^{\prime}=X(x)$ iniciando numa vizinhança de $O$ tende à origem quando $t \rightarrow+\infty$. A conjetura propõe que tal vizinhança pode ser expandida para todo $\mathbb{R}^{n}$ sempre que $\operatorname{Spec}(X)$ satisfaz 0.1 .

Voltando ao caso bidimensional, em 1963 Olech provou em [46] que a conjetura de Markus-Yamabe era equivalente à seguinte conjetura sobre a injetividade de $X$ : a hipótese espectral (0.1) do problema de Markus-Yamabe implica que $X: \mathbb{R}^{2} \rightarrow \mathbb{R}^{2}$ é uma aplicação injetiva. Posteriormente, surgiram vários resultados parciais, porém uma resposta definitiva só foi apresentada em 1995, por Fessler [23], Gutierrez [27] e Glutsyuk [26] os quais deram, de forma independente, uma resposta afirmativa para esta conjetura (no caso $n=2$ ). Na realidade, os três autores provaram a tal conjetura via equivalência de Olech, ou seja, estabelecendo a injetividade da aplicação $X$.

Ainda na década de 90, foram apresentados contra-exemplos da conjetura acima para $n \geq 3$. De fato, em 1994 Bernat e Llibre apresentaram em [6] contra-exemplos analíticos para $n \geq 4$. E somente em 1997 a conjetura foi totalmente resolvida quando Cima et al, 
apresentaram em [13] um contra-exemplo polinomial para $n \geq 3$. Este último exemplo mostra também que a relação entre injetividade e o fato de ser $O$ um atrator global obtida por Olech no caso bidimensional da conjetura de Markus-Yamabe, é perdida quando $n \geq 3$. De fato, o exemplo polinomial que os autores apresentam é injetivo, a despeito da origem não ser um atrator global. Vale lembrar também que nos trabalhos [19, 41], os autores provaram que uma resposta afirmativa da conjetura de Markus-Yamabe implicaria numa resposta afirmativa para a famosa conjetura Jacobiana, da qual falaremos mais adiante.

A discussão acima sobre a conjetura de Markus-Yamabe revela como é rica a relação entre a dinâmica de um sistema $x^{\prime}=X(x)$ com certas condições sobre o espectro do campo vetorial $X$ assim como sua injetividade. Podemos citar vários outros trabalhos na mesma linha tais como [15, 21, 22, 24, 25, 30, 31, 32, 33. Em particular, ressaltamos o recente artigo [51], no qual Rabanal estuda os campos planos cujo conjunto de pontos singulares se resume a um centro. Como principal resultado o autor conclui que dado um campo vetorial diferenciável $X: \mathbb{R}^{2} \rightarrow \mathbb{R}^{2}$, se $X(O)=O$ e $\operatorname{Spec}(X) \subset\{z \in \mathbb{C} ; \operatorname{Re}(z)=0\} \backslash\{0\}$, então para todo $p \in \mathbb{R}^{2}$ existe uma única trajetória passando por $p$ e a origem é um centro global, ou seja, para todo $q \in \mathbb{R}^{2} \backslash\{O\}$ a órbita passando por $q$ é periódica.

Visando dar uma continuidade no estudo dos sistemas dinâmicos contínuos bidimensionais com um único ponto singular, na primeira parte deste trabalho propomos um estudo do caso em que tal ponto de equilíbrio é do tipo sela hiperbólica, o qual foi apresentado em [15]. Note que isto completa, genericamente, o estudo dos campos vetoriais planares com um único ponto de equilíbrio.

Denotaremos por $\mathfrak{X}^{1}(U)$ o conjunto dos campos vetoriais $X: U \rightarrow \mathbb{R}^{2}$, de classe $C^{1}$, onde $U$ é um aberto de $R^{2}$. E por $\mathfrak{X}_{s}^{1}\left(\mathbb{R}^{2}\right)$ o subconjunto de $\mathfrak{X}^{1}\left(\mathbb{R}^{2}\right)$ dos campos com um único ponto singular, posicionado na origem $O$, que é do tipo sela hiperbólica.

Sejam $A$ um subconjunto Lebesgue mensurável de $\mathbb{R}^{n}$ e $f: A \rightarrow \mathbb{R}$ uma função mensurável. Considere a seguinte notação:

$$
f^{+}(x)=\max \{f(x), 0\} \text { e } f^{-}(x)=\max \{-f(x), 0\} .
$$

Seguindo [1], uma função $f: A \rightarrow \mathbb{R}$ será dita quase-integrável se

$$
\min \left\{\int_{A} f^{+} d \lambda, \int_{A} f^{-} d \lambda\right\}<\infty
$$

neste caso define-se

$$
\int_{A} f d \lambda=\int_{A} f^{+} d \lambda-\int_{A} f^{-} d \lambda
$$


o qual é um valor bem definido na reta real estendida $[-\infty, \infty]$. Se $X \in \mathfrak{X}^{1}(U)$, escrevemos por $\operatorname{Div}(X): U \rightarrow \mathbb{R}$ a função (contínua) que a cada $p \in U$ associa o valor real $\operatorname{Traço~}\left(D_{p} X\right)$.

Uma das principais ferramentas nos trabalhos [22, 30, 51], onde se obteram condições para que um ponto de equilíbrio fosse atrator global ou centro global, é a não existência de setores pseudo-hiperbólicos no infinito (cf. Definição 2.5). Este também será nosso ponto de partida. Em [1], os autores provaram que $X \in \mathfrak{X}^{1}\left(R^{2}\right)$ não possui setores pseudo-hiperbólicos no $\infty$ sempre que $\operatorname{Div}(X)$ for quase-integrável e, para algum $r>0$,

$$
\int_{r}^{\infty} \inf \{\|X(p)\| ;\|p\|=s\} d s=\infty
$$

Usando estes fatos e considerando a classificação das folheações regulares no plano dadas por Kaplan em [37] e Markus [39], provaremos que:

Teorema A. Se $X \in \mathfrak{X}_{s}^{1}\left(\mathbb{R}^{2}\right)$ satisfaz a igualdade (0.2) e tem $\operatorname{Div}(X)$ quase-integrável, então $X$ é uma sela global, isto é, topologicamente equivalente à sela linear $L(x, y)=$ $(-x, y)$.

Ressaltamos que neste resultado não é necessária a condição de $X$ ser um difeomorfismo local do plano, o que ocorre na conjetura de Markus-Yamabe e no resultado de centro global dado por Rabanal, devido à hipótese espectral. Analogamente a Olech em [46], no caso particular em que $X \in \mathfrak{X}_{s}^{1}\left(\mathbb{R}^{2}\right)$ satisfaz $\operatorname{Spec}(X) \cap\{0\}=\varnothing$, mostraremos no Lema 2.11 que existe uma estreita relação entre a injetividade de $X$ e o fato de tal campo ser uma sela global.

A partir da relação obtida no Lema 2.11, estabelecemos o seguinte resultado análogo ao de Markus-Yamabe:

Teorema B. Seja $X \in \mathfrak{X}^{1}\left(\mathbb{R}^{2}\right)$ um campo vetorial com um ponto singular na origem $O$ de $\mathbb{R}^{2}$. Se $\operatorname{Div}(X)$ é quase-integrável e, para algum $\epsilon>0$,

$$
\operatorname{Spec}(X) \cap[0, \epsilon)=\varnothing \quad \text { ou } \operatorname{Spec}(X) \cap(-\epsilon, 0]=\varnothing,
$$

então X é uma sela global.

Na Seção 2.1.2, as hipóteses deste teorema serão comparadas com as da conjetura de Markus-Yamabe, aqui nos limitaremos a ressaltar que o Exemplo 2.17 ilustra que a hipótese espectral 0.3 não poderia ser relaxada para, por exemplo, $\operatorname{Spec}\left(D X_{q}\right)=$ $\left\{\lambda_{1}(q)<0, \lambda_{2}(q)>0\right\}$, para todo $q \in \mathbb{R}^{2}$, o que seria uma condição análoga à de MarkusYamabe no caso do ponto singular ser uma sela hiperbólica. 
Outra questão tratada no Capítulo 2, especificamente na Seção 2.1.4, é o estudo das folheações com uma única singularidade tipo sela hiperbólica realizadas pelas órbitas de um campo. Dado um campo vetorial $X \in \mathfrak{X}_{s}^{1}\left(\mathbb{R}^{2}\right)$, as órbitas de $X$ induzem uma folheação (com uma única singularidade tipo sela) em $\mathbb{R}^{2}$. Como consequência do Teorema de Poincaré-Bendixon, as quatro separatrizes da sela $O$ de $X$, fornecem de maneira natural uma decomposição do plano em quatro regiões conexas homeomorfas a $\mathbb{R}^{2}$, que denotaremos por $W_{i}, i=0, \ldots, 3$, nas quais as folheações induzidas pelas órbitas de $X$ são regulares. Pela classificação realizada por Markus em [39], um campo $X \in \mathfrak{X}_{s}^{1}\left(\mathbb{R}^{2}\right)$ será uma sela global se, e somente se, as folheações em $W_{i}$ são triviais para todo $i \in\{0, \ldots, 3\}$. Por outro lado, veremos por meio de exemplos que, dado $X \in \mathfrak{X}_{s}^{1}\left(\mathbb{R}^{2}\right)$, nem sempre tais folheações são triviais (cf. Exemplo 2.1). Isto motiva a seguinte questão:

É possível caracterizar as folheações (regulares) nas regiões $W_{i}$ para campos vetoriais em $\mathfrak{X}_{s}^{1}\left(\mathbb{R}^{2}\right)$ ?

O que apresentamos na Seção 2.1.4 é uma resposta incompleta para esta pergunta. Com efeito, damos uma caracterização parcial para tais folheações. O fato das folheações nas regiões $W_{i}$ não possuírem singularidades nos permite lançar mão da classificação das folheações regulares do plano realizadas por Kaplan [37]. Está classificação baseia-se na partição das folheações do plano em regiões canônicas separadas entre si por órbitas especiais chamadas separatrizes. Analogamente ao caso das selas globais, uma das ferramentas fundamentais aqui será o que chamamos, na Definição 2.26, de setor pseudo-hiperbólico inicial no infinito. Com a ajuda da teoria de campos vetoriais em rotação, rapidamente apresentada no início da Seção 2.1.4, dividimos cada região $W_{i}$ em dois domínios. O fato das regiões canônicas, contidas no domínio coberto pelas separatrizes em rotação da sela $O$ de $X$, não possuírem setores pseudo-hiperbólicos iniciais (ver Lema 2.28), nos permite caracterizar as folheações em tais regiões. Tal caracterização é feita por meio de árvores (uma classe de grafos) no Teorema 2.30 .

No tocante aos sistemas dinâmicos discretos, analogamente ao caso contínuo, nosso foco será nos difeomorfismos do plano com um único ponto fixo que será do tipo sela hiperbólica. As questões que iremos abordar estão relacionadas com dois importantes problemas.

I) Seguindo a linha de estabelecer um paralelo entre sistemas contínuos e discretos, muitos autores têm estudado questões sobre a unicidade e a estabilidade de pontos fixos de aplicações do plano em si mesmo. Veja por exemplo [2, 3, 17, 14, 13, 20] e as referências aí contidas. Neste contexto, destacamos a versão discreta da conjetura discutida acima, a qual foi estabelecida em 1995 por Cima, Gasull e Mañosas em [14]: 
Conjetura discreta de Markus-Yamabe: Seja $f: \mathbb{R}^{n} \rightarrow \mathbb{R}^{n}$ uma aplicação de classe $C^{1}$ tal que $f(O)=O$ e para todo $x \in \mathbb{R}^{n}$, os autovalores de $J f(x)$ têm módulo menor que 1. Então o ponto fixo $O$ é um atrator global do sistema dinâmico discreto gerado por $f$ ?

Em [14] os autores apresentam uma aplicação racional $g: \mathbb{R}^{2} \rightarrow \mathbb{R}^{2}$, construída por Szlenk, que dá uma resposta negativa para o caso bidimensional. Porém, $g$ não é polinomial nem difeomorfismo. Assim, no mesmo trabalho os autores apresentam, através de uma modificação do exemplo de Szlenk, um difeomorfismo do plano que é um contraexemplo para a conjetura discreta. No artigo [13] de 1997, os autores apresentaram um contra-exemplo polinomial para $n \geq 3$. Por outro lado, em 2008 Alarcón, Guíñez e Gutierrez consideram em [2] condições adicionais sob as quais um mergulho de $\mathbb{R}^{2}$ em si mesmo tivesse um único ponto fixo, sendo este um atrator global. Em particular, eles requerem a existência de uma curva não limitada invariante em relação ao mergulho com um extremo em $O$.

Assim como no caso contínuo, no presente trabalho propomos o estudo do caso hiperbólico complementar da conjetura discreta de Markus-Yamabe, isto é, estudar difeomorfismos com um único ponto fixo hiperbólico que é do tipo sela hiperbólica. Na Seção 2.1 tal estudo é realizado para os campos vetoriais. Entre outros resultados, encontramos condições assegurando que tal campo é topologicamente equivalente a uma sela linear. Na Seção 2.2 lidamos com difeomorfismos mais gerais que aqueles induzidos das aplicações associadas a fluxos tomando o tempo unitário.

II) Por outro lado, ao trabalhar em sistemas discretos, existe uma considerável diferença entre o caso de atrator global e o caso sela, devida a existência de variedades estável e instável de dimensão 1 somente no último caso. Com efeito, se a variedade estável do ponto sela intersecta transversalmente sua variedade instável segue, por um resultado clássico, que o difeomorfismo tem um shift de Bernoulli como um subsistema. Nossas hipóteses são em certo sentido complementares. Sempre que necessário, em nossos resultados assumiremos que tais interseções não existem e que uma das separatrizes se acumula somente no infinito. Com tais hipóteses, nosso trabalho passa a ter uma relação mais estreita com o caso de difeomorfismos que têm um atrator global.

Para fixar a notação, considere um difeomorfismo $f: \mathbb{R}^{2} \rightarrow \mathbb{R}^{2}$, de classe $C^{1}$, com um único ponto fixo na origem $O$ de $\mathbb{R}^{2}$, o qual é do tipo sela hiperbólica. Vamos denotar por $\operatorname{Diff}_{s}\left(\mathbb{R}^{2}\right)$ o conjunto de tais difeomorfismos e por $\operatorname{Diff} f_{s}^{+}\left(\mathbb{R}^{2}\right)$ a interseção de $\operatorname{Diff}\left(\mathbb{R}^{2}\right)$ com o conjunto de difeomorfismos do plano que preservam orientação. Dizemos que um difeomorfismo $f$ do plano está no conjunto $\mathcal{S} \subset \operatorname{Diff}_{s}\left(\mathbb{R}^{2}\right)$ se para todo ponto $p \in \mathbb{R}^{2}$, exceto por $O$ e suas separatrizes, temos $\omega(p)=\alpha(p)=\varnothing$ e as quatro separatrizes de $O$ se acumulam só no infinito. Os principais resultados que serão apresentados no âmbito 
discreto são:

Teorema C. Seja $f \in D i f f_{s}^{+}\left(\mathbb{R}^{2}\right)$ tal que $O$ possui uma separatriz $f$-invariante se acumulando somente no infinito $e s_{i} \cap s_{j}=\varnothing$ ou $s_{i}=s_{j}$, para $i \neq j \in\{0, \ldots, 3\}$. Então $f \in \mathcal{S}$.

O próximo resultado trata de difeomorfismos que não necessariamente preservam orientação.

Teorema D. Se $f \in \operatorname{Diff}_{s}\left(\mathbb{R}^{2}\right)$ possui uma separatriz acumulando somente no infinito $e$ não possui órbitas 2 -periódicas, então $f \in \mathcal{S}$.

Como um passo prévio ao estudo dos sistemas dinâmicos contínuos no caso $n$-dimensional para $n \geq 3$, dedicamos o Capítulo 3 ao estudo da injetividade de difeomorfismos locais entre $\mathbb{R}^{n}$. Vale lembrar, que tal assunto possui em si mesmo grande relevância, o que se mostra pela enorme quantidade de trabalhos publicados nas últimas décadas, nos quais o tema tem sido atacado por várias áreas da matemática, tais como: álgebra, geometria algébrica, análise, topologia, etc.

Neste trabalho, dentre as ferramentas que serão utilizadas para atacar o problema de injetividade, se destaca a teoria de folheações. Nosso principal resultado é uma versão fraca da Conjectura Jacobiana real de Jelonek para aplicações polinomiais do $\mathbb{R}^{n}$. A seguir introduzimos alguns conceitos necessários para enunciar tal resultado.

Dada uma aplicação contínua $X: M \rightarrow N$ entre espaços localmente compactos, dizemos que $X$ é não própria em um ponto $p \in N$, se não existir vizinhança $U$ do ponto $p$ tal que a pré-imagem $X^{-1}(\bar{U})$ seja compacta. Denotamos por $S_{X}$ o conjunto dos pontos nos quais a aplicação $X$ não é própria.

No âmbito das aplicações polinomiais, em 2002 Jelonek provou em [36] que: se $X$ : $\mathbb{R}^{n} \rightarrow \mathbb{R}^{n}, n \geq 3$, é um difeomorfismo local polinomial e $\operatorname{codim}\left(S_{X}\right) \geq 3$, então $X$ é um difeomorfismo global. Por outro lado, no exemplo de Pinchuck, dado em [49], que é um caso de difeomorfismo local polinomial do plano não injetivo, temos que $\operatorname{codim}\left(S_{X}\right)=1$ (cf. [34]). Estes fatos motivaram Jelonek a estabelecer em a seguinte:

Conjetura Jacobiana real de Jelonek: Seja $X: \mathbb{R}^{n} \rightarrow \mathbb{R}^{n}$ uma aplicação polinomial tal que $\operatorname{Spec}(X) \cap\{0\}=\varnothing$. Se $\operatorname{codim}\left(S_{X}\right) \geq 2$ então $X$ é bijetiva.

Pelo que vimos acima, o único caso de interesse é quando $\operatorname{codim}\left(S_{X}\right)=2$. Ainda em [36], Jelonek provou que sua conjetura é verdadeira no caso bidimensional. Além disto, em 2009 Gutierrez e Maquera provaram em [28] uma versão fraca no caso tridimensional, considerando adicionalmente a hipótese spectral: $\operatorname{Spec}(Y) \cap[0, \epsilon)=\varnothing$, para algum $\epsilon>0$. 
Outro fato, provado por Jelonek em [36], que ilustra a relevância de sua conjetura é que uma resposta afirmativa para ela implica na solução da famosa conjetura Jacobiana enunciada pela primeira vez em [38] por Keller em 1939, e é o décimo sexto problema da lista elaborada em 1998, por Smale [56], dos dezoito problemas matemáticos mais importantes ainda sem solução:

Conjetura Jacobiana: Seja $X: \mathbb{C}^{n} \rightarrow \mathbb{C}^{n}$ uma aplicação polinomial com Jacobiano não nulo em todo ponto, então $X$ é um isomorfismo, isto é, invertível com inversa polinomial.

Dado um $C^{2}$-difeomorfismo local $X=\left(f_{1}, \ldots, f_{n}\right): \mathbb{R}^{n} \rightarrow \mathbb{R}^{n}$, cada submersão $f_{i}: \mathbb{R}^{n} \rightarrow \mathbb{R}$ induz uma $C^{2}$-folheação $\mathcal{F}_{i}$ de codimensão 1 em $\mathbb{R}^{n}$, cujas folhas são as componentes conexas das hipersuperfícies de nível $f_{i}=$ cte. Como um passo prévio para obter seu resultado tridimensional de bijetividade, Gutierrez e Maquera provaram em [28] o seguinte resultado:

Proposição 0.1. Dada $X=\left(f_{1}, f_{2}, f_{3}\right): \mathbb{R}^{3} \rightarrow \mathbb{R}^{3}$ uma aplicação $C^{2}$ satisfazendo $\operatorname{Spec}(X) \cap(-\epsilon, \epsilon)=\varnothing$, para algum $\epsilon>0$. Então as folheações $\mathcal{F}_{i}$ são por planos, isto é, toda folha é homeomorfa a $\mathbb{R}^{2}$, para todo $i=1,2,3$.

Seja $X=\left(f_{1}, \ldots, f_{n}\right): \mathbb{R}^{n} \rightarrow \mathbb{R}^{n}$ um difeomorfismo local, de classe $C^{2}$. Dada uma $k$ combinação $\left\{i_{1}, \ldots, i_{k}\right\}$ do conjunto $\{1, \ldots, n\}$, as folheações $\mathcal{F}_{i_{1}}, \ldots, \mathcal{F}_{i_{k}}$ são transversais entre si e a interseção $\mathcal{F}_{i_{1} \ldots i_{k}}=\mathcal{F}_{i_{1}} \cap \ldots \cap \mathcal{F}_{i_{k}}$ é uma $C^{2}$-folheação de codimensão $k$ em $\mathbb{R}^{n}$.

O principal resultado do Capítulo 3 é a seguinte versão fraca da Conjetura Jacobiana Real de Jelonek no caso $n$-dimensional:

Teorema E. Seja $X=\left(f_{1}, \ldots, f_{n}\right): \mathbb{R}^{n} \rightarrow \mathbb{R}^{n}$ um difeomorfismo local polinomial tal que para cada $(n-2)$-combinação $\left\{i_{1}, \ldots, i_{n-2}\right\}$ de $\{1, \ldots, n\}$, toda folha de $\mathcal{F}_{i_{1} \ldots i_{n-2}}$ é simplesmente conexa. Se $\operatorname{codim}\left(S_{X}\right) \geq 2$ então $X$ é um difeomorfismo global.

Observe que a hipótese topológica que impomos sobre as folhas de $\mathcal{F}_{i_{1} \ldots i_{n-2}}$, é obtida no caso de Gutierrez e Maquera como consequência da hipótese spectral via Proposição 0.1 .

Provaremos também uma versão fraca no caso tridimensional da seguinte conjetura apresentada em [45]:

Conjetura de Nollet e Xavier: Um difeomorfismo local $X: \mathbb{R}^{n} \rightarrow \mathbb{R}^{n}$ é injetivo se a pré-imagem de qualquer hiperplano afim é conexa (possivelmente vazia). 


\section{Preliminares}

Neste capítulo serão introduzidos conceitos e resultados de caráter geral que se utilizarão no decorrer deste trabalho.

\subsection{Campos vetoriais}

Um campo vetorial $X$ de classe $C^{1}$ num subconjunto $U$ aberto do $\mathbb{R}^{n}$, é uma aplicação de classe $C^{1}$ que a cada ponto $p$ de $U$ associa um vetor tangente $X(p)$ em $\mathbb{R}^{n}$. Ao campo vetorial $X$ está associada a equação diferencial

$$
x^{\prime}=X(x)
$$

e vice-versa. Denotaremos por $\mathfrak{X}^{1}(U)$ o conjunto de campos vetoriais $X: U \subset \mathbb{R}^{n} \rightarrow \mathbb{R}^{n}$, de classe $C^{1}$.

Seja $I$ um intervalo aberto da reta $\mathbb{R}$ contendo o zero. Dizemos que uma função $\gamma$ : $I \rightarrow U$, de classe $C^{1}$, é uma solução ou trajetória do campo vetorial $X$ se $\gamma^{\prime}(t)=X(\gamma(t))$, para todo $t \in I$. Para simplificar a notação, identificamos a solução $\gamma$ com sua imagem orientada, a qual denominamos órbita de $X$, definida em $I(\gamma)$. Uma função $\gamma: I \rightarrow U$ chama-se solução máxima se para toda trajetória $\eta: J \rightarrow U$ tal que $I \subseteq J$ e $\gamma=\left.\eta\right|_{I}$ então $J=I$ e consequentemente $\gamma=\eta$. Neste caso $I$ chama-se intervalo maximal. $\mathrm{O}$ conjunto aberto $U$, munido da decomposição em órbitas (imagens orientadas das soluções maximais) de $X$ é chamado retrato de fase de $X$.

O seguinte teorema da teoria clássica de equações diferencias estabelece, além da existência e unicidade de soluções da equação (1.1), a existência de um fluxo de classe $C^{1}$ associado ao campo $X \in \mathfrak{X}^{1}(U)$. Sua demonstração é encontrada em livros que trata tal teoria, como por exemplo [35, 42, 54, 58]. 
Teorema 1.1. Seja $X \in \mathfrak{X}^{1}(U)$.

1) Para cada $p \in U$ existe um intervalo maximal $I\left(\gamma_{p}\right)$ onde está definida a única solução $\gamma_{p}$ da equação (1.1) tal que $\gamma_{p}(0)=p$.

2) Se $q=\gamma_{p}(t)$ para algum $t \in I\left(\gamma_{p}\right)$, então $I\left(\gamma_{q}\right)=I\left(\gamma_{p}\right)-t=\left\{r-t ; r \in I\left(\gamma_{p}\right)\right\}$ e $\gamma_{q}(s)=\gamma_{p}(t+s)$ para todo $s \in I\left(\gamma_{q}\right)$.

3) O conjunto $\Delta=\left\{(t, p) ; p \in U, t \in I\left(\gamma_{p}\right)\right\}$ é aberto em $\mathbb{R}^{n+1}$, a aplicação $\phi: \Delta \rightarrow U$ dada por $\phi(t, p)=\gamma_{p}(t)$ é de classe $C^{1}$ e satisfaz

$$
D_{1} D_{2} \phi(t, p)=D X(\phi(t, p)) \cdot D_{2} \phi(t, p)
$$

para todo $(t, p) \in \Delta$.

A aplicação $\phi: \Delta \rightarrow U$ é chamada fluxo gerado por $X$.

Dizemos que $p \in U$ é um ponto singular ou ponto de equilíbrio de $X$ se $X(p)=O$, caso contrário dizemos que $p$ é um ponto regular de $X$. Uma trajetória $\gamma$ é dita periódica se está definida em $\mathbb{R}$ e existe um real $\tau>0$ tal que $\gamma(t+\tau)=\gamma(t)$ para todo $t \in \mathbb{R}$.

Um ponto singular $p$ de um campo vetorial $X \in \mathfrak{X}^{1}(U)$ é hiperbólico se os autovalores (complexos) da derivada $D X_{p}$ têm parte real não nula. Neste caso, o número de autovalores de $D X_{p}$ que têm parte real negativa será chamado de índice de estabilidade de $X$ em $p$. Um ponto singular $p$ é do tipo sela hiperbólica se seu índice de estabilidade é maior do que zero e menor que $n$.

Dados dois campos vetoriais $X_{1}$ e $X_{2}$ definidos nos abertos $U_{1}$ e $U_{2}$. Dizemos que $X_{1}$ é topologicamente equivalente a $X_{2}$ se existir um homeomorfismo $h: U_{1} \rightarrow U_{2}$ que leva órbita de $X_{1}$ em órbita de $X_{2}$ preservando orientação. Mais precisamente, sejam $p \in U_{1}$ e $\gamma_{1}(p)$ a órbita orientada de $X_{1}$ passando por $p$, então $h\left(\gamma_{1}(p)\right)$ é a órbita orientada $\gamma_{2}(h(p))$ de $X_{2}$ passando por $h(p)$. Tal noção estabelece uma relação de equivalência entre campos vetoriais definidos em abertos de $\mathbb{R}^{n}$.

O próximo resultado estabelece a equivalência topológica local entre dois campos em vizinhanças de pontos singulares hiperbólicos com o mesmo índice de estabilidade (cf. [35, 42, 54, 58]):

Teorema 1.2 (Hartman-Grobman). Seja $U$ um aberto de $\mathbb{R}^{n}$, dado um campo vetorial $X \in \mathfrak{X}^{1}(U)$ com um ponto singular hiperbólico $p$. Existem vizinhanças $V$ de $p$ e $W$ de $O$ em $\mathbb{R}^{n}$ tais que $\left.X\right|_{V}$ é topologicamente equivalente a $\left.D X_{p}\right|_{W}$. 
Seja $\gamma_{p}: I_{p} \rightarrow U$ a solução máxima de $X \in \mathfrak{X}^{1}(U)$ passando por $p$ no instante $0 \in I_{p}$, definida em seu intervalo máximo $I_{p}=\left(\tau_{-}(p), \tau_{+}(p)\right)$. Se $\tau_{+}(p)=\infty\left(\operatorname{resp} \cdot \tau_{-}(p)=-\infty\right)$ define-se o conjunto $\omega(p)=\left\{q \in U ; \exists\left(t_{k}\right)\right.$ com $t_{k} \rightarrow \infty$ e $\gamma_{p}\left(t_{k}\right) \rightarrow q$ quando $\left.k \rightarrow \infty\right\}$ (resp. $\alpha(p)=\left\{q \in U ; \exists\left(t_{k}\right)\right.$ com $t_{k} \rightarrow-\infty$ e $\gamma_{p}\left(t_{k}\right) \rightarrow q$ quando $\left.k \rightarrow \infty\right\}$ ). Os conjuntos $\omega(p)$ e $\alpha(p)$ são chamados respectivamente de conjunto $\omega$-limite e conjunto $\alpha$-limite de $p$. Além disto, introduzimos os seguintes conjuntos limites apresentados por Markus em [39]: $\gamma_{p+}=\overline{\gamma_{p}(t \geq 0)} \backslash \gamma_{p}(t \geq 0)$ e $\gamma_{p-}=\overline{\gamma_{p}(t \leq 0)} \backslash \gamma_{p}(t \leq 0)$. Estes conjuntos serão úteis na construção dos conceitos de separatrizes e regiões canônicas de campos. Observe que na definição de $\gamma_{p+}$ e $\gamma_{p-}$ não é necessário que $\tau_{+}=\infty$ nem $\tau_{-}=-\infty$.

Nosso próximo passo será enunciar o Teorema das variedades estável e instável para pontos de equilíbrio de um campo. Antes porém introduziremos alguns conceitos. Sejam $X \in \mathfrak{X}^{r}(U)$ um campo vetorial e $p \in U$ um ponto singular hiperbólico. Neste caso, é bem conhecido que existe uma decomposição em soma direta $\mathbb{R}^{n}=E^{s} \oplus E^{u}$, onde $E^{s}$ e $E^{u}$ são os subespaços estável e instável, respectivamente, associados ao operador linear $D X_{p}: \mathbb{R}^{n} \rightarrow \mathbb{R}^{n}$. Isto é, $E^{s}$ (resp. $E^{u}$ ) é invariante por $D X_{p}$ e a restrição $\left.D X_{p}\right|_{E^{s}}$ (resp. $\left.D X_{p}\right|_{E^{u}}$ ) tem todos seus autovalores com parte real negativa (resp. positiva). É claro que a dimensão do subespaço estável $E^{s}$ coincide com o índice de estabilidade do ponto $p$. Dado $\epsilon>0$, denotamos por $E^{j}(\epsilon)$ o subconjunto $\left\{v \in E^{j} ;\|v\|<\epsilon\right\}$, para $j=s, u$. Dada uma vizinhança $V \subset U$ de $p$, definimos a variedade estável local (resp. variedade instável local) de $p$ por $W_{p}^{s}(V)=\{q \in V ; \phi(t, q) \rightarrow p$, quando $t \rightarrow \infty\}$ (resp. $W_{p}^{u}(V)=\{q \in V ; \phi(t, q) \rightarrow p$, quando $\left.t \rightarrow-\infty\}\right)$, onde $\phi$ é o fluxo associado a $X$.

Teorema 1.3 (Teorema das variedades estável e instável). Seja $p$ um ponto singular hiperbólico de um campo vetorial $X \in \mathfrak{X}^{r}(U)$, cujo indice de estabilidade é d. Existe uma vizinhança $V$ de $p$ tal que $W_{p}^{s}(V)$ e $W_{p}^{u}(V)$ são discos $C^{r}$ mergulhados, tangentes $a E^{s}$ e $E^{u}$ no ponto $p$, de dimensões $d$ e $n-d$, respectivamente. De fato, considerando $\mathbb{R}^{n}=E^{s} \times E^{u}$, existe $\epsilon$, suficientemente pequeno, tal que tomando $V=p+E^{s}(\epsilon) \times E^{u}(\epsilon)$, $W_{p}^{u}(V)$ é o gráfico de uma função $\sigma^{u}: E^{u}(\epsilon) \rightarrow E^{s}(\epsilon)$, de classe $C^{r}$, com $\sigma^{u}(0)=0$ e $D \sigma_{0}^{u}=0$. Ou seja,

$$
W_{p}^{u}(V)=\left\{p+\left(x, \sigma^{u}(x)\right) ; x \in E^{s}(\epsilon)\right\} .
$$

Analogamente, existe uma função $\sigma^{s}: E^{s}(\epsilon) \rightarrow E^{u}(\epsilon)$, de classe $C^{r}$, com $\sigma^{s}(0)=0$ e $D \sigma_{0}^{s}=0$ tal que

$$
W_{p}^{s}(V)=\left\{p+\left(\sigma^{s}(y), y\right) ; y \in E^{u}(\epsilon)\right\}
$$

\subsubsection{Campos vetoriais planares}

Iremos agora introduzir alguns resultados válidos para o caso particular em que $U$ é um aberto de $\mathbb{R}^{2}$. Começamos por uma importante ferramenta para o estudo qualitativo 
dos sistemas dinâmicos contínuos no plano:

Teorema 1.4 (Poincaré-Bendixon). Sejam $X \in \mathfrak{X}^{1}(U)$ e $\phi$ seu fluxo associado. Dada uma trajetória $\gamma(t)=\phi(t, p)$ de $X$ passando por $p$, tal que $\gamma(t)$ está contida num compacto $K \subset U$, para todo $t \geq 0$. Se $X$ possui um número finito de singularidades em $\omega(p)$, então têm-se as seguintes alternativas:

1) Se $\omega(p)$ contém somente pontos regulares, então $\omega(p)$ é uma órbita periódica.

2) Se $\omega(p)$ contém pontos regulares e singulares, então $\omega(p)$ consiste de um conjunto de órbitas, cada uma das quais tende a um dos pontos singulares quando $t \rightarrow \pm \infty$.

3) Se $\omega(p)$ não contém pontos regulares, então $\omega(p)$ é um ponto singular.

Dado um campo vetorial $X \in \mathfrak{X}^{1}(U)$, um ponto singular $p$ de $X$ é uma sela hiperbólica se, e somente se, o índice de estabilidade de $X$ em $p$ é igual a 1 , ou equivalentemente, os autovalores $\lambda_{1}$ e $\lambda_{2}$ da derivada $D X_{p}$ satisfazem $\lambda_{1} \lambda_{2}<0$. Se $p$ é uma sela hiperbólica de $X$, definimos por separatriz estável (resp. instável) de $p$ uma solução $\gamma$ de $X$ satisfazendo $\omega(\gamma)=p(\operatorname{resp} . \alpha(\gamma)=p)$

Se $U \subset \mathbb{R}^{2}$ é simplesmente conexo, então segue da teoria do índice que toda órbita periódica de $X$ borda uma região compacta contendo um ponto singular de $X$. Dizemos que uma órbita periódica $\Gamma$ é um ciclo limite de $X$ se existe uma órbita $\gamma$ de $X$ tal que $\gamma \cap \Gamma=\varnothing$ e $\omega(\gamma)=\Gamma$ ou $\alpha(\gamma)=\Gamma$.

Seja $X \in \mathfrak{X}^{1}\left(\mathbb{R}^{2}\right)$ um campo vetorial. Uma região planar $V$ de $\mathbb{R}^{2}$ (subconjunto aberto e conexo de $\mathbb{R}^{2}$ ) é dita paralela se for constituída por órbitas de $X$ e a restrição $\left.X\right|_{V}$ for topologicamente equivalente a um dos seguintes campos vetoriais

a) $X: \mathbb{R}^{2} \rightarrow \mathbb{R}^{2}$ dado por $X(x, y)=(1,0)$ (tipo faixa);

b) $X: \mathbb{R}^{2} \backslash\{O\} \rightarrow \mathbb{R}^{2}$ definido em coordenadas polares por $X(r, \theta)=(0,1)$ (tipo anelar);

c) $X: \mathbb{R}^{2} \backslash\{O\} \rightarrow \mathbb{R}^{2}$ definido em coordenadas polares por $X(r, \theta)=(1,0)$ (tipo radial).

Definição 1.5. Dado um campo vetorial $X \in \mathfrak{X}^{1}\left(\mathbb{R}^{2}\right)$, segundo Markus em [39], dizemos que uma órbita $\gamma$ é uma separatriz de $X$ se não existir uma vizinhança paralela $V$ de $\gamma$ satisfazendo as seguintes propriedades:

a) toda solução de $X$ em $V$ têm os mesmos conjuntos limites $\gamma_{-} e \gamma_{+}$; 
b) a fronteira de $V$ é dada por $\gamma_{-} \cup \gamma_{+}$e exatamente duas soluções $\gamma^{1}$ e $\gamma^{2}$ para as quais $\gamma_{-}^{1}=\gamma_{-}^{2}=\gamma_{-} e \gamma_{+}^{1}=\gamma_{+}^{2}=\gamma_{+}$.

Exemplo 1.6. Dado $X \in \mathfrak{X}^{1}\left(\mathbb{R}^{2}\right)$, segue da definição de separatrizes que todo ponto singular de $X$ é uma separatriz de $X$. Uma órbita fechada é uma separatriz de $X$ se, e somente se, for um ciclo limite. Se p é um ponto singular de $X$ do tipo sela hiperbólica, então cada separatriz de $p$ é também uma separatriz de $X$. Ou seja, o conceito de separatriz (estável e/ou instável) de um ponto singular do tipo sela hiperbólica é um caso particular de separatriz do campo $X$.

Seja $\zeta$ o conjunto de todas as separatrizes de um campo vetorial $X \in \mathfrak{X}^{1}\left(\mathbb{R}^{2}\right)$. Se uma separatriz $\gamma$ está contida em $\overline{\zeta \backslash \gamma}$, então $\gamma$ é uma separatriz limite de $X$. Em [39], Markus prova que $\zeta$ é um subconjunto fechado de $R^{2}$. Cada componente conexa do aberto $\mathbb{R}^{2} \backslash \zeta$ é chamada de região canônica de $X$. Observe que se duas órbitas $\gamma^{1}$ e $\gamma^{2}$ de $X$ pertencem a uma mesma região canônica de $X$ então $\gamma_{-}^{1}=\gamma_{-}^{2}$ e $\gamma_{+}^{1}=\gamma_{+}^{2}$. Além disto, $\gamma^{1}$ é uma órbita fechada se, e somente se, $\gamma^{2}$ também o é. Ainda em [39], o autor mostra o seguinte resultado sobre a natureza das regiões canônicas:

Proposição 1.7. Toda região canônica de um campo vetorial $X \in \mathfrak{X}^{1}(\mathbb{R})^{2}$ é paralela, isto é, topologicamente equivalente ao campo tipo faixa, anelar ou radial.

Seja $X \in \mathfrak{X}^{1}\left(\mathbb{R}^{2}\right)$ um campo vetorial. Chamamos de configuração de separatrizes de $X$, e denotamos por $C \zeta$, ao conjunto consistindo de todas as separatrizes de $X$ e de uma solução de cada região canônica de $X$. O seguinte resultado, dado em [39], diz que a configuração de separatrizes é um invariante num subconjunto de $\mathfrak{X}^{1}(\mathbb{R})^{2}$ :

Teorema 1.8. Sejam $X_{1}, X_{2} \in \mathfrak{X}^{1}\left(\mathbb{R}^{2}\right)$ campos vetoriais sem separatrizes limites distintas de pontos singulares e $C \zeta_{1}, C \zeta_{2}$ suas respectivas configurações de separatrizes. Então $X_{1}$ é topologicamente equivalente a $X_{2}$ se, e somente se, existe um homeomorfismo do plano em si mesmo que leva órbitas de $C \zeta_{1}$ em órbitas de $S \zeta_{2}$ preservando orientação.

\subsection{Folheações}

Nesta seção introduzimos o conceito de folheações regulares em variedades diferenciáveis e enunciamos alguns resultados básicos relacionados a estes objetos. Neste texto, será comum o uso do termo folheação no lugar de folheação regular e será explicitado quando folheação possuir singularidades. Para uma leitura mais completa sobre o assunto indicamos [10, 11, 12, 44]. 
Definição 1.9. Uma folheação regular de classe $C^{r}$ e de dimensão $k$ (ou codimensão $n=m-k$ ) de uma variedade $M^{m}$ é um atlas máximo $\mathcal{F}$ de classe $C^{r}$ de $M$ tal que:

1) se $(U, \varphi) \in \mathcal{F}$, então $\varphi(U)=U_{1} \times U_{2}$ onde $U_{1}$ e $U_{2}$ são discos abertos de $\mathbb{R}^{k}$ e $\mathbb{R}^{m-k}$, respectivamente;

2) se $(U, \varphi),(V, \psi) \in \mathcal{F}$ são tais que $U \cap V \neq \varnothing$, então a mudança de coordenadas $\varphi \circ \psi^{-1}: \psi(U \cap V) \rightarrow \varphi(U \cap V)$ é de classe $C^{r}$ e tem a forma

$$
\varphi \circ \psi^{-1}(x, y)=(f(x, y), g(y))
$$

Dada uma carta local $(U, \varphi) \in \mathcal{F}$ tal que $\varphi(U)=U_{1} \cap U_{2}$, as placas de $\mathcal{F}$ são os conjuntos da forma $\varphi^{-1}\left(U_{1} \times\{c\}\right), c \in U_{2}$, as quais são subvariedades conexas de dimensão $k$ de classe $C^{r}$ de $M$. Um caminho de placas de $\mathcal{F}$ é uma sequência $\alpha_{1}, \ldots, \alpha_{s}$ de placas de $\mathcal{F}$ tal que $\alpha_{j} \cap \alpha_{j+1} \neq \varnothing$ para todo $j \in\{1, \ldots, s-1\}$. As folhas de $\mathcal{F}$ são as classes da relação de equivalência definida sobre $M$ da seguinte maneira: $p \sim q$ se existe um caminho de placas $\alpha_{1}, \ldots, \alpha_{s}$ tal que $p \in \alpha_{1}$ e $q \in \alpha_{s}$. Toda folha $L$ de $\mathcal{F}$ possui uma estrutura de variedade $C^{r}$ naturalmente induzida pelas cartas de $\mathcal{F}$ (veja [10]).

O próximo resultado é uma consequência da Forma Local das Submersões.

Proposição 1.10. Toda submersão $f: M^{m} \rightarrow N^{n}$, de classe $C^{r}$, entre as variedades $M$ e $N$, induz uma $C^{r}$-folheação de codimensão $n$ em $M$, onde as folhas são as superfícies de nivel da submersão $f$.

Definição 1.11. Seja $M^{m}$ uma variedade folheada por uma folheação $\mathcal{F}$ de dimensão $k<m$. O espaço das folhas de $\mathcal{F}$, denotado por $M / \mathcal{F}$, é o espaço quociente de $M$ pela relação de equivalência $\sim$ que identifica dois pontos de $M$ se eles estão na mesma folha de $\mathcal{F}$. Em $M / \mathcal{F}$ consideramos a topologia quociente $\tau_{q}$.

A noção de equivalência entre folheações é dada por:

Definição 1.12. Sejam $\left(M_{1}, \mathcal{F}_{1}\right)$ e $\left(M_{2}, \mathcal{F}_{2}\right)$ variedades folheadas. Dizemos $\left(M_{1}, \mathcal{F}_{1}\right)$ é topologicamente equivalente a $\left(M_{2}, \mathcal{F}_{2}\right)$ se existe um homeomorfismo $H: M_{1} \rightarrow M_{2}$ que leva folha em folha.

Dada uma folheação $\mathcal{F}$ de codimensão $n$ e de classe $C^{r}$ de $M$, relembramos, a seguir, a noção de holonomia de uma folha $L$ de $\mathcal{F}$.

Sejam $\gamma:[0,1] \rightarrow L$ um caminho contínuo tal que $\gamma(0)=\gamma(1)=p$ e $\Sigma$ uma seção transversal a $L$ em $p$. Consideremos uma sequência de cartas locais $\left(U_{i}\right)_{i=0}^{s}$ e uma partição 
$0=t_{0}<\ldots<s+1=1$ do intervalo $[0,1]$ tais que, se $U_{i} \cap U_{j} \neq \varnothing$ então $U_{i} \cup U_{j}$ está contido numa carta local de $\mathcal{F}$ e $\gamma\left(\left[t_{i}, t_{i+1}\right]\right) \subset U_{i}$ para todo $0 \leq i \leq s$. Fixando seções transversais a $\mathcal{F}, D\left(t_{i}\right) \subset U_{i-1} \cap U_{i}$, homeomorfos a um disco de dimensão $n$ passando por $\gamma\left(t_{i}\right)$ para cada $0 \leq i \leq s+1$ com $D\left(t_{0}\right)=D\left(t_{s+1}\right)=\Sigma$, então para cada $x \in D\left(t_{i}\right)$ suficientemente próximo de $\gamma\left(t_{i}\right)$, a placa de $U_{i}$ que passa por $x$ intercepta $D\left(t_{i+1}\right)$ num único ponto $f_{i}(x)$. A aplicação de holonomia associada a $\gamma$ é dada pela composição

$$
f_{\gamma}=f_{s} \circ f_{s-1} \circ \ldots f_{0}
$$

e não depende dos discos $D\left(t_{i}\right), i=0,1, \ldots, s$, nem da sequência de cartas locais $\left(U_{i}\right)_{i=0}^{s}$.

Sejam $G(\Sigma, p)$ o grupo de germes de difeomorfismos $C^{r}$ de $\Sigma$ que deixam fixo $p$, e $\varphi_{\gamma}$ o germe da aplicação de holonomia $f_{\gamma}$ em $p$. Então a transformação $\gamma \rightarrow \varphi_{\gamma^{-1}}$ induz um difeomorfismo

$$
\Phi: \pi_{1}(L, p) \rightarrow G(\Sigma, p)
$$

dado por $\Phi([\gamma])=\varphi_{\gamma^{-1}}$. O grupo de holonomia de $L$ em $p$ é o subgrupo de $G(\Sigma, p)$ definido por $\operatorname{Hol}(L, p)=\Phi\left(\pi_{1}(F, p)\right)$, o qual não depende do ponto $p$, o que nos permite falar simplesmente no grupo de holonomia da folha $L$.

Observação 1.13. Não é difícil ver que a holonomia de uma folheação induzida por submersão é trivial, isto é, $\operatorname{Hol}(L, p)=\{i d:(\Sigma, p) \rightarrow(\Sigma, p)\}$.

Definição 1.14. Seja $\mathcal{F}$ uma folheação em $M$, diremos que um subconjunto $A \subset M$ é saturado por $\mathcal{F}$, ou é $\mathcal{F}$-invariante, se $A$ é uma união de folhas de $\mathcal{F}$.

A seguir enunciamos o Teorema da estabilidade local de Reeb que foi provado em [52, 53] e será aplicado no Capítulo 3 .

Teorema 1.15. Seja $\mathcal{F}$ uma folheação de classe $C^{1}$ e codimensão $n$ de uma variedade diferenciável $M$ e $L \in \mathcal{F}$ uma folha compacta com grupo de holonomia finito. Existe uma vizinhança $U$ de $L$, dada por uma união de folhas de $F$, na qual todas as folhas são compactas com grupo de holonomia finito.

Definição 1.16. Seja $M$ uma variedade com uma folheação $\mathcal{F}$. Um subconjunto $\mu \subset M$ é minimal se satisfazer as seguintes propriedades:

- $\mu$ é compacto, não vazio e invariante,

- se $\mu^{\prime} \subset \mu$ é um subconjunto fechado e invariante então $\mu^{\prime}=\varnothing$ ou $\mu^{\prime}=\mu$.

Extraímos de [10] as seguintes propriedades sobre conjuntos minimais: 
Teorema 1.17. 1) Toda folha compacta de uma folheação é um conjunto minimal.

2) Toda folheação em uma variedade compacta tem conjunto minimal.

3) Toda folha contida num conjunto minimal é densa neste conjunto.

Aplicaremos também no Capítulo 3 o seguinte resultado devido a Sacksteder [55] o qual relaciona a existência de conjuntos minimais, distintos de folhas compactas, com a não trivialidade do grupo de holonomia da folheação.

Teorema 1.18. Seja $M$ uma variedade e suponha que $\mu \subset M$ é um conjunto minimal de uma folheação $\mathcal{F}$ de classe $C^{2}$ e codimensão 1 que não é uma folha compacta nem toda a variedade. Então existe uma folha $L \in \mathcal{F}$ com holonomia e grupo fundamental não triviais.

A seguir introduzimos a noção de posição geral de uma aplicação em relação a uma folheação. Nosso principal objetivo é enunciar o Teorema 1.21, o qual será aplicado no Capítulo 3. Salientamos que os conceitos assim como os resultados são válidos para dimensão finita qualquer, porém nos restringiremos ao caso que utilizaremos, a saber o bidimensional.

Seja $D^{2}=\left\{\left(x_{1}, x_{2}\right) \in \mathbb{R}^{2} ; x_{1}^{2}+x_{2}^{2} \leq 1\right\}$ o disco fechado bidimensional com fronteira $\partial D^{2}$. Um ponto $p \in D^{2}$ é um ponto crítico de uma função $f: D^{2} \rightarrow \mathbb{R}$, de classe $C^{2}$, se a derivada $f^{\prime}(p)=0$. Dizemos que um ponto crítico $p$ é não degenerado se sua derivada segunda $f^{\prime \prime}(p)$ é uma forma quadrática simétrica não degenerada, isto é, a matriz Hessiana associada a $f$ no ponto $p$ tem posto 2 , onde

$$
f^{\prime \prime}(p)=\left(\frac{\partial^{2} f}{\partial x_{i} \partial x_{j}}(0)\right)_{1 \leq i, j \leq 2} .
$$

Uma aplicação $f$ do disco $D^{2}$ em $\mathbb{R}$ é uma aplicação de Morse se for uma função de classe $C^{2}$ cujos pontos críticos são todos não degenerados. Desde que $D^{2}$ é compacto, uma função de Morse $f: D^{2} \rightarrow \mathbb{R}$ possui uma quantidade finita de pontos críticos.

A demonstração do seguinte resultado, conhecido como Lema de Morse, pode ser encontrada em [43].

Teorema 1.19. Seja $p$ um ponto crítico não degenerado de uma aplicação $f: D^{2} \rightarrow \mathbb{R}$ de classe $C^{2}$. Então existe um sistema de coordenadas $\left(x_{1}, x_{2}\right)$ em torno de $p$ tal que uma das seguintes alternativas ocorre: 
1) $f\left(x_{1}, x_{2}\right)=f(p)+x_{1}^{2}+x_{2}^{2}$;

2) $f\left(x_{1}, x_{2}\right)=f(p)-x_{1}^{2}-x_{2}^{2}$;

3) $f\left(x_{1}, x_{2}\right)=f(p)+x_{1}^{2}-x_{2}^{2}$.

Deste resultado concluímos que as curvas de nível de uma aplicação de Morse em torno de seus pontos críticos têm o mesmo comportamento que as órbitas de um campo vetorial em torno de um ponto singular tipo centro ou tipo sela hiperbólica.

Seja $M$ uma variedade diferenciável e $\mathcal{F}$ uma folheação de codimensão 1 e classe $C^{2}$ em $M$.

Definição 1.20. Uma aplicação $f: D^{2} \rightarrow M$ de classe $C^{2}$ está em posição geral com respeito a $\mathcal{F}$, se para todo $p \in M$ existe uma carta $(U, \varphi) \in \mathcal{F}$ contendo $p$ tal que se $\pi: U \rightarrow \mathbb{R}$ é a projeção ao longo das placas então $\pi \circ f$ é uma função de Morse. Um ponto crítico de $f$ com respeito à folheação $\mathcal{F}$ é um ponto crítico de $\pi \circ f$ para alguma $\operatorname{carta}(U, \varphi) \in \mathcal{F}$.

A demonstração do próximo resultado pode ser encontrada em [10, 11, 44].

Teorema 1.21. Seja $\mathcal{F}$ uma folheação de codimensão 1 de classe $C^{2}$ em uma variedade diferenciável $M$. Seja $f: D^{2} \rightarrow M$ uma aplicação $C^{2}$. Então existe uma aplicação $\eta: D^{2} \rightarrow M$ de classe $C^{2}$ arbitrariamente $C^{2}$-próxima de $f$ tal que

1) $\eta$ está em posição geral com respeito a $\mathcal{F}$;

2) Se $p, q$ são pontos críticos distintos de $\eta$ com respeito a $\mathcal{F}$ então $\eta(p)$ e $\eta(q)$ estão em diferentes folhas de $\mathcal{F}$.

Seja $\mathcal{G}$ uma folheação de $D^{2}$ com finitas singularidades $\left\{p_{1}, \ldots, p_{k}\right\}$, isto é, $\mathcal{G}$ é uma folheação regular em $D^{2} \backslash\left\{p_{1}, \ldots, p_{k}\right\}$ mas não o é em qualquer subconjunto de $D^{2}$ contendo $p_{i}$, para qualquer $i=1, \ldots, k$. Suponha que $\mathcal{G}$ é transversal ao bordo $\partial D^{2}$. Dizemos que $\mathcal{G}$ é $C^{r}$ localmente orientável se para todo $p \in D^{2}$ existem uma vizinhança $U$ de $p$ e um campo de vetores $X$ de classe $C^{r}$ em $U$ tal que se $q \in U$ é um ponto não singular de $\mathcal{G}$, então $X(q) \neq 0$ e é tangente à folha de $\mathcal{G}$ passando por $q$ e se $q$ é um ponto singular de $\mathcal{G}$ então $X(q)=0$. Dizemos que $\mathcal{G}$ é $C^{r}$ orientável se existir um campo $X$ como acima, definido em todo disco $D^{2}$. O seguinte resultado está provado em [10]:

Proposição 1.22. Se $\mathcal{G}$ é uma folheação com um número finito de singularidades em $D^{2}$ $C^{r}$ localmente orientável, então $\mathcal{G}$ é $C^{r}$ orientável. 


\subsubsection{Folheações do plano}

No caso particular em que a variedade folheada é o plano $\mathbb{R}^{2}$, existe uma classificação completa, a menos de equivalência topológica, das folheações regulares realizada por Kaplan [37] e Markus [39], indicamos também o livro de Wang [60] onde é feita uma compilação destes resultados. A técnica deles para estudar a estrutura destas folheações se baseia na decomposição do plano em componentes ou regiões, nas quais a folheação é homogênea em certo sentido.

Como vamos usar ostensivamente tal classificação no Capítulo2, vamos explicar sucintamente seus principais aspectos. Para isto, introduzimos o conceito de separatrizes e regiões canônicas de folheações regulares no plano. Vale lembrar que vamos usar o disco unitário $D^{2}$ como modelo topológico do plano $\mathbb{R}^{2}$.

Seja $\mathcal{F}$ uma folheação do disco unitário $D^{2}$. Note que $\mathcal{F}$ não contém folhas compactas. De fato, uma folha compacta bordaria um disco e desde que a folheação restrita neste disco compacto é orientável (por ser tal disco simplesmente conexo), pelo Teorema de PoincaréBendixon o fluxo associado a esta folheação possuiria uma singularidade. Portanto, toda folha $L$ de $\mathcal{F}$ é homeomorfa a $\mathbb{R}$ e $\bar{L} \backslash L \subset \partial \bar{D}^{2}$. Pode-se mostrar que toda folha no espaço das folhas $D^{2} / \mathcal{F}$ é fechada com respeito à topologia quociente.

Assim, temos que duas folhas $L_{1}, L_{2} \in \mathcal{F}$ limitam uma única região do disco $D^{2}$. Isto nos permite introduzir uma nova topologia no espaço das folhas $D^{2} / \mathcal{F}$ cujos abertos básicos serão as regiões limitada por duas folhas. Não é difícil encontrar exemplos de abertos da topologia quociente que não são abertos nesta nova topologia, por isto ela será chamada topologia grossa das folhas. É claro que com relação à topologia grossa nem toda folha é necessariamente fechada. Considere pois o conjunto $S_{0}$ das folhas que não são fechadas nesta topologia.

Definição 1.23. Uma folha contida no fecho grosso de $S_{0}$ será chamada uma separatriz de $\mathcal{F}$. Uma folha l contida no fecho de $\mathbb{S}_{0} \backslash\{l\}$ é chamada separatriz limite de $\mathcal{F}$.

Para ilustrar tais definições considere a folheação dada na Figura 1.1, onde há duas separatrizes (em negrito). Note que enquanto na topologia quociente cada folha é fechada, no exemplo da figura o fecho grosso de cada separatriz contém exatamente ela mesma e a outra separatriz.

Por definição, o conjunto de todas as separatrizes é um subconjunto fechado de $D^{2}$ na topologia grossa de $D^{2} / \mathcal{F}$, portanto é também fechado em relação à topologia quociente.

Observação 1.24. Note que a definição de separatrizes para folheações regulares de $\mathbb{R}^{2}$ dada na presente seção é um caso particular das separatrizes para campos vetoriais 


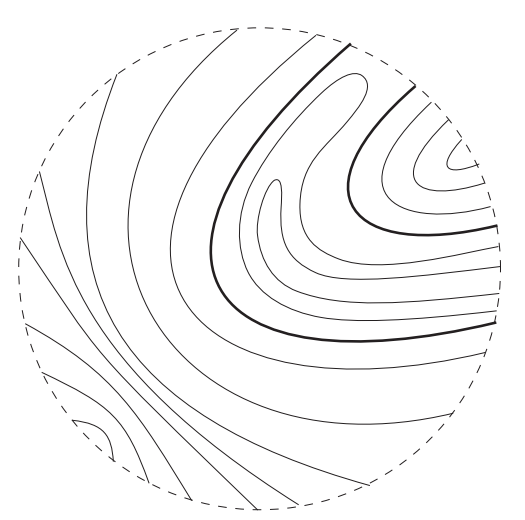

Figura 1.1: Separatrizes

em $\mathfrak{X}^{1}\left(\mathbb{R}^{2}\right)$ introduzidas, na Seção 1.1. De fato, dada uma $C^{2}$-folheação $\mathcal{F}$ do plano, é possível escolher um campo $X \in \mathfrak{X}^{1}\left(\mathbb{R}^{2}\right)$ tal que as órbitas do retrato de fase de $X$ coincidem com as folhas de $\mathcal{F}$. Note que $X$ não possui pontos singulares. Seja $\gamma$ uma separatriz da folheação $\mathcal{F}$, se $\gamma$ não for uma separatriz do campo $X$ então ela possui uma vizinhança paralela $V$ satisfazendo a) e b) da Definição 1.5. Como $X$ não possui singularidades, $\gamma_{-}=\gamma_{+}=\varnothing$ e a região $V$ é necessariamente do tipo faixa. Isto implica que $\gamma$ é fechada na topologia grossa e portanto não é uma separatriz de $\mathcal{F}$. Com efeito, dada uma folha qualquer $L$ de $\mathcal{F}$, podemos tomar uma vizinhança $V_{\gamma}$ de $\gamma$ na topologia grossa tal que $V_{\gamma} \cap L=\varnothing$, bastando para isto tomar uma subfaixa suficientemente pequena de $V$, a qual pela Definição 1.5 é um aberto básico da topologia grossa.

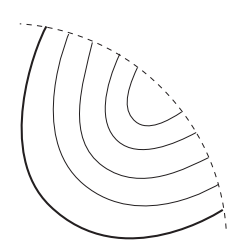

(I)

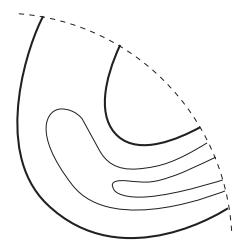

(II)

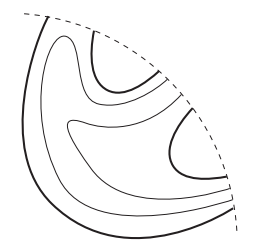

(III)

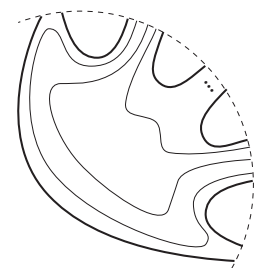

(IV)

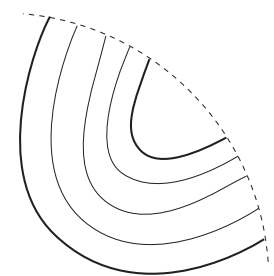

(V)

Figura 1.2: Cinco tipos básicos de regiões canônicas

Definição 1.25. Uma componente conexa do complemento da união das separatrizes em 
$D^{2}$ é chamada de região canônica. Duas regiões canônicas $C_{1}$ e $C_{2}$ são ditas adjacentes se existe uma separatriz $L$ tal que $C_{1} \cup L \cup C_{2}$ é uma região conexa de $D^{2}$.

Pela Observação 1.24 segue que a definição de regiões canônicas para folheações do $\mathbb{R}^{2}$ é um caso particular do conceito homônimo para campos vetoriais do plano. Segundo Kaplan [37] as regiões canônicas que constituem uma folheação do plano podem ser classificadas em cinco tipos, descritos na Figura 1.2, onde as separatrizes estão representadas por linhas ligeiramente mais grossas. Tal figura é uma adaptação a partir dos cinco diagramas de Kaplan básicos apresentados por Wang em [60]. Note que a região do tipo (IV) é a mais geral contendo ao menos quatro e podendo possuir infinitas separatrizes.

\subsection{Sistemas discretos}

Introduziremos aqui alguns conceitos e resultados acerca dos sistemas dinâmicos discretos definidos em $\mathbb{R}^{n}$, os quais podem ser encontrados em qualquer literatura sobre o tema, tais como [42, 54].

Estamos interessados em estudar os difeomorfismos $f: \mathbb{R}^{n} \rightarrow \mathbb{R}^{n}$ de classe $C^{1}$. Denotaremos por $\operatorname{Diff}\left(\mathbb{R}^{n}\right)$ o conjunto de tais difeomorfismos. Dado $f \in \operatorname{Diff}\left(\mathbb{R}^{n}\right)$. Definimos como órbita de um ponto $p \in \mathbb{R}^{n}$ o conjunto $\left\{f^{k}(p) ; k \in \mathbb{Z}\right\}$. Quando tal conjunto é finito, dizemos que p é um ponto periódico e o menor inteiro $k>0$ tal que $f^{k}(p)=p$ é chamado de período de $p$. Se $f(p)=p$, dizemos que $p$ é um ponto fixo. Dizemos que um ponto $q \in \mathbb{R}^{n}$ pertence ao conjunto $\omega$-limite de $p$, denotado por $\omega(p)$, quando existe uma sequência de inteiros $k_{i} \rightarrow \infty$ tal que $f^{k_{i}}(p) \rightarrow q$. Se $y$ é um ponto da órbita de $p$, então $\omega(y)=\omega(p)$. É possível mostrar que $\omega(p)$ é fechado e $f$-invariante, ou seja, $f^{k}(q) \in \omega(p)$ para todo $q \in \omega(p)$ e $k \in \mathbb{Z}$. Se $p$ é um ponto periódico então $\omega(p)$ coincide com a órbita de $p$. Analogamente definimos o conjunto $\alpha$-limite de $p$, denotado por $\alpha(p)$, como sendo o $\omega$-limite de $p$ para o difeomorfismo inverso $f^{-1}$. É claro que as propriedades do conjunto $\omega(p)$ mencionadas acima sao válidas também para $\alpha(p)$. Dizemos que $p \in \mathbb{R}^{n}$ é um ponto não errante se para cada vizinhança $V$ de $p$, existe um inteiro $k>1$ e um ponto $q \in V$ tal que $f^{k}(q) \in V$. Denotamos por $\Omega(f)$ o conjunto de todos os pontos não errantes de $f$.

Dados dois difeomorfismos $f, g \in \operatorname{Diff}\left(\mathbb{R}^{n}\right)$ dizemos que $f$ é topologicamente conjugado a $g$ se existir um homeomorfismo $h: \mathbb{R}^{n} \rightarrow \mathbb{R}^{n}$ tal que $h \circ g=f \circ h$. O homeomorfismo $h$ é chamado conjugação entre $f$ e $g$. Este conceito estabelece uma equivalência entre as estruturas de órbitas de dois difeomorfismos.

Se $p \in \mathbb{R}^{n}$ é um ponto fixo de $f \in \operatorname{Diff}\left(\mathbb{R}^{n}\right)$, dizemos que $p$ é um ponto fixo hiperbólico de $f$ se a derivada $D f_{p}: \mathbb{R}^{n} \rightarrow \mathbb{R}^{n}$ não possuir autovalor de norma 1 . Neste caso, o número 
de autovalores de $D f_{p}$ que têm norma menor que 1 será chamado índice de estabilidade de $f$ em $p$. Um ponto fixo $p$ é do tipo sela hiperbólica se seu índice de estabilidade é maior do que zero e menor que $n$.

O seguinte resultado devido a Hartman e Grobman diz que um difeomorfismo $f \in$ $\operatorname{Diff}\left(\mathbb{R}^{n}\right)$ é localmente conjugado à sua parte linear num ponto fixo hiperbólico.

Teorema 1.26 (Hartman-Grobman). Se $f \in D i f f\left(\mathbb{R}^{n}\right)$ e p é um ponto fixo hiperbólico de $f$, então existem vizinhanças $V$ de $p$ e $U$ da origem $O \in \mathbb{R}^{n}$ e um homeomorfismo $h: U \rightarrow V$ tal que $h \circ D f_{p}=f \circ h$.

Sejam $f \in \operatorname{Diff}\left(\mathbb{R}^{n}\right)$ e $p$ um ponto fixo hiperbólico de $f$. O conjunto $W^{s}(p)$ (resp. $\left.W^{u}(p)\right)$ dos pontos de $\mathbb{R}^{n}$ que têm $p$ como $\omega$-limite (resp. $\alpha$-limite) é chamado variedade estável (resp. instável) de $p$. Não é difícil ver que $W^{s}(p)$ e $W^{u}(p)$ são conjuntos $f$-invariantes.

Terminamos nossas preliminares discreta com o conceito de difeomorfismos que preservam orientação. O espaço vetorial $\mathbb{R}^{n}$ possui uma orientação positiva dada por sua base canônica $\left\{e_{1}, \ldots, e_{n}\right\}$. Uma base qualquer $\left\{u_{1}, \ldots, u_{n}\right\}$ de $\mathbb{R}^{n}$ é dita positiva (resp. negativa) se a matriz mudança desta base para a base canônica tiver determinante positivo (resp. negativo). Dizemos que um isomorfismo linear $T: \mathbb{R}^{n} \rightarrow \mathbb{R}^{n}$ preserva orientação se $\left\{T e_{1}, \ldots, T e_{n}\right\}$ for uma base positiva ou, equivalentemente, se o determinante da matriz $[T]$, da transformação $T$ em relação à base canônica, for positivo. Um difeomorfismo $f: \mathbb{R}^{n} \rightarrow \mathbb{R}^{n}$ preserva orientação se o isomorfismo $D f_{p}: \mathbb{R}^{n} \rightarrow \mathbb{R}^{n}$ preserva orientação, para todo $p \in \mathbb{R}^{n}$. Note que o fato de $f$ ser difeomorfismo implica que $\operatorname{det}\left(D f_{p}\right) \neq 0$, $\forall p \in \mathbb{R}^{n}$ e portanto $\operatorname{det}\left(D f_{p}\right)>0$ ou $<0, \forall p \in \mathbb{R}^{n}$. Isto significa que de fato basta analisar o sinal do determinante $\operatorname{det}\left(D f_{p}\right)$ num ponto $p$ qualquer $\operatorname{de} \mathbb{R}^{n}$. Se $f \in \operatorname{Diff}\left(\mathbb{R}^{n}\right)$ não preserva orientação, é usual dizer que $f$ reverte orientação. 


\section{Sistemas dinâmicos bidimensionais com um único ponto de equilíbrio}

Na primeira seção deste capítulo provaremos os Teoremas A e B, e na seção seguinte estabeleceremos os Teoremas C e D enunciados na Introdução.

\subsection{Caso contínuo}

Como anteriormente, denotaremos por $\mathfrak{X}_{s}^{1}\left(\mathbb{R}^{2}\right)$, o conjunto dos campos vetoriais $X$ : $\mathbb{R}^{2} \rightarrow \mathbb{R}^{2}$, de classe $C^{1}$, que possuem um único ponto singular na origem $O$ que é do tipo sela hiperbólica. Dado $X \in \mathfrak{X}_{s}^{1}\left(\mathbb{R}^{2}\right)$, desde que $X$ não possui outros pontos singulares além da sela hiperbólica $O$, pelo teorema de Poincaré-Bendixon [42], não há órbitas fechadas associadas a este campo. Além disto, $\omega(\gamma)=\alpha(\gamma)=\varnothing$ para cada trajetória $\gamma$ de $X$, exceto para o ponto singular e suas separatrizes. Desta forma, podemos ordenar as quatro separatrizes $s_{0}, s_{1}, s_{2}$ e $s_{3}$ da sela, considerando o sentido positivo de rotação como na Figura 2.1.

Denotaremos por $W_{i}$ a região aberta de $\mathbb{R}^{2}$ cuja fronteira é $s_{i} \cup s_{j} \cup O$, onde $j=(i+1)$ mod 4 , e que não contém outra separatriz de $O$. Note que tais regiões são homeomorfas a $\mathbb{R}^{2}$. A folheação regular de $W_{i}$ associada ao campo $X$ será denotada por $\mathcal{F}\left(W_{i}\right)$.

\subsubsection{Sela global}

Uma vez que as regiões $W_{i}$, para $i=0,1,2,3$, são homeomorfas ao plano e as folheações $\mathcal{F}\left(W_{i}\right)$ são sem singularidades, pode-se aplicar a classificação completa, a menos de equivalência topológica, das folheações regulares de $\mathbb{R}^{2}$ realizada por Kaplan [37] e Markus [39] e descrita no capítulo anterior. O próximo exemplo ilustra que dado $X \in \mathfrak{X}_{s}^{1}\left(\mathbb{R}^{2}\right)$, em 


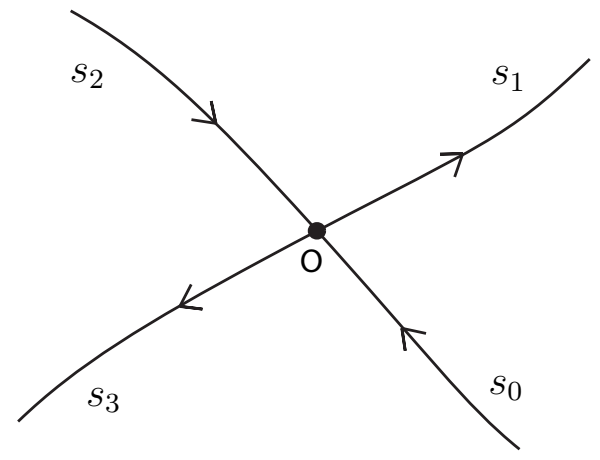

Figura 2.1: Separatrizes positivamente ordenadas de uma sela hiperbólica

geral, não é verdade que as folheações $\mathcal{F}\left(W_{i}\right)$ são triviais, nem mesmo no caso polinomial.

Exemplo 2.1. Considere os campos vetoriais $X_{1}(x, y)=\left(x-x^{2} y(x-y),-y-x y^{2}(x-y)\right)$ e $X_{2}(x, y)=\left(x-x^{2} y(x-y)(2 y-x),-y-1.1 x y^{2}(x-y)(2 y-x)\right)$ os quais pertencem a $\mathfrak{X}_{s}^{1}\left(\mathbb{R}^{2}\right)$. Usando o programa $\mathrm{P} 4$ podemos concluir que nem sempre as folheações nas regiões $W_{i}$ serão triviais como pode ser visto na Figura 2.2. Para descrição e obtenção do software P4 veja [4].
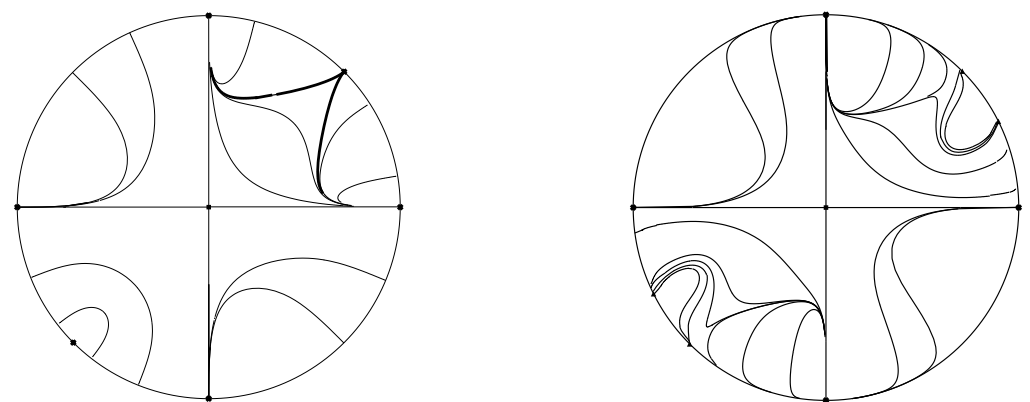

Figura 2.2: $\quad$ Retrato de fase de $X_{1}$ e $X_{2}$ do Exemplo 2.1

Na presente subseção e na seguinte, discutiremos condições sobre as quais um campo vetorial em $\mathfrak{X}_{s}^{1}\left(\mathbb{R}^{2}\right)$ tenha as folheações em $W_{i}$ triviais, para todo $i \in\{0,1,2,3\}$.

No que segue serão introduzidas definições para o caso particular de campos em $\mathfrak{X}_{s}^{1}\left(\mathbb{R}^{2}\right)$, as quais se mostrarão úteis no desenvolvimento deste trabalho.

Definição 2.2. Dado $X$ um campo vetorial em $\mathfrak{X}_{s}^{1}\left(\mathbb{R}^{2}\right)$, a região canônica de $W_{i}$ cujo fecho contém a singularidade $O$ será chamada região canônica inicial de $W_{i}$. Para tal região canônica, definiremos $s_{i} \cup s_{j} \cup O$, com $j=(i+1) \bmod 4$, como sua separatriz inicial. 
É claro que, $s_{i} \cup s_{j} \cup O \not \subset W_{i}$ e portanto tal união não é uma separatriz no sentido que definimos na Seção 1.1, porém para simplificar a seguinte definição por recorrência, chamamos-la separatriz inicial.

Definição 2.3. Dado $X \in \mathfrak{X}_{s}^{1}\left(\mathbb{R}^{2}\right)$, a região canônica inicial de $W_{i}$ é dita região canônica de nível 1. Uma região canônica será de nível 2 se possuir uma separatriz em comum com à região canônica inicial. Indutivamente, uma região canônica será de nível $n$ se tiver uma separatriz em comum com uma região canônica de nível $n-1$.

Definição 2.4. Dados $X \in \mathfrak{X}_{s}^{1}\left(\mathbb{R}^{2}\right)$ e uma região canônica $C$ em $W_{i}$ de nível $n$, a separatriz de $C$ que é comum à região canônica de nível $n-1$ adjacente a $C$ será chamada separatriz inicial de $C$.

Na seguinte figura mostra-se duas folheações em $W_{i}$ associadas a possíveis campos vetoriais em $\mathfrak{X}_{s}^{1}\left(\mathbb{R}^{2}\right)$, onde aparecem regiões canônicas de níveis 1 a 4 . À esquerda, a região canônica inicial é do tipo (II), existem duas regiões canônicas de nível 2: uma do tipo (III) e a outra do tipo (IV). Temos seis regiões de nível 3, das quais cinco são do tipo (I) e uma de tipo (III). Finalmente, existem duas de nível 4, ambas do tipo (I).
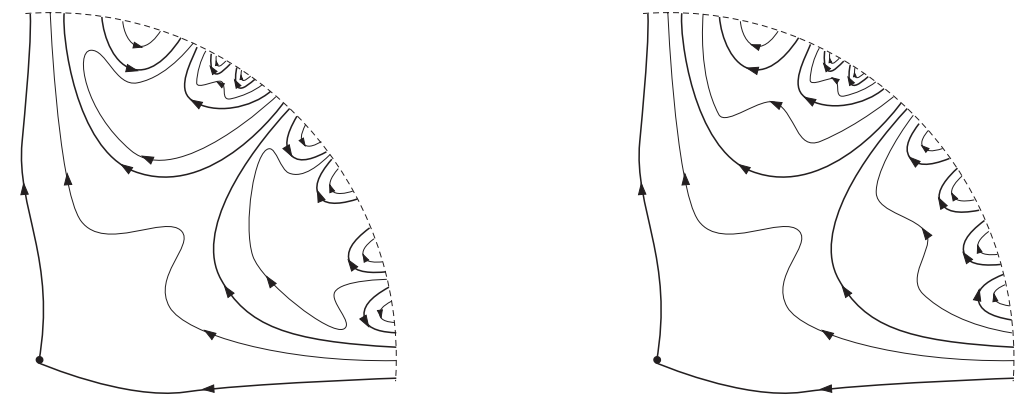

Figura 2.3: Exemplos de $\mathcal{F}\left(W_{i}\right)$

Introduzimos agora o conceito de setor pseudo-hiperbólico no infinito para campos planos e enunciamos um resultado dando condições para a não existência de tais setores. Tais noções foram extraídas de [1].

Dado um campo vetorial $X=(f, g)$, seja $X^{*}=(-g, f)$ o campo vetorial ortogonal a $X$. Será usado a mesma notação de intervalos de $\mathbb{R}$ para arcos orientados de trajetórias $[p, q],[p, q), \ldots \quad\left(\operatorname{resp} . \quad[p, q]^{*},[p, q)^{*}, \ldots\right)$ de $X$ (resp. $\left.X^{*}\right)$, ligando os pontos $p$ e $q . \quad \mathrm{A}$ orientação destes arcos é a induzida pelos campos $X$ (resp. $X^{*}$ ).

Definição 2.5. [Setor pseudo-hiperbólico no infinito] Seja $X \in \mathfrak{X}^{1}\left(\mathbb{R}^{2} \backslash \bar{D}_{\sigma}\right)$ e $S=$ $S\left(p_{1}, p_{2} ; q_{1}, q_{2},\left\{\sigma_{i}\right\}\right) \subset \mathbb{R}^{2} \backslash \bar{D}_{\sigma}$ a região não limitada cuja fronteira $\partial S$ é constituída de 
duas semi-trajetórias não limitadas $\left[q_{1}, \infty\right)$ e $\left(\infty, q_{2}\right]$ de $X$, um arco compacto de trajetória $\left[p_{1}, p_{2}\right]$ de $X$, dois arcos de trajetória $\left[p_{1}, q_{1}\right]^{*},\left[p_{2}, q_{2}\right]^{*}$ de $X^{*}$ e um conjunto no máximo enumerável (podendo ser vazio) de trajetórias $\sigma_{1}, \sigma_{2}, \cdots, \sigma_{n}, \cdots$ que começam e terminam no $\infty$ (veja Figura 2.4). Tal região é um setor pseudo-hiperbólico de $X$ no infinito se as seguintes condições forem satisfeitas:

1) Para cada $z \in\left[p_{1}, q_{1}\right)^{*}$, existe um arco de trajetória $[z, \pi(z)] \subset S$ de $X$ começando em $z \in\left[p_{1}, q_{1}\right]^{*}$ e terminando em $\pi(z) \in\left[p_{2}, q_{2}\right]^{*}$;

2) $\overline{\bigcup_{z \in\left[p_{1}, q_{1}\right)}[z, \pi(z)]}=S$.

A seguinte notação será usada para a parte não limitada de $\partial S$ :

$$
\partial_{+} S=\left[q_{1}, \infty\right) \cup\left(\infty, q_{2}\right] \cup \bigcup_{i=1}^{\infty} \sigma_{i} \subset \partial S .
$$

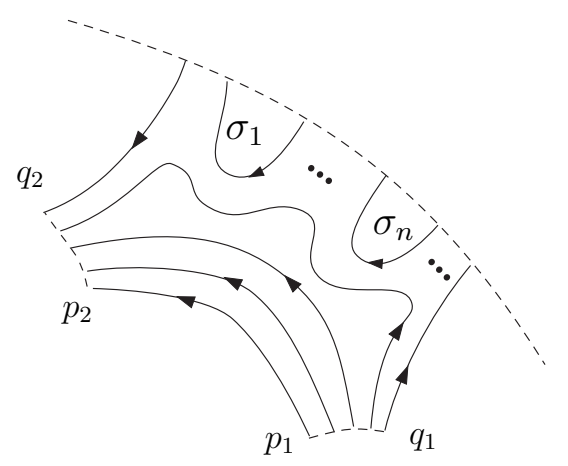

Figura 2.4: Setor pseudo-hiperbólico no infinito

Observação 2.6. O leitor pode observar a Figura 1.2 para concluir que se uma folheação no plano induzida por um campo vetorial $X \in \mathfrak{X}^{1}\left(\mathbb{R}^{2}\right)$, sem pontos singulares, tem uma região canônica do tipo (II), (III) ou (IV), então X possui um setor pseudo-hiperbólico no infinito.

Definição 2.7. Sejam A um subconjunto Lebesgue mensurável de $\mathbb{R}^{2}$ e $f: A \rightarrow \mathbb{R}$ uma função mensurável. Considere a seguinte notação:

$$
f^{+}(x)=\max \{f(x), 0\} \quad \text { e } f^{-}(x)=\max \{-f(x), 0\} .
$$


Uma função $f: A \rightarrow \mathbb{R}$ será dita quase-integrável se

$$
\min \left\{\int_{A} f^{+} d \lambda, \int_{A} f^{-} d \lambda\right\}<\infty
$$

neste caso define-se

$$
\int_{A} f d \lambda=\int_{A} f^{+} d \lambda-\int_{A} f^{-} d \lambda
$$

o qual é um valor bem definido na reta real estendida $[-\infty, \infty]$.

Se $X \in \mathfrak{X}^{1}(U)$, escrevemos por $\operatorname{Div}(X): U \rightarrow \mathbb{R}$ a função (contínua) que a cada $p \in U$ associa o valor real $\operatorname{Traço}\left(D_{p} X\right)$. Neste contexto, consideramos a seguinte notação

$$
\mathfrak{D}^{1}(U)=\left\{X \in \mathfrak{X}^{1}(U) ; \operatorname{Div}(X) \text { é quase-integrável em } U\right\} \text {. }
$$

A demonstração da seguinte proposição pode ser encontrada em [1] num contexto um pouco mais geral. De fato, os autores provam tal resultado para campos definidos em uma vizinhança do infinito. Desde que, à primeira vista, a hipótese (2.1) causa certo desconforto por falta de intuitividade, inserimos aqui a prova deste resultado, no intuito de explicitar a relação de tal hipótese com a não existência de setores pseudo-hiperbólicos no $\infty$, .

Proposição 2.8. Seja $X \in \mathfrak{D}^{1}\left(\mathbb{R}^{2}\right)$, tal que para algum $r>0$

$$
\int_{r}^{\infty} \inf \{\|X(p)\| ;\|p\|=s\} d s=\infty
$$

Então X não possui setores pseudo-hiperbólicos no infinito.

Dem.: A idéia desta demonstração é utilizar o teorema de Green para provar que se $X$ possui um setor pseudo-hiperbólico no infinito, então existe um caminho do plano com uma das extremidades em $\infty$ tal que a integral da norma do campo neste caminho é finita, implicando uma contradição com a igualdade (2.1).

Suponha que $X=(f, g)$ tem um setor pseudo-hiperbólico no infinito. Afirmamos que tal setor contém uma região $R$ cuja fronteira é a união de uma semi-trajetória $\left[q_{1}, \infty\right)$ de $X$, um arco compacto de trajetória $\left[p_{1}, p_{\tau_{0}}\right]$ de $X$, um arco compacto de trajetória $\left[p_{1}, q_{1}\right]^{*}$ de $X^{*}$ e uma semi-trajetória $\left[p_{\tau_{0}}, \infty\right)^{*}$ de $X^{*}$. Tal situação pode ser visualizada na Figura 2.5. Com efeito, tome uma parametrização $\tau \rightarrow p_{\tau}$, onde $1 \leq \tau \leq 2$. Seja $\tau_{0} \in(1,2]$ o parâmetro máximo com respeito à seguinte propriedade: para todo $\tau \in\left[1, \tau_{0}\right)$, existe um arco de trajetória $\left[p_{\tau}, q_{\tau}\right]^{*} \subset S$ de $X^{*}$ tal que $\left[p_{\tau}, q_{\tau}\right]^{*} \cap \partial S=\left\{p_{\tau}, q_{\tau}\right\}$, onde $q_{\tau} \in\left[q_{1}, \infty\right)$. 
A família de arcos de trajetória $\left\{\left[p_{\tau}, q_{\tau}\right]^{*} ; 1 \leq \tau \leq \tau_{0}\right\}$ certamente depende continuamente do parâmetro $\tau$. Desde que $X$ e $X^{*}$ são transversais, temos que se

$$
R_{1}=\bigcup_{1 \leq \tau<\tau_{0}}\left[p_{\tau}, q_{\tau}\right]^{*}
$$

então $R_{1} \cap \partial S=\left[p_{1}, q_{1}\right]^{*} \cup\left[q_{1}, \infty\right) \cup\left[p_{1}, p_{\tau_{0}}\right)$, onde $\left[p_{1}, p_{\tau_{0}}\right)$ é o sub-intervalo semi-aberto de $\left[p_{1}, p_{2}\right]$ com pontos finais $p_{1}$ e $p_{\tau_{0}}$. Portanto, $\bar{R}_{1}=R_{1} \cup\left[p_{\tau_{0}}, \infty\right)^{*} \subset S$, onde $\left[p_{\tau_{0}}, \infty\right)^{*}$ denota a semi-trajetória de $X^{*}$ começando em $p_{\tau_{0}}$ e contido em $S$. Isto implica que $R=\bar{R}_{1}=R_{1} \cup\left[p_{\tau_{0}}, \infty\right)^{*}$ é a região requerida. Sendo $\operatorname{Div}(X)$ quase-integrável, considere

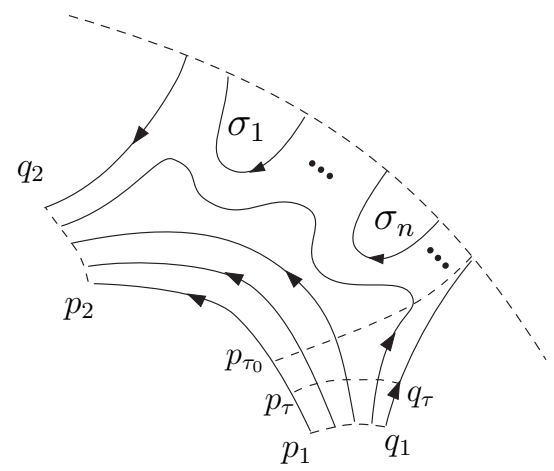

Figura 2.5: Setor pseudo-hiperbólico no infinito

o caso em que $\int_{\mathbb{R}^{2}} \operatorname{Div}(X) \in(-\infty, \infty]$. Por um lado temos que $\int_{R} \operatorname{Div}(X) \in(-\infty, \infty]$ e aplicando o teorema de Green:

$$
\int_{R} \operatorname{Div}(X)=\int_{R}(\partial f / \partial x-\partial(-g) / \partial y)=\int_{\partial R}-g d x+f d y
$$

Note que tal integral por caminhos pode ser naturalmente separada em quatro integrais nos caminhos $\left[p_{1}, p_{\tau_{0}}\right],\left[q_{1}, \infty\right),\left[p_{1}, q_{1}\right]^{*} \mathrm{e}\left[p_{\tau_{0}}, \infty\right)^{*}$. Além disto, as duas primeiras integrais são nulas. De fato, como o caminho $\gamma=\left(\gamma_{1}, \gamma_{2}\right)$ nestas integrais coincidem com o fluxo associado a $X=(f, g)$, temos que $d x=\gamma_{1}^{\prime}(t) d t=f(\gamma(t)) d t$ e $d y=\gamma_{2}^{\prime}(t) d t=g(\gamma(t)) d t$. Por outro lado os dois últimos caminhos coincidem com fluxo ortogonal associado ao campo $X^{*}=(-g, f)$. Logo, nestas integrais temos $d x=-g\left(\phi^{*}(t,).\right) d t$ e $d y=f\left(\phi^{*}(t,).\right) d t$. Portanto podemos reescrever a equação $(2.2)$ como

$$
\int_{R} \operatorname{Div}(X)=\int_{\left[p_{1}, q_{1}\right]^{*}}\left\|X\left(\phi^{*}\left(t, p_{1}\right)\right)\right\|^{2} d t-\int_{\left[p_{\tau_{0}}, \infty\right)^{*}}\left\|X\left(\phi^{*}\left(t, p_{\tau_{0}}\right)\right)\right\|^{2} d t
$$

$\mathrm{Ou}$

$$
\int_{R} \operatorname{Div}(X)=\int_{0}^{s_{1}}\left\|X\left(\phi^{*}\left(t(s), p_{1}\right)\right)\right\| d s-\int_{0}^{\infty}\left\|X\left(\phi^{*}\left(t(s), p_{\tau_{0}}\right)\right)\right\| d s,
$$


onde $d s=\left\|X\left(\phi^{*}(t, p)\right)\right\| d t=\left\|X^{*}\left(\phi^{*}(t, p)\right)\right\| d t=\left\|\partial \phi^{*} / \partial t\left(\phi^{*}(t, p)\right)\right\| d t$ é o elemento de linha e $s_{1}$ é o comprimento do segmento $\left[p_{1}, q_{1}\right]^{*}$.

Pela igualdade 2.1 temos que $\int_{0}^{\infty}\left\|X\left(\phi^{*}\left(t(s), p_{\tau_{0}}\right)\right)\right\| d s=\infty$. Portanto,

$$
\int_{R} \operatorname{Div}(X)=\int_{0}^{s_{1}}\left\|X\left(\phi^{*}\left(t(s), p_{1}\right)\right)\right\| d s-\int_{0}^{\infty}\left\|X\left(\phi^{*}\left(t(s), p_{\tau_{0}}\right)\right)\right\| d s=-\infty,
$$

O que é absurdo pois assumimos $\int_{R} \operatorname{Div}(X) \in(-\infty, \infty]$.

Observe que se considerarmos o caso $\int_{\mathbb{R}^{2}} \operatorname{Div}(X) \in[-\infty, \infty)$, é possível obter uma região $R$ de tal forma que aplicando o teorema de Green como acima obtemos:

$$
\int_{R} \operatorname{Div}(X)=\int_{0}^{\infty}\left\|X\left(\phi^{*}\left(t(s), p_{\tau_{0}}\right)\right)\right\| d s-\int_{0}^{s_{2}}\left\|X\left(\phi^{*}\left(t(s), p_{2}\right)\right)\right\| d s,
$$

onde $s_{2}$ é o comprimento do segmento $\left[p_{2}, q_{2}\right]^{*}$.

Na Subseção 2.1.3 apresentamos alguns exemplos de campos vetoriais $X \in \mathfrak{X}^{1}\left(\mathbb{R}^{2}\right)$ satisfazendo as hipóteses 2.1) e $\operatorname{Div}(X)$ quase-integrável.

Temos portanto as ferramentas necessárias para provar:

Teorema A. Todo campo vetorial em $\mathfrak{X}_{s}^{1}\left(\mathbb{R}^{2}\right) \cap \mathfrak{D}^{1}\left(\mathbb{R}^{2}\right)$ satisfazendo 2.1) é uma sela global, isto é, topologicamente equivalente à sela linear $L(x, y)=(-x, y)$.

Dem.: Pelo Teorema 1.8, é suficiente provar a trivialidade das folheações $\mathcal{F}\left(W_{i}\right)$, para todo $i \in\{0, \ldots, 3\}$. Da Proposição 2.8, não existem setores pseudo-hiperbólicos no infinito para $X$. Este fato, aliado à observação 2.6, implicam que as regiões canônicas na folheação $\mathcal{F}\left(W_{i}\right)$ de $W_{i}$ não podem ser dos tipos (II), (III) nem (IV), ou seja, $\mathcal{F}\left(W_{i}\right)$ é uma combinação de regiões do tipo (I) e (V). Porém, se existisse alguma região canônica do tipo (V), uma das regiões canônicas adjacentes seria necessariamente do tipo (II), (III) ou (IV), o que não pode ocorrer como já foi visto. Portanto $\mathcal{F}\left(W_{i}\right)$ é trivial para todo $i \in\{0, \ldots, 3\}$.

\subsubsection{Sela global e injetividade}

Nesta subseção estamos interessados em obter um resultado análogo ao de MarkusYamabe bidimensional no âmbito das selas globais. Como no caso daquele problema, estabeleceremos uma relação entre injetividade e sela global. Mais especificamente, no 
Corolário 2.13 concluímos que as hipóteses de unicidade de pontos singulares e a condição (2.1) no Teorema A, podem ser substituídas por alguma hipótese que garanta a injetividade do campo vetorial $X$, sempre que $X$ for um difeomorfismo local.

Dado um campo vetorial $X: \mathbb{R}^{2} \rightarrow \mathbb{R}^{2}$ diferenciável, denotamos por $\operatorname{Spec}(X)$ o conjunto dos autovalores de $D X_{p}$ quando $p$ varia em todo $\mathbb{R}^{2}$. Na Introdução, discutimos sobre a seguinte:

Conjetura de Markus-Yamabe: Seja $X: \mathbb{R}^{2} \rightarrow \mathbb{R}^{2}$ um campo vetorial de classe $C^{1}$ tal que $X(O)=O$ e

$$
\operatorname{Spec}(X) \subset\{z \in \mathbb{C} ; \operatorname{Re}(z)<0\} .
$$

Então, $O$ é um atrator global do sistema associado $x^{\prime}=X(x)$.

Dizemos que um campo vetorial $X \in \mathfrak{D}^{1}\left(\mathbb{R}^{2}\right)$ pertence ao conjunto $\mathcal{S} \mathcal{S}$ se possuir um ponto singular hiperbólico do tipo sela e existir $\epsilon>0$ tal que:

$$
\operatorname{Spec}(X) \cap[0, \epsilon)=\varnothing \quad \text { ou } \operatorname{Spec}(X) \cap(-\epsilon, 0]=\varnothing .
$$

Obtemos o seguinte resultado para selas globais, análogo ao problema de MarkusYamabe:

Teorema B. Todo campo vetorial em $\mathcal{S S}$ é sela global.

Observe que enquanto no problema de Markus-Yamabe a condição do $\operatorname{Div}(X)$ ser quase-integrável era consequência da hipótese espectral, aqui tivemos que impó-la. Por outro lado, em Markus-Yamabe pede-se que o espectro esteja do lado direito do plano complexo, em nosso caso uma condição análoga seria que $\operatorname{Spec}\left(D X_{q}\right)=\left\{\lambda_{1}(q)<0, \lambda_{2}(q)>0\right\}$, para todo $q \in \mathbb{R}^{2}$. Porém, isto é uma consequência do ponto singular ser uma sela hiperbólica aliado ao fato de $X$ ser um difeomorfismo local (veja Lema 2.10). Finalmente, ressaltamos que uma vez que a condição $\operatorname{Spec}\left(D X_{q}\right)=\left\{\lambda_{1}(q)<0, \lambda_{2}(q)>0\right\}$, para todo $q \in \mathbb{R}^{2}$, não garante a injetividade de $X$, tivemos que adicionar a hipótese espectral (2.5) (veja Exemplo 2.17).

No Lema 2.11, veremos que a afirmação: se $X \in \mathcal{S S}$ então $X$ é uma sela global, é equivalente à afirmação: se $X \in \mathcal{S S}$ então $X$ é uma aplicação injetiva. Portanto, o Teorema B seguirá do seguinte resultado de injetividade devido a Fernandes, Gutierrez e Rabanal [21]:

Teorema 2.9. Uma aplicação diferenciável $X: \mathbb{R}^{2} \rightarrow \mathbb{R}^{2}$ é injetiva se satisfaz a condição (2.5) para algum $\epsilon>0$. 
No seguinte resultado caracterizamos o $\operatorname{Spec}(X)$ dos difeomorfismos locais do plano em si mesmo que possuem um ponto singular do tipo sela hiperbólica.

Lema 2.10. Seja $X: \mathbb{R}^{2} \rightarrow \mathbb{R}^{2}$ é um difeomorfismo local de classe $C^{1}$. Se $\operatorname{Spec}\left(D X_{p}\right)=$ $\left\{\lambda_{1}(p)<0, \lambda_{2}(p)>0\right\}$ para algum ponto $p$, então $\operatorname{Spec}\left(D X_{q}\right)=\left\{\lambda_{1}(q)<0, \lambda_{2}(q)>0\right\}$, para todo $q \in \mathbb{R}^{2}$.

Dem.: Em $p$ temos que $\operatorname{Spec}\left(D X_{p}\right)=\left\{\lambda_{1}(p)<0, \lambda_{2}(p)>0\right\}$, em particular $\operatorname{det}\left(D X_{p}\right)<$ 0 . Assim, da continuidade da função $\operatorname{det}\left(D X_{q}\right): \mathbb{R}^{2} \rightarrow \mathbb{R}$ em relação a $q$ e do fato de $X$ ser um difeomorfismo local concluímos que $\operatorname{det}\left(D X_{q}\right)<0$ para todo $q \in \mathbb{R}^{2}$. Logo, o polinômio característico de $D X_{q}$, que é dado por $\lambda^{2}-\operatorname{tr}\left(D X_{q}\right) \lambda+\operatorname{det}\left(D X_{q}\right)$, para todo $q \in \mathbb{R}^{2}$, é mônico e tem termo independente sempre negativo. Portanto, suas raízes são sempre reais sendo uma negativa e a outra positiva.

Analogamente ao que foi feito por Olech [46], no seguinte lema estabelecemos uma equivalência entre injetividade e sela global para campos em $\mathcal{S} \mathcal{S}$ :

Lema 2.11. As seguintes afirmações são equivalentes:

1) $X \in \mathcal{S S}$ implica que $X$ é uma sela global;

2) $X \in \mathcal{S S}$ implica que a aplicação $X: \mathbb{R}^{2} \rightarrow \mathbb{R}^{2}$ é injetiva.

Dem.: 1) $\Rightarrow 2$ ) Suponha por contradição que $X$ é não injetiva. Isto é, existem $q_{1}, q_{2}, a \in$ $\mathbb{R}^{2}$ tais que $q_{1} \neq q_{2}$ e $X\left(q_{1}\right)=X\left(q_{2}\right)=a$. Defina o novo campo vetorial $Y=X-a$ : $\mathbb{R}^{2} \rightarrow \mathbb{R}^{2}$. Note que $Y$ é de classe $C^{1}, \operatorname{Div}(Y)=\operatorname{Div}(X)$ é quase-integrável, $\operatorname{Spec}(Y)=$ $\operatorname{Spec}(X-a)=\operatorname{Spec}(X)$ e, pelo Lema 2.10, o ponto singular $q_{1}$ do campo $Y$ é uma sela hiperbólica e portanto $Y \in \mathcal{S S}$. Como estamos assumindo que o item 1) é verdadeiro, temos que $Y$ é uma sela global, o qual é uma contradição com a existência de outro ponto singular $Y\left(q_{2}\right)=O$.

$2) \Rightarrow 1$ ) Desde que $X$ é injetivo, e sendo $q$ uma sela hiperbólica deste campo, temos que $X(q)=O$ é o único ponto singular de $X$ e portanto $Y(p)=X(p-q) \in \mathfrak{X}_{s}^{1}\left(\mathbb{R}^{2}\right) \cap \mathfrak{D}^{1}\left(\mathbb{R}^{2}\right)$. Pelo Teorema A basta mostrar que, para algum $r>0, X$ satisfaz a condição (2.1) da Proposição 2.8. Para isto mostraremos primeiro que

(a) Existem $r>0$ e $\delta>0$ tais que $\|X(p)\|>\delta$ sempre que $\|p\|>r$.

Como $X$ é um difeomorfismo local injetivo, temos que $X$ é um difeomorfismo global sobre sua imagem. Portanto, a imagem $X\left(\mathbb{R}^{2}\right)$ é um aberto de $\mathbb{R}^{2}$ que contém a origem $O$, pois 
$X(q)=O$. Usando o fato de que um difeomorfismo leva a fronteira de seu domínio na fronteira de sua imagem e que $O$ está no interior da imagem $X\left(\mathbb{R}^{2}\right)$, pode-se concluir que, para algum $r>0$ suficientemente grande, as imagens dos pontos $p \in \mathbb{R}^{2}$, tais que $\|p\|>r$, estarão a uma distância positiva de $O$, o que equivale a dizer que existe um $\delta>0$ tal que se $\|p\|>r$ então $\|X(p)\|>\delta$.

Da afirmação (a) temos que

$$
\int_{r}^{\infty} \inf \{\|X(p)\| ;\|p\|=s\} d s \geq \delta \int_{r}^{\infty} d s=\infty
$$

ou seja, a condição (2.1) da Proposição 2.8 é satisfeita.

Obtemos assim a prova do Teorema B:

Dem.: Desde que um campo vetorial $X \in \mathcal{S S}$, em particular, satisfaz (2.5), pelo Teorema 2.9 segue que $X$ é injetivo e portanto uma sela global pelo Lema 2.11 .

Note que na segunda parte da demonstração do Lema 2.11, o fato essencial para provar que $X$ satisfaz a condição (2.1) é a injetividade de $X$. Portanto, no caso de difeomorfismos locais a injetividade é suficiente para garantir que $X \in \mathfrak{X}_{s}^{1}\left(\mathbb{R}^{2}\right) \cap \mathfrak{D}^{1}\left(\mathbb{R}^{2}\right)$ é sela global. Este critério é um tanto interessante dada a vasta gama de resultados garantindo injetividade de difeomorfismos locais no plano. A seguir listamos alguns destes:

Teorema 2.12. Todo difeomorfismo local $X: \mathbb{R}^{2} \rightarrow \mathbb{R}^{2}$ satisfazendo alguma das seguintes propriedades é injetivo:

1) Fernandes, Gutierrez e Rabanal [21]: $X$ diferenciável e $\operatorname{Spec}(X) \cap[0, \epsilon)=\varnothing$ ou $\operatorname{Spec}(X) \cap(-\epsilon, 0]=\varnothing$ para algum $\epsilon>0$.

2) Braun e dos Santos Filho [9]: $X=(f, g)$ polinomial com o grau de $f$ ou $g$ menor ou igual a 3 (em particular os campos quadráticos);

3) Parthasarathy [47]: $X=(f, g)$ de classe $C^{1}$ tal que uma das seguintes regiões seja limitada em $\mathbb{R}^{2}$ :

i) $\left\{(x, y) ; \frac{\partial f}{\partial x} \frac{\partial f}{\partial y}<0\right\}$;

ii) $\left\{(x, y) ; \frac{\partial f}{\partial y} \frac{\partial g}{\partial x}<0\right\}$;

iii) $\left\{(x, y) ; \frac{\partial f}{\partial x} \frac{\partial f}{\partial y}>0\right\}$. 
(em particular se $\frac{\partial f}{\partial x} \frac{\partial f}{\partial y} \geq$ ou $\left.\leq 0\right)$;

4) Coomes [16]: $X$ de classe $C^{1}$ e a derivada de $X$ é triangular superior ou inferior.

Como consequência do Lema 2.11 e do Teorema 2.12 temos:

Corolário 2.13. Seja $X \in \mathfrak{D}^{1}\left(\mathbb{R}^{2}\right)$ um difeomorfismo local com um ponto singular do tipo sela hiperbólica. Então X é uma sela global sempre que satisfizer alguma das propriedades relacionadas no Teorema 2.12.

\subsubsection{Exemplos}

Exemplo 2.14. O campo vetorial $X(x, y)=\left(-(x+1)^{3}+1,(x+1)^{2}(y+1)-1\right)$ adaptado de [50] satisfaz todas hipóteses do Teorema A e portanto é uma sela global. Porém não satisfaz as condições do Teorema B, uma vez que não é um difeomorfismo local.

Exemplo 2.15. Os exemplos: $X_{1}(x, y)=\left(x-x^{2} y(x-y),-y-x y^{2}(x-y)\right)$ e $X_{2}(x, y)=$ $\left(x-x^{2} y(x-y)(2 y-x),-y-1.1 x y^{2}(x-y)(2 y-x)\right)$ já enunciados em 2.1, ilustram que para um campo $X: \mathbb{R}^{2} \rightarrow \mathbb{R}^{2}$ ser uma sela global não é suficiente que $X \in \mathfrak{X}_{s}^{1}\left(\mathbb{R}^{2}\right)$, isto é, que seja de classe $C^{1}$ e tenha um único ponto singular que é do tipo sela hiperbólica.

Exemplo 2.16. O seguinte exemplo nos dá uma família de campos satisfazendo as hipóteses do Corolário 2.13. Seja $X(x, y)=(a x, b y+g(x))$, onde $a>0, b<0$ e $g: \mathbb{R} \rightarrow \mathbb{R}$ é uma função de classe $C^{1}$ tal que $g(0)=0$. Não é difícil ver que $X \in \mathfrak{X}_{s}^{1}\left(\mathbb{R}^{2}\right)$ e como $\operatorname{Div}(X)=a+b$ temos que tal função é quase-integrável. Além disto, $\operatorname{Spec}(X)=\{a, b\}$ e portanto $X$ satisfaz os itens 1), 3iii) e 4) do Teorema 2.12. Logo, $X$ é sela global.

Exemplo 2.17. O campo vetorial $X(x, y)=\left(-2 e^{x}+3 y^{2}-1, y e^{x}-y^{3}\right)$, adaptado de [47], mostra que mesmo que $X$ seja difeomorfismo local de classe $C^{\infty}$, tenha $\operatorname{Div}(X)$ quaseintegrável e satisfaça a condição (2.1), não é verdade que $X$ seja sela global. Na Figura 2.6 esboçamos seu retrato de fase.

De fato, neste caso, $X(0,1)=X(0,-1)=O$. Isto mostra que não é possível relaxar a hipótese 2.5 no Teorema B para $\operatorname{Spec}\left(D X_{p}\right)=\left\{\lambda_{1}(p)<0, \lambda_{2}(p)>0\right\}$, para todo $p \in \mathbb{R}^{2}$, o que seria uma hipótese análoga ao do problema de Markus-Yamabe.

Este exemplo também mostra a necessidade da unicidade de pontos singulares no Teorema A. Tanto no Teorema B quanto no Corolário 2.13 tal unicidade é garantida pela injetividade. 


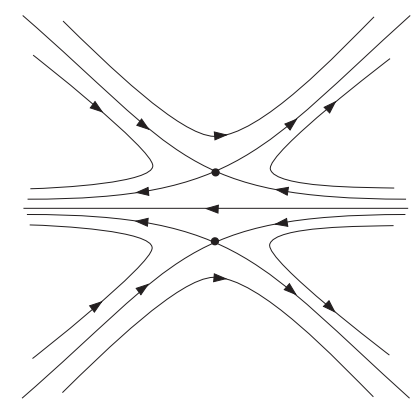

Figura 2.6: Retrato de fase do Exemplo 2.17

Exemplo 2.18. O campo vetorial $X(x, y)=\left(-\left(x^{3} / 3+x\left(y^{2}-1 / 2\right)-y\right), x+y\right)$ é um difeomorfismo local polinomial com $\operatorname{Div}(X)$ quase-integrável e claramente satisfaz o item 2) do Teorema 2.12. Portanto, $X$ é sela global pelo Corolário 2.13.

Exemplo 2.19. Das condições do Teorema A, a única que o campo vetorial dado por $X(x, y)=\left(x-1 / 2 x^{2},-y\right)$ não satisfaz é que $\operatorname{Div}(X)$ seja quase-integrável. No entanto, pela Proposição $2.22 X$ é sela global. Este é um exemplo de sela global que não é um difeomorfismo local e tão pouco injetivo.

Exemplo 2.20. Outro exemplo de sela global com as mesmas características do caso anterior é $X(x, y)=\left(-x-x^{2}-2 x y-y^{2}, y+y^{2}-x^{2}\right)$.

Observação 2.21. Desde que o divergente de um campo quadrático $X=(f, g)$ denotado por:

$X(x, y)=\left(a_{33}+a_{13} x+a_{31} y+a_{11} x^{2}+a_{12} x y+a_{22} y^{2}, b_{33}+b_{13} x+b_{31} y+b_{11} x^{2}+b_{12} x y+b_{22} y^{2}\right)$,

é dado por $\operatorname{Div}(X)=\partial f / \partial x+\partial g / \partial y=a_{13}+b_{31}+\left(2 a_{11}+b_{12}\right) x+\left(a_{12}+2 b_{22}\right) y$. Temos que $X \in \mathfrak{D}^{1}\left(\mathbb{R}^{2}\right)$ se, e somente se, $b_{12}=-2 a_{11}$ e $a_{12}=-2 b_{22}$. Portanto, são poucos os campos quadráticos satisfazendo tal condição, a despeito da próxima proposição que afirma serem selas globais, todos os campos quadráticos em $\mathfrak{X}_{s}^{1}\left(\mathbb{R}^{2}\right)$.

Proposição 2.22. Se $X \in \mathfrak{X}_{s}^{1}\left(\mathbb{R}^{2}\right)$ é um campo polinomial quadrático, então $X$ é uma sela global.

Dem.: Nosso primeiro passo será obter uma mudança do sistema de coordenadas Oxy numa vizinhança de $O$ para outro no qual as separatrizes da sela $O$ estejam contidas nos novos eixos coordenados do plano. Pelo teorema da variedade estável e instável numa vizinhança $V$ de $O$ tais variedades invariantes podem ser definidas implicitamente por

$$
\sigma_{1}(x, y)=0, \text { e } \sigma_{2}(x, y)=0
$$


onde $\sigma_{i}, i=1,2$ tem a mesma classe de diferenciabilidade de $X$. Assim, definimos as novas coordenadas $(u, v)$ por

$$
u=\sigma_{1}(x, y), \text { e } v=\sigma_{2}(x, y)
$$

Deste modo teremos $u=0 \Leftrightarrow \sigma_{1}(x, y)=0$, ou seja, a variedade invariante definida por $\sigma_{1}(x, y)=0$ corresponderá ao eixo $O v$ no novo sistema de coordenadas. Analogamente, para $v=0$. A condição para que a mudança esteja bem definida é:

$$
\sigma_{1}(x, y) \cap \sigma_{2}(x, y)=\{O\} \text { e } \frac{J\left(\sigma_{1}, \sigma_{2}\right)}{J(x, y)} \neq 0 .
$$

Tomando a forma de Jordan de $X$ na vizinhança $V$ da sela hiperbólica $O$ podemos assumir que $X(x, y)=\left(\lambda_{1} x+f_{1}(x, y), \lambda_{2} y+g_{1}(x, y)\right)$, onde $\lambda_{1}<0$ e $\lambda_{2}>0$. Assim, assumimos que as variedades invariantes de $O$ em $V$ se expressam por

$$
\begin{gathered}
W^{s}(0): y=a_{2} x^{2}+\ldots+a_{n} x^{n}+\ldots \\
W^{u}(0): x=b_{2} y^{2}+\ldots+b_{n} y^{n}+\ldots,
\end{gathered}
$$

onde as respectivas expressões são convergentes na vizinhança $V$, a qual pode ser reduzida se necessário. Tomando o novo sistema de coordenadas dentro desta vizinhança definindo $\sigma_{1}(x, y)=y-a_{2} x^{2}-\ldots-a_{n} x^{n}-\ldots$ e $\sigma_{2}(x, y)=x-b_{2} y^{2}-\ldots-b_{n} y^{n}-\ldots$, vejamos que as condições (2.6) são satisfeitas. Note que a primeira, $\sigma_{1}(x, y) \cap \sigma_{2}(x, y)=\{O\}$, é trivialmente satisfeita e de

$$
\frac{J\left(\sigma_{1}, \sigma_{2}\right)}{J(x, y)}(0,0)=\left|\begin{array}{ll}
0 & 1 \\
1 & 0
\end{array}\right|=-1
$$

segue também a segunda condição em (2.6). Pois, da continuidade do Jacobiano, reduzindo $V$ se necessário, segue que $\frac{J\left(\sigma_{1}, \sigma_{2}\right)}{J(x, y)}(p) \neq 0$ em $V$. Portanto, nesta vizinhança podemos tomar o novo sistema de coordenadas $(u, v)$ no qual $W^{s}(O)=\{v=0\}$ e $W^{u}(O)=\{u=0\}$.

Pelo que vimos acima em $V$ temos que $v=0$ e $u=0$ são invariantes. Desde que o campo $X$ é polinomial, segue que no desenvolvimento em série do campo $X(u, v)=$ $(f(u, v), g(u, v))$ temos

$$
\begin{aligned}
& f(u, v)=\lambda_{1} u+a_{20} u^{2}+a_{11} u v+a_{02} v^{2}+\ldots \\
& g(u, v)=\lambda_{2} v+b_{20} u^{2}+b_{11} u v+b_{02} v^{2}+\ldots,
\end{aligned}
$$


de tal forma que $a_{02}, a_{03}, \ldots$ e $b_{20}, a_{30}, \ldots$ são nulos. Ou seja,

$$
\begin{aligned}
& f(u, v)=u\left(\lambda_{1}+a_{20} u+a_{11} v+a_{30} u^{2}+\ldots\right) \\
& g(u, v)=v\left(\lambda_{2}+b_{11} u+b_{02} v+b_{21} u^{2}+\ldots\right) .
\end{aligned}
$$

Assim a expressão local das variedades invariantes se converte em global.

Aplicando esta mudança no caso quadrático, voltando a notação habitual $(x, y)$, sem perda de generalidade, podemos considerar o sistema associado ao campo $X$ definido por

$$
\begin{aligned}
& x^{\prime}=f(x, y)=x\left(\lambda_{1}+a_{10} x+a_{01} y\right) \\
& y^{\prime}=g(x, y)=y\left(\lambda_{2}+b_{10} x+b_{01} y\right) .
\end{aligned}
$$

Para que $X \in \mathfrak{X}_{s}^{1}\left(\mathbb{R}^{2}\right)$ é necessário a unicidade de pontos singulares. Note que já temos $X(0,0)=(0,0)$. Além disto, substituindo $x=0$ na segunda equação de 2.7 e igualando a zero obtemos $y=0$ ou $y=-\lambda_{2} / b_{01}$, o que implica em dois pontos singulares a não ser que $b_{01}=0$. Analogamente, fazendo $y=0$ na primeira equação de 2.7$)$ e igualando a zero teremos $x=0$ ou $x=-\lambda_{1} / a_{10}$, isto implica que $X$ possuirá mais de um ponto singular se $a_{10} \neq 0$. Portanto, nosso sistema se reduz a $X(x, y)=\left(x\left(\lambda_{1}+a_{01} y\right), y\left(\lambda_{2}+b_{10} x\right)\right)$. Porém nesta forma ainda existirão mais de um ponto singular para $X$. Fazendo uma análise análoga à vista acima concluímos que as únicas possibilidades para $X$ são: a) $X(x, y)=(f(x, y), g(x, y))=\left(\lambda_{1} x, y\left(\lambda_{2}+b_{10} x\right)\right)$ e b) $X(x, y)=\left(x\left(\lambda_{1}+a_{01} y, \lambda_{2} y\right)\right.$.

Faremos agora um blow-up no infinito a fim de entender o que ocorre com estes campos próximo de $\infty$. Faremos isto no caso concreto a) sendo o b) inteiramente análogo. O primeiro passo é passar o sistema associado para coordenadas polares $x=r \cos \theta$ e $y=r \operatorname{sen} \theta$. Assim, o novo sistema fica:

$$
\begin{gathered}
\frac{d r}{d t}=\frac{1}{r}(r \cos \theta f(r, \theta)+r \operatorname{sen} \theta g(r, \theta))=\cos \theta f(r, \theta)+\operatorname{sen} \theta g(r, \theta) \\
\frac{d \theta}{d t}=\frac{1}{r^{2}}(r \cos \theta g(r, \theta)-r \operatorname{sen} \theta f(r, \theta))=\frac{1}{r}(\cos \theta g(r, \theta)-\operatorname{sen} \theta f(r, \theta)),
\end{gathered}
$$

ou seja,

$$
\begin{gathered}
\frac{d r}{d t}=r\left(\lambda_{1} \cos ^{2} \theta+\lambda_{2} \operatorname{sen}^{2} \theta+b_{10} r \cos \theta \operatorname{sen}^{2} \theta\right) \\
\frac{d \theta}{d t}=\cos \theta \operatorname{sen} \theta\left(-\lambda_{1}+\lambda_{2}+b_{10} r \cos \theta\right)
\end{gathered}
$$

Agora, pela mudança de coordenadas $r=1 / \rho$, levamos o infinito na origem. $\mathrm{O}$ 
sistema fica

$$
\begin{gathered}
\frac{d \rho}{d t}=\bar{f}(\rho, \theta)=\frac{d \rho}{d r} \frac{d r}{d t}=-\lambda_{1} \rho \cos ^{2} \theta-\lambda_{2} \rho \operatorname{sen}^{2} \theta-b_{10} \cos \theta \operatorname{sen}^{2} \theta \\
\frac{d \theta}{d t}=\bar{g}(\rho, \theta)=\cos \theta \operatorname{sen} \theta\left(-\lambda_{1}+\lambda_{2}+\frac{b_{10}}{\rho} \cos \theta\right) .
\end{gathered}
$$

Multiplicamos o sistema por $\rho$ a fim de que em $\rho=0$ ele seja invariante (isto é, $\frac{d \rho}{d t}=0$ ). Neste caso, obtemos um novo sistema satisfazendo

$$
\begin{gathered}
\frac{d \rho}{d t}(0, \theta)=0 \\
\frac{d \theta}{d t}(0, \theta)=b_{10} \cos ^{2} \theta \operatorname{sen} \theta .
\end{gathered}
$$

As raízes deste sistema são $\theta=0, \pi / 2, \pi, 3 \pi / 2$. Como não há outras raízes para $\rho=0$ não haverão setores pseudo-hiperbólicos no infinito e portanto, usando a notação das subseções anteriores, segue que as folheações $W_{i}$ serão triviais para $i=0,1,2,3$ e pelo Teorema 1.8 $X$ é uma sela global.

\subsubsection{Folheações do plano associadas a $X \in \mathfrak{X}_{s}^{1}\left(\mathbb{R}^{2}\right)$}

Dado $X \in \mathfrak{X}_{s}^{1}\left(\mathbb{R}^{2}\right)$, nos Teoremas A e B foram estabelecidas condições garantindo a trivialidade das folheações $\mathcal{F}\left(W_{i}\right)$, para todo $i=0, \ldots, 3$, o que equivale a dizer que $X$ é uma sela global. Por outro lado, vimos no Exemplo 2.1 que de fato isto nem sempre ocorre. Assim, uma questão que surge de maneira natural é a de estudar as possíveis folheações do plano realizadas como órbitas de campos vetoriais em $\mathfrak{X}_{s}^{1}\left(\mathbb{R}^{2}\right)$. Nesta seção será feita uma descrição parcial de tais folheações. De fato, daremos uma caracterização, por meio de um grafo, destas folheações restritas a um domínio do plano (cf. Teorema 2.30). Veremos que esse grafo é constituído de quatro árvores, cada uma representando um domínio contido em $W_{i}, i=0, \ldots, 3$. A fim de definir tal domínio e descrever esta caracterização parcial, lançaremos mão do conceito de campos vetoriais em rotação, o qual passamos a descrever.

Dado $X \in \mathfrak{X}^{1}\left(\mathbb{R}^{2}\right)$, seja $F(\theta)=\left\{X_{\theta} ; \theta \in I\right\}$, onde $I:=[0, \pi]$, a família de campos vetoriais em rotação definida por

$$
X_{\theta}=(f \cos \theta-g \sin \theta, f \sin \theta+g \cos \theta) .
$$


O conceito de família de campos vetoriais em rotação foi introduzido por Duff [18] num contexto mais geral. Este caso é um exemplo do que Duff chamou de família uniforme completa de campos vetoriais em rotação. Por definição, em tais famílias os pontos de equilíbrio dos campos vetoriais $X_{\theta}$ não variam em relação a $\theta$. Além disto, Duff provou em [18] que selas hiperbólicas persistem a rotações.

Pelas considerações acima, temos que o conjunto $\mathfrak{X}_{s}^{1}\left(\mathbb{R}^{2}\right)$ é invariante por rotações, isto é, dado $X \in \mathfrak{X}_{s}^{1}\left(\mathbb{R}^{2}\right)$ temos que para todo $\theta \in I$ o campo vetorial em rotação $X_{\theta}$ pertence também a $\mathfrak{X}_{s}^{1}\left(\mathbb{R}^{2}\right)$. Logo, como consequência do Teorema de Poincaré-Bendixon, o comportamento das órbitas de $X_{\theta}$ é idêntico ao das órbitas de $X$, ou seja, não há órbitas fechadas associadas a este campo e $\omega\left(\gamma_{\theta}\right)=\alpha\left(\gamma_{\theta}\right)=\varnothing$ para cada trajetória $\gamma_{\theta}$ de $X_{\theta}$, exceto para a sela $O$ e suas separatrizes. Em particular, podemos ordenar as quatro separatrizes de $O$ como fizemos com as separatrizes $s_{i}, i=0, \ldots, 3$, da sela $O$ de $X$. Assim, dado $\theta \in I$ vamos denotar por $l_{\theta}^{i}$ a separatriz de $O$ do campo $X_{\theta}$ tal que $l_{0}^{i}=s_{i}$. Note que pela definição da família $F(\theta)$ temos que $X_{\pi}=-X$ e portanto a separatriz $l_{\pi}^{i}$ coincide a menos de orientação com $s_{j}=l_{0}^{j}$, onde $j=(i+1) \bmod 4$.

O próximo resultado fornece outras propriedades interessantes da família $F(\theta)$ quando $X \in \mathfrak{X}_{s}^{1}\left(\mathbb{R}^{2}\right)$.

Lema 2.23. Seja $X \in \mathfrak{X}_{s}^{1}\left(\mathbb{R}^{2}\right)$ e $F(\theta)=\left\{X_{\theta} ; \theta \in I\right\}$ a familia de campos vetoriais em rotação definida em (2.8). Então, dado $\theta \in(0, \pi)$, valem as seguintes afirmações:

1) Se $\gamma$ e $\gamma_{\theta}$ são órbitas de $X$ e $X_{\theta}$, respectivamente, então $\#\left(\gamma \cap \gamma_{\theta}\right) \leq 1$;

2) Se $s_{i}$ e $l_{\theta}^{j}$ são separatrizes da sela $O$ de $X$ e $X_{\theta}$, respectivamente, então $s_{i} \cap l_{\theta}^{j}=\varnothing$, para quaisquer $i, j=0, \ldots, 3$.

Dem.: Para provar 1), observe que fixado $\theta \in(0, \pi)$ o ângulo entre $X_{\theta}$ e $X$ é constante igual a $\theta \neq 0$ em $\mathbb{R}^{2} \backslash\{0\}$ e portanto as trajetórias de $X_{\theta}$ intersectam as trajetórias de $X$ transversalmente.

Suponha, por absurdo, que existem órbitas $\gamma_{\theta}$ e $\gamma$ de $X_{\theta}$ e $X$, respectivamente, que se intersectam em sucessivos pontos $p_{1}, p_{2}, p_{3}, \ldots \in \gamma_{\theta} \cap \gamma$. Denote por $\left[p_{1}, p_{2}\right]_{\theta}$ e $\left[p_{1}, p_{2}\right]$ os arcos das trajetórias $\gamma_{\theta}$ e $\gamma$, respectivamente, entre os pontos $p_{1}$ e $p_{2}$. Seja $K$ a região compacta limitada pela união destes arcos. Desde que as órbitas de $X_{\theta}$ intersectam $\left[p_{1}, p_{2}\right]$ com o mesmo ângulo $\theta$, para cada ponto $p \in\left[p_{1}, p_{2}\right]$ existe uma única órbita $\gamma_{\theta}(p)$ de $X_{\theta}$ passando por $p$ entrando no compacto $K$. Portanto $K$ é positivamente invariante em relação a $X_{\theta}$ o que implica na existência de um ponto singular no interior deste compacto. Por outro lado, elegendo outro par de pontos sucessivos em $\gamma_{\theta} \cap \gamma$, caso necessário, pode-se assumir que $K$ não contém a sela $O$. Tal contradição prova o item 1). 
O item 2) segue de 1). De fato, se uma separatriz $l_{\theta}^{j}$ da sela de $X_{\theta}$ intersecta alguma separatriz $s_{i}$ da sela $O$ de $X$, então existe uma região compacta limitada pela origem $O$ e os segmentos de órbitas adjacentes de $l_{\theta}^{j}$ e $s_{i}$. Como $X_{\theta}$ não possui outros pontos de equilíbrio a não ser $O$ e pelo Teorema de Hartman-Grobman só as separatrizes de $O$ podem se acumular neste ponto singular, as trajetórias de $X_{\theta}$ que entram em tal compacto obrigatoriamente devem deixá-lo e portanto intersectam pelo menos duas vezes a órbita $s_{i}$ de $X$, contradizendo o item 1).

Em [48], Perko provou o seguinte resultado sobre o movimento de separatrizes de selas hiperbólicas em rotação no caso em que $X$ é analítico. Adaptamos aqui seus argumentos para o caso em que o campo $X$ é de classe $C^{1}$ :

Lema 2.24. Sejam $X: \mathbb{R}^{2} \rightarrow \mathbb{R}^{2}$ um campo vetorial de classe $C^{1}$ com uma sela hiperbólica em $O$ e $F(\theta)$ a família de campos em rotação definida em (2.8). Então cada separatriz $l_{\theta}^{i}$ da sela $O$ associada a $X_{\theta}$ gira monotonicamente no sentido anti-horário em cada círculo suficientemente pequeno centrado em $O$, de sua posição inicial até sua posição final $l_{\pi}^{i}=l_{0}^{j}$, onde $j=(i+1) \bmod 4$, quando $\theta$ varia de 0 a $\pi$.

Dem.: Suponha, por absurdo, que para algum $i \in\{0,1,2,3\}$, não exista um círculo tal que a interseção da separatriz $l_{\theta}^{i}$ com tal círculo se movimente monotonicamente no sentido anti-horário. Neste caso, podemos tomar $\theta_{1}<\theta_{2}$ em $[0, \pi]$ e um ponto $p_{0} \in l_{\theta_{1}}^{i}$ tais que a solução $\gamma_{\theta_{2}}$ de $X_{\theta_{2}}$ intersectando a separatriz $l_{\theta_{1}}^{i}$ de $X_{\theta_{1}}$ no ponto $p_{0}$, é tal que o ângulo entre $\frac{d \gamma_{\theta_{2}}}{d t}\left(t_{2}\right)=X_{\theta_{2}}\left(p_{0}\right)$ e $\frac{d l_{\theta_{1}}^{i}}{d t}\left(t_{1}\right)=X_{\theta_{1}}\left(p_{0}\right)$ é próximo de $\theta_{1}-\theta_{2}<0$, onde $\gamma_{\theta_{2}}\left(t_{2}\right)=p_{0}$ e $l_{\theta_{1}}^{i}\left(t_{1}\right)=p_{0}$. De fato, podemos fazer tal ângulo tão próximo de $\theta_{1}-\theta_{2}$ quanto queiramos, bastando para isto tomar $p_{0} \in l_{\theta_{1}}^{i}$ suficientemente próximo da origem $O$ (cf. Figura 2.7).

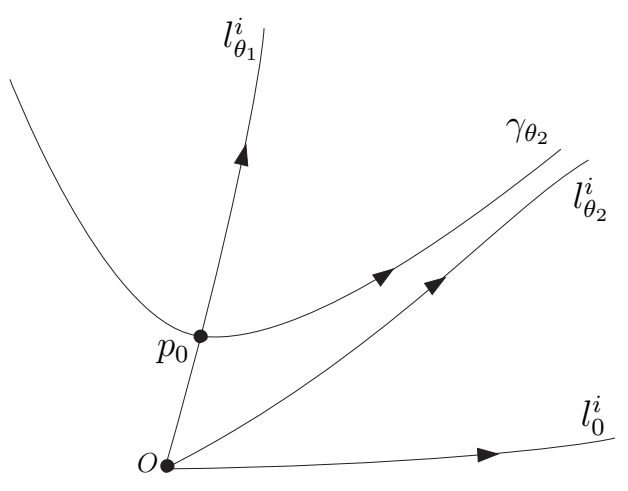

Figura 2.7: Lema 2.24 
Porém, pela definição da família $F(\theta)$, para todo ponto $p \in \mathbb{R}^{2} \backslash\{O\}$ o ângulo entre os vetores $X_{\theta_{2}}(p)$ e $X_{\theta_{1}}(p)$ é igual a $\theta_{2}-\theta_{1}>0$. Esta contradição mostra o lema.

Este resultado nos permite concluir que as separatrizes de $O$ em rotação cobrem uma bola de $\mathbb{R}^{2}$ suficientemente pequena centrada na origem. É claro que este comportamento não é necessariamente global, ou seja, não é verdade que cada separatriz $l_{\theta}^{i}$ da sela $O$ associada a $X_{\theta}$ se move monotonicamente no sentido positivo num círculo qualquer centrado na origem do plano. Portanto, não é possível concluir que, em geral, o conjunto das quatro separatrizes em rotação de $X$ cubra todo $\mathbb{R}^{2}$, quando $\theta$ varia de 0 a $\pi$.

Observação 2.25. As considerações do parágrafo anterior motiva a introdução das seguintes noções: dado $i \in\{0, \ldots, 3\}$, seja $L_{i}$ o domínio conexo em $W_{i}$ dado pela família $\left\{l_{\theta}^{i} ; \theta \in \stackrel{\circ}{I}\right\}$. Denote por $G_{i}$ a união das regiões canônicas de $\mathcal{F}\left(W_{i}\right)$ contidas em $L_{i}$. Isto decompõe cada folheação $\mathcal{F}\left(W_{i}\right)$ em duas subfolheações: a folheação $\mathcal{F}\left(G_{i}\right)$ em $G_{i}$ e a folheação no complemento $W_{i} \backslash G_{i}$.

No que segue, apresentamos uma caracterização da subfolheação $\mathcal{F}\left(G_{i}\right)$. Isto fornece uma classificação somente parcial das folheações do plano induzidas por campos vetoriais em $\mathfrak{X}_{s}^{1}\left(\mathbb{R}^{2}\right)$, uma vez que as subfolheações nas regiões $W_{i} \backslash G_{i}$ não apresentarão restrições. Como será útil, para caracterizar a folheação $\mathcal{F}\left(G_{i}\right)$, passamos a introduzir uma classe de setores pseudo-hiperbólicos no infinito, que está estreitamente associada à noção de níveis das regiões canônicas (cf. Definição 2.3) das folheações associadas a campos vetoriais em $\mathfrak{X}_{s}^{1}\left(\mathbb{R}^{2}\right)$.

Definição 2.26. [Setor pseudo-hiperbólico inicial no infinito] Dado $X \in \mathfrak{X}_{s}^{1}\left(\mathbb{R}^{2}\right)$. Um setor pseudo-hiperbólico no infinito $S$ de $X$, como na Definição 2.5, é dito inicial se uma das semi-trajetórias não limitadas $\left[q_{1}, \infty\right)$ ou $\left(\infty, q_{2}\right]$ de $X$ estiver contida em uma separatriz inicial da região canônica que contém $S$ (veja Figura 2.8).

Neste caso, será chamada região pseudo-hiperbólica inicial associada $S$ a região não limitada dada por

$$
S_{h}=\bigcup_{q \in\left(p_{1}, q_{1}\right)} \gamma_{q}
$$

Observação 2.27. Na Figura 2.3 à esquerda existem três setores pseudo-hiperbólicos iniciais no infinito enquanto que à direita tais setores não ocorrem.

Na demonstração do próximo lema, as notações da Definição 2.26 e da Figura 2.8 serão seguidas. 


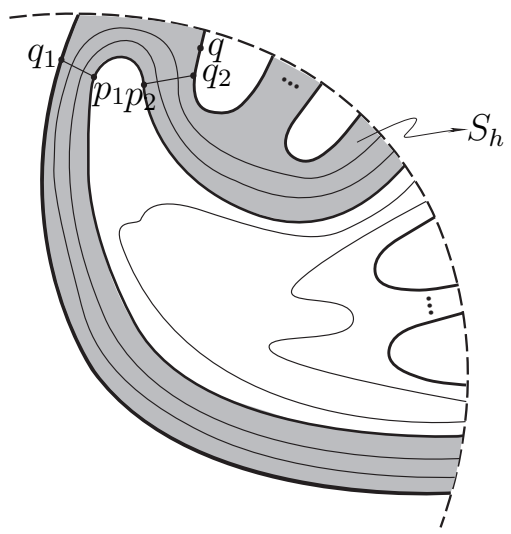

Figura 2.8: Setor pseudo-hiperbólico inicial no infinito

Lema 2.28. Se $X \in \mathfrak{X}_{s}^{1}\left(\mathbb{R}^{2}\right)$ e $C$ é uma região canônica contida de $G_{i}, i=0, \ldots, 3$, então $C$ não possui setor pseudo-hiperbólico inicial no infinito.

Dem.: Suponha, por contradição, que $X$ possua um setor pseudo-hiperbólico inicial $S$ no infinito contido na região canônica $C \subset G_{i}$, para algum $i \in\{0,1,2,3\}$. Sem perda de generalidade, considere que $\left(\infty, q_{1}\right]$ é a semi-trajetória não limitada contida na separatriz inicial de $C$.

Tome um ponto arbitrário em $q \in\left[q_{2}, \infty\right)$. Desde que $C \subset G_{i}$, existe $\theta \in(0, \pi)$ tal que uma separatriz $l_{\theta}^{i}$ de $X_{\theta}$ passa pelo ponto $q$. Denote por $l_{\theta}^{i}(t)$ a solução da equação diferencial associada a $X_{\theta}$ tal que $l_{\theta}^{i}(0)=q$, e assuma, sem perda de generalidade, que tal separatriz seja estável.

Para concluir o lema basta mostrar que $l_{\theta}^{i}$ intersecta duas vezes alguma órbita de $X$, pois isto contradiz o item 1) do Lema 2.23 .

Observe que para $t>0$ suficientemente grande, a solução $l_{\theta}^{i}(t)$ necessariamente entra na região pseudo-hiperbólica inicial $S_{h}$ associada a $S$. Logo, existe um ponto $\bar{p} \in\left[p_{2}, q_{2}\right]^{*}$ tal que para cada $p \in\left[\bar{p}, q_{2}\right]^{*}$ existe $t \in \mathbb{R}$ satisfazendo $l_{\theta}^{i}(t) \cap \gamma_{p} \neq \varnothing$, onde $\gamma_{p}$ é a órbita de $X$ passando pelo ponto $p$. Além disto, desde que $l_{\theta}^{i}(t) \rightarrow O$ quando $t \rightarrow \infty$ temos que $l_{\theta}^{i}(t)$ necessariamente sai de $S_{h}$ e isto implica que $l_{\theta}^{i}(t)$ intersectará todas as órbitas $\gamma_{p}$ de $X$, com $p \in\left[\bar{p}, q_{2}\right]^{*}$, em outras palavras $l_{\theta}^{i}(t)$ intersecta tais órbitas duas vezes.

Pelo Lema 2.28 e da classificação das regiões canônicas das folheações no plano, concluímos que na subfolheação $\mathcal{F}\left(G_{i}\right)$ as regiões canônicas são do tipo (I), (III) ou (IV), com a restrição adicional que adjacente à separatriz inicial haverão sempre setores parabólicos no infinito (veja Figura 2.9). As regiões canônicas do tipo (III) e (IV) satisfazendo tal 
restrição serão denotadas por $(\overline{\mathrm{III}})$ e $(\overline{\mathrm{IV}})$. Ou seja, temos o seguinte:

Corolário 2.29. Dado $X \in \mathfrak{X}_{s}^{1}\left(\mathbb{R}^{2}\right)$, toda região canônica em $G_{i}, i=0, \ldots, 3$, é do tipo (I), ( $\overline{\mathrm{III}})$ ou $(\overline{\mathrm{IV}})$.

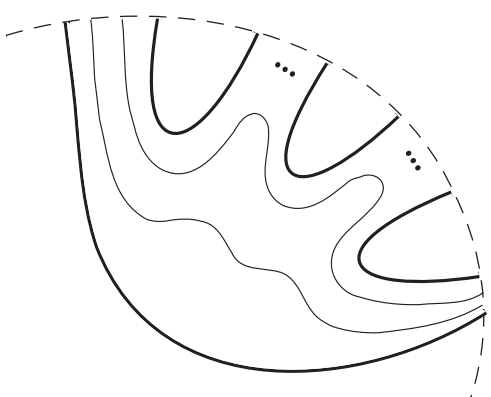

Figura 2.9: Região canônica do tipo (IV)

Note que uma região canônica do tipo (I) da subfolheação $\mathcal{F}\left(G_{i}\right)$ pode ser do tipo (V) se considerada com região canônica da folheação $\mathcal{F}\left(W_{i}\right)$ em todo $W_{i}$. Portanto, uma região canônica de $\mathcal{F}\left(G_{i}\right)$ que não é do tipo (I) possui, além da separatriz inicial, pelo menos mais duas separatrizes. Assim, a subfolheação $\mathcal{F}\left(G_{i}\right)$ em $G_{i}$, quando não for trivial, será um conjunto de regiões canônicas encaixadas do tipo (III) ou $(\overline{\mathrm{IV}})$ finalizando com regiões canônicas do tipo (I). Tal estrutura esta descrita no próximo teorema por meio de uma árvore.

Para melhor compreensão do enunciado do próximo resultado serão apresentadas a seguir as definições de alguns elementos básicos da teoria de grafos. Um grafo é uma estrutura $G(V, A)$ onde $V$ é um conjunto não vazio de objetos denominados vértices e $A$ é um conjunto de pares não ordenados de $V$ chamados arestas. Um grafo dirigido consiste de um conjunto $V$ de vértices, um conjunto $E$ de arestas e aplicações $s, t: E \rightarrow V$, onde $s(e)$ é a fonte e $t(e)$ é o alvo da aresta direcionada. Em um grafo dirigido, define-se como grau de saída (resp. grau de entrada) de um vértice o número de arestas saindo do mesmo (resp. entrando no mesmo). Ciclo (ou circuito) é um caminho de arestas que começa e termina num mesmo vértice. Uma árvore é um grafo conexo (existe caminho entre quaisquer dois de seus vértices) e acíclico (não possui ciclos).

Teorema 2.30. Dado $X \in \mathfrak{X}_{s}^{1}\left(\mathbb{R}^{2}\right)$, para cada folheação $\mathcal{F}\left(G_{i}\right)$ sem separatrizes limite, $i \in\{0, \ldots, 3\}$, existe uma árvore associada tal que o conjunto ordenado destas árvores é um invariante completo para o sistema restrito a $\cup_{i=0}^{3} G_{i}$ (a menos de equivalência topológica). Cada árvore é dirigida e satisfaz:

1) possui uma única fonte; 
2) o grau de saída de cada vértice é distinto de 1.

Dem.: As respectivas árvores são construídas da seguinte forma: cada vértice representa uma região canônica, uma aresta entre dois vértices representa uma separatriz em comum entre as respectivas regiões. Cada árvore é dirigida de tal forma que o vértice correspondente à região canônica inicial seja sua fonte. Se uma região canônica em $\mathcal{F}\left(G_{i}\right)$ é do tipo (I) então seu vértice tem grau de saída igual a 0. Se não for do tipo (I) então, pelo Corolário 2.29, terá pelo menos duas arestas saindo do seu vértice.

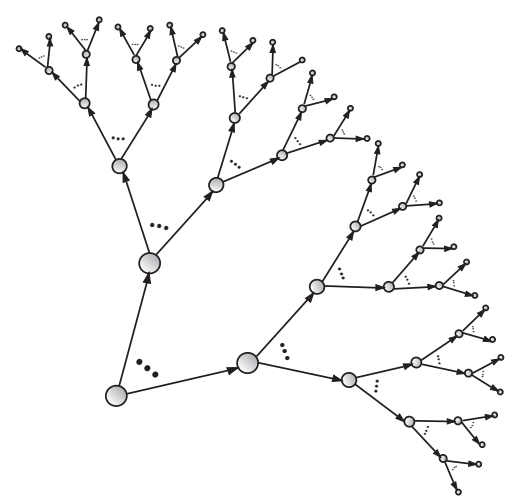

Figura 2.10: Árvore de $\mathcal{F}\left(G_{i}\right)$

Observação 2.31. Ressaltamos que a subfolheação $\mathcal{F}\left(G_{i}\right)$ pode ser caracterizada por meio de outros diagramas seguindo, por exemplo, [37] ou [60]. O que apresentamos no Teorema 2.30 é um invariante mais simples que o invariante no caso geral, uma vez que não é necessário distinguir a árvore. Mais precisamente, vimos que para um campo vetorial em $\mathfrak{X}_{s}^{1}\left(\mathbb{R}^{2}\right)$ o conjunto das regiões canônicas em $G_{i}$ sempre têm uma estrutura de árvore. Em geral, para descrever uma folheação do plano são necessárias informações adicionais sobre os tipos de regiões canônicas. Por exemplo, as duas folheações da Figura 2.3 têm a mesma árvore associada, mas obviamente elas não são topologicamente equivalentes. Árvores distinguidas como definidas, por exemplo, em [60] dão tais informações adicionais.

Observação 2.32. A Figura 2.11 ilustra que nem sempre a família de separatrizes rotacionadas $\left\{l_{\theta}^{i} ; \theta \in(0, \pi)\right\}$ cobre a região $W_{i}$. Neste caso, existirá pelo menos uma região canônica que não está contida em $G_{i}$, na qual podem existir setores pseudo-hiperbólicos iniciais (veja esquerda da Figura 2.11).

Portanto seria interessante encontrar condições garantindo que a família de separatrizes em rotação $\left\{l_{\theta}^{i} ; \theta \in(0, \pi)\right\}$ cubra o aberto $W_{i}$, para todo $i=0, \ldots, 3$. Isto 

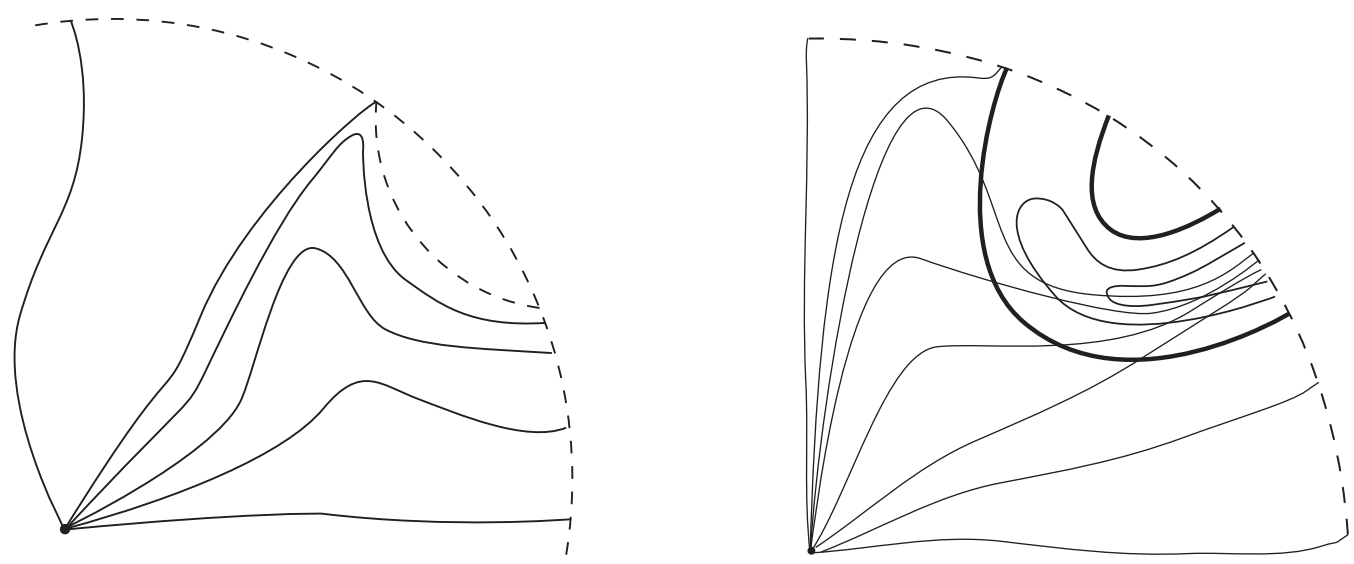

Figura 2.11: Ilustração da Observação 2.32

determinaria uma classe de campos em $\mathfrak{X}_{s}^{1}\left(\mathbb{R}^{2}\right)$ na qual a caracterização das folheações $\mathcal{F}\left(W_{i}\right)$, dada no Teorema 2.30 , seria completa.

\subsection{Caso discreto}

Considere um difeomorfismo $f: \mathbb{R}^{2} \rightarrow \mathbb{R}^{2}$, de classe $C^{1}$, com um único ponto fixo, na origem $O$ de $\mathbb{R}^{2}$, do tipo sela hiperbólica. Vamos denotar por $\operatorname{Diff}_{s}\left(\mathbb{R}^{2}\right)$ o conjunto de tais difeomorfismos e por Diff $f_{s}^{+}\left(\mathbb{R}^{2}\right)$ a interseção de $\operatorname{Diff} f_{s}\left(\mathbb{R}^{2}\right)$ com o conjunto de difeomorfismos do plano que preservam orientação.

Como já visto na Seção 1.3 , dado $f \in \operatorname{Diff}_{s}\left(\mathbb{R}^{2}\right)$ denotaremos por $W^{s}(O)$ e $W^{u}(O)$, respectivamente, as variedades estável e instável de $O$. Chamamos de separatrizes do ponto fixo $O$ as componentes conexas de $W^{u}(O) \cup W^{s}(O) \backslash\{O\}$, e as denotamos por $s_{0}, \ldots, s_{3}$, onde a ordem é dada de acordo com o sentido crescente do ângulo de sua tangente no ponto $O$ com o semi-eixo positivo $x$.

Definição 2.33. Dizemos que uma separatriz estável (resp. instável) $s_{i}$ de $O$ se acumula somente no $\infty$ se $\omega\left(s_{i}\right)=\bigcup_{p \in s_{i}} \omega(p)=\{O\}$ e $\alpha\left(s_{i}\right)=\bigcup_{p \in s_{i}} \alpha(p)=\varnothing$ (resp. $\omega\left(s_{i}\right)=\varnothing e$ $\left.\alpha\left(s_{i}\right)=\{O\}\right)$.

No contexto de sistemas dinâmicos discretos não foi possível estabelecer uma equivalência com alguma sela linear. Com efeito, o que se obteve, nos Teoremas C e D, foi que sob certas condições um difeomorfismo $f \in \operatorname{Diff}_{s}\left(\mathbb{R}^{2}\right)$ tem o mesmo comportamento assintótico de uma sela linear, isto é, as quatro separatrizes $\left\{s_{0}, \ldots, s_{3}\right\}$ de $O$ se acumulem somente no infinito e todo ponto $p \in \mathbb{R}^{2} \backslash\left\{O \cup s_{0} \cup \ldots \cup s_{3}\right\}$ satisfaz $\omega(p)=\alpha(p)=\varnothing$. Neste 
caso diremos que $f \in \mathcal{S} \subset \operatorname{Diff}_{s}\left(\mathbb{R}^{2}\right)$ quando satisfizer tais propriedades. A fim de obter esses resultados assumiremos que uma das separatrizes $s_{i}$ da sela $O$ de $f \in \operatorname{Diff}_{s}\left(\mathbb{R}^{2}\right)$ se acumula somente no infinito. Neste caso, como consequência do Teorema 2.35, não haverão interseções transversais entre as variedades estável e instável de $O$, sempre que $s_{i}$ for $f$-invariante.

Observação 2.34. Se $f \in \operatorname{Diff}_{s}^{+}\left(\mathbb{R}^{2}\right)$, então pelo Teorema de Hartman-Grobman e as propriedades das variedades invariantes de $O$, segue que $f$ satisfaz uma das seguintes possibilidades: $f\left(s_{i}\right)=s_{i}$, para todo $i=0, \ldots, 3$, ou $f\left(s_{1}\right)=s_{3}, f\left(s_{3}\right)=s_{1}, f\left(s_{0}\right)=s_{2}$ e $f\left(s_{2}\right)=s_{0}$. Em outras palavras, se $f \in D i f f_{s}^{+}\left(\mathbb{R}^{2}\right)$ então as quatro separatrizes são $f$-invariantes ou nenhuma delas é $f$-invariante.

Seja $\gamma:[0, \infty) \rightarrow \mathbb{R}^{2}$ um mergulho topológico de $[0, \infty)$. Identificamos a curva $\gamma$ com sua imagem $\gamma([0, \infty))$. Dado um difeomorfismo $f: \mathbb{R}^{2} \rightarrow \mathbb{R}^{2}$, dizemos que $\gamma$ é um raio $f$-invariante se $\gamma(0)=O, f(\gamma) \subset \gamma$ e $|\gamma(t)| \rightarrow \infty$ quando $t \rightarrow \infty$.

O seguinte resultado provado em [2] jogará um papel fundamental em nossos resultados, nele denotamos por $\operatorname{Fix}(f)$ o conjunto dos pontos fixos de $f$. Em certo sentido, este teorema é o análogo, no âmbito discreto, ao Teorema de Poincaré-Bendixon no contexto de campos vetoriais.

Teorema 2.35. (Alarcón, Guínez e Gutierrez [2]) Seja $f: \mathbb{R}^{2} \rightarrow \mathbb{R}^{2}$ uma aplicação contínua, injetiva e que preserva orientação. Se $\operatorname{Fix}(f)=\{O\}$ e existe um raio $f$-invariante $\gamma$, então $\Omega(f) \subset \gamma$ e $\omega(p) \subset\{O\}$, para todo $p \in \mathbb{R}^{2}$.

Como os interesses deste texto se restringem ao caso de $f$ ser um difeomorfismo do plano, este teorema nos permite obter conclusões análogas para o conjunto $\alpha$-limite.

Note que dado um difeomorfismo $f \in D i f f_{s}^{+}\left(\mathbb{R}^{2}\right)$ com uma separatriz $s_{i} f$-invariante se acumulando somente no infinito então $s_{i} \cup\{O\}$ constitui um raio $f$-invariante. No próximo resultado damos condições sob as quais concluímos, aplicando o Teorema 2.35. que as outras três separatrizes também se acumulam somente no infinito.

Teorema C. Dado $f \in$ Diff $_{s}^{+}\left(\mathbb{R}^{2}\right)$, se uma das separatrizes de $O$ define um raio $f$-invariante e não existem tangências entre as separatrizes. Então $f \in \mathcal{S}$.

Dem.: Seja $s_{1}$ a separatriz que define o raio $f$-invariante $\gamma$. Desde que $s_{1}$ é $f$-invariante pela Observação 2.34 as outras três separatrizes são também $f$-invariantes. Dada uma separatriz $s \neq s_{1}$ de $O$, para fixar os argumentos, considere que $s$ seja uma separatriz instável, o caso em que $s$ é estável é análogo. Assim, $\alpha(s)=\{O\}$ e, pelo Teorema 2.35. temos que $\omega(s) \subset\{O\}$. 
Portanto, se $s$ não se acumula somente no infinito, por hipótese, $s$ coincide com uma separatriz estável e $\omega(s)=\{O\}$. No que segue provaremos que tal coincidência não ocorre.

De fato, se $s$ é também uma separatriz estável então $s \cup O$ é uma curva fechada $f$-invariante em $\mathbb{R}^{2}$ que borda um subconjunto compacto $K$ de $\mathbb{R}^{2}$. Desde que $f$ é um difeomorfismo do plano, temos que $K$ é um subconjunto $f$-invariante de $\mathbb{R}^{2}$. Dado um ponto $p$ no interior de $K$, pelo Teorema 2.35, os iterados de $p$ só podem acumular na origem $O$ o que contradiz o Teorema de Hartman-Grobman.

No caso em que o difeomorfismo $f \in \operatorname{Diff}_{s}\left(\mathbb{R}^{2}\right)$ não preserva orientação necessariamente, vamos considerar a hipótese adicional que é a unicidade de órbitas 2-periódicas de $f$, ou de maneira equivalente, $\operatorname{Fix}\left(f^{2}\right)=\{O\}$. Neste caso veremos que a $f$-invariância da separatriz que acumula somente no infinito não será necessária.

Teorema D. Se $f \in \operatorname{Diff}_{s}\left(\mathbb{R}^{2}\right)$ possui uma separatriz definindo um raio $f^{2}$-invariante, as separatrizes não se tangenciam e $\operatorname{Fix}\left(f^{2}\right)=\{O\}$, então $f \in \mathcal{S}$.

Dem.: Note que mesmo quando $f \in \operatorname{Diff}_{s}\left(\mathbb{R}^{2}\right)$ for um difeomorfismo que reverte orientação, temos que $f^{2}$ é um difeomorfismo que preserva orientação. Além disto, por hipótese, $f^{2}$ possui uma separatriz $s_{i}$ definindo um raio $f^{2}$-invariante e $\operatorname{Fix}\left(f^{2}\right)=\{O\}$. Isto implica que o difeomorfismo $f^{2}$ pertence a $D i f f_{s}^{+}\left(\mathbb{R}^{2}\right)$ e, portanto, $f^{2}$ satisfaz todas as hipóteses do Teorema C. Logo, podemos concluir que as quatro separatrizes de $f^{2}$ e, consequentemente, de $f$ também acumulam somente no infinito. Além do mais, qualquer ponto $p \in \mathbb{R}^{2} \backslash\left\{W^{s}(O) \cup W^{u}(O)\right\}$ tem conjuntos limites $\omega(p)$ e $\alpha(p)$ vazios tanto em relação a $f^{2}$ quanto a $f$. Ou seja, $f \in \mathcal{S}$.

O próximo exemplo ilustra que dado um difeomorfismo $f \in \operatorname{Diff}_{s}^{+}\left(\mathbb{R}^{2}\right)$ nem sempre as quatro separatrizes de $O$ acumulam somente no infinito. Isto mostra a necessidade da $f$-invariância da separatriz acumulando somente no infinito no Teorema C.

Exemplo 2.36. Seja $f: \mathbb{R}^{2} \rightarrow \mathbb{R}^{2}$ definida por $f(x, y)=\left(f_{1}(x), f_{2}(y)\right)=(-x / 2,-2 y+$ $\left.g_{\epsilon}(y)\right)$ onde, para cada $0<\epsilon<1, g_{\epsilon}: \mathbb{R} \rightarrow \mathbb{R}$ é uma função de classe $C^{1}$ satisfazendo:

1) $0<g_{\epsilon}^{\prime}(y)<2$ para todo $y \in \mathbb{R}$ e $g_{\epsilon}^{\prime}(0)<1$;

2) $g_{\epsilon}$ é ímpar;

3) existe um positivo $y_{0} \in \mathbb{R}$ tal que $|y| \geq y_{0}$ implica $g_{\epsilon}^{\prime}(y)=2-\epsilon$.

Inicialmente mostraremos que 
(a) $f \in \operatorname{Diff} f_{s}^{+}\left(\mathbb{R}^{2}\right)$.

De fato, o item 1) implica a injetividade de $f$, uma vez que $f_{1}$ e $f_{2}$ são funções estritamente monótonas de uma variável. Desde que

$$
D f(x, y)=\left(\begin{array}{cc}
-1 / 2 & 0 \\
0 & -2+g_{\epsilon}^{\prime}(y)
\end{array}\right)
$$

por 1) temos que $\operatorname{det}(D f(x, y))=1-1 / 2 g_{\epsilon}^{\prime}(y)>0, \forall(x, y) \in \mathbb{R}^{2}$. Portanto, $f$ é um difeomorfismo do plano nele mesmo que preserva orientação.

Pela definição de $f$, o conjunto $\operatorname{Fix}(f)$ está contido no eixo $y$. Logo, a condição para existência de pontos $f$-fixos é equivalente a $f_{2}(y)=-2 y+g_{\epsilon}(y)=y$, ou seja, $g_{\epsilon}(y)=3 y$. Porém, pela restrição 1$)$, temos que $g_{\epsilon}(y) \neq 3 y$, para todo $y \in \mathbb{R}$, e assim segue que $\operatorname{Fix}(f)=\{O\}$. Além do mais, a condição $g_{\epsilon}^{\prime}(0)<1$ garante que tal ponto fixo é uma sela hiperbólica. Isto prova a afirmação (a).

Agora, afirmamos que

(b) $f$ tem uma órbita 2-periódica $\{(0, \bar{y}),(0,-\bar{y})\}$, para algum $\bar{y}>0$, contida no eixo $y$.

Antes de provar tal afirmação, note que $W^{s}(O)=s_{0} \cup O \cup s_{2}$ é o eixo $x$ e portanto as separatrizes $s_{0}$ e $s_{2}$ acumulam somente no infinito. Por outro lado, a afirmação (b) implica que apesar de $W^{u}(O)=s_{1} \cup O \cup s_{3}$ estar contida no eixo $y$, tal variedade invariante não é igual ao eixo $y$. De fato, $\omega\left(s_{1}\right)=(0, \bar{y})$ e $\omega\left(s_{3}\right)=(0,-\bar{y})$.

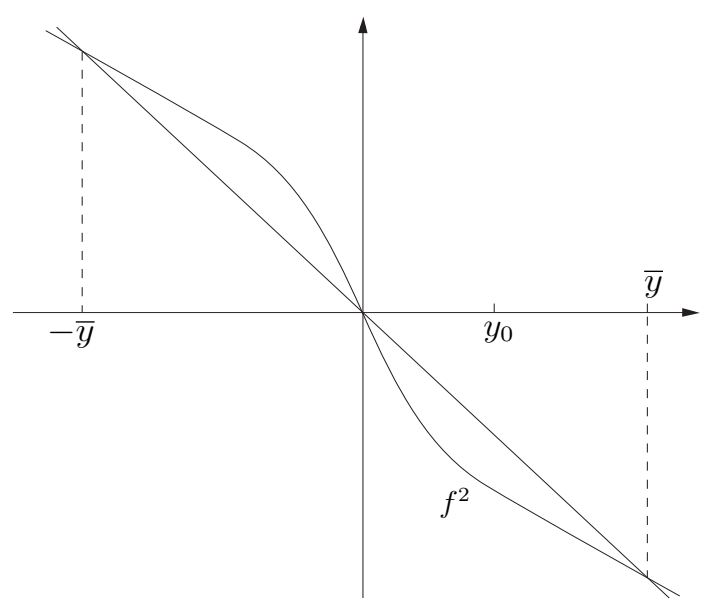

Figura 2.12 
Para provar (b), observe que os eixos $x$ e y são $f$-invariantes e que $f_{2}$ é simétrica com respeito a origem desde que é a soma de duas funções com tal propriedade. Assim, para garantir a existência de uma órbita 2-periódica de $f$ é suficiente garantir a existência de um ponto de interseção $\bar{y}(>0)$ entre os gráficos das funções $f_{2}$ e $h(y)=-y$, distinto da origem $O$, pois isto significa que $f_{2}(\bar{y})=h(\bar{y})=-\bar{y}$. A simetria de $f_{2}$ com respeito a origem, nos dá que $f(0, \bar{y})=(0,-\bar{y})$ e $f(0,-\bar{y})=(0, \bar{y})$. A existência do ponto de interseção $\bar{y}$ é garantida pelo fato da propriedade $0<g_{\epsilon}^{\prime}(0)<1$ implicar $-2<f_{2}^{\prime}(0)<-1$ e da condição 3) garantir que se $|y| \geq y_{0}>0$ então $f_{2}^{\prime}(y)=-2+g_{\epsilon}^{\prime}(y)=-\epsilon>-1$. Veja Figura 2.12.

Uma função $g_{\epsilon}$ em concreto satisfazendo as condições do exemplo 2.36 para $y_{0}=1$ é dada por:

$$
g_{\epsilon}(y)=\left\{\begin{array}{cll}
\frac{(2-\epsilon) y^{3}}{1+y^{2}} & \text { if } & |y| \leq 1 \\
(2-\epsilon) y-1+\epsilon / 2 & \text { if } & y>1 \\
(2-\epsilon) y+1-\epsilon / 2 & \text { if } & y<-1
\end{array}\right.
$$




\section{Injetividade Global}

Neste capítulo provamos o Teorema E. Como um passo prévio estudaremos as folheações $\mathcal{F}_{i}$ em $\mathbb{R}^{n}$ induzidas pelas submersões $f_{i}$ que são as funções coordenadas de difeomorfismos locais $X=\left(f_{1}, \ldots, f_{n}\right): \mathbb{R}^{n} \rightarrow \mathbb{R}^{n}$. Em particular provamos no Teorema 3.3 a não compacidade das folhas nas interseções das folheações $\mathcal{F}_{i}$.

\subsection{Difeomorfismos locais e folheações}

Seja $X=\left(f_{1}, \ldots, f_{n}\right): \mathbb{R}^{n} \rightarrow \mathbb{R}^{n}$ uma aplicação de classe $C^{2}$ e $\operatorname{seja} \operatorname{Spec}(X)$ o conjunto de autovalores (complexos) da derivada $D X_{p}$ quando $p$ varia em todo $\mathbb{R}^{n}$. Se para todo $p \in \mathbb{R}^{n}, D X_{p}$ é não singular, ou equivalentemente $0 \notin \operatorname{Spec}(X)$ então cada função coordenada $f_{i}: \mathbb{R}^{n} \rightarrow \mathbb{R}$ é uma submersão e portanto, pela Proposição 1.10 , para cada $i \in\{1, \ldots, n\}$, as superfícies de nível $\left\{f_{i}=c t e\right\}$ compõem uma $C^{2}$-folheação $\mathcal{F}_{i}$ de codimensão 1 em $\mathbb{R}^{n}$.

Dada uma $k$-combinação $\left\{i_{1}<\ldots<i_{k}\right\}$ do conjunto $\{1, \ldots, n\}$, desde que a aplicação $X_{i_{1}, \ldots, i_{k}}=\left(f_{i_{1}}, \ldots, f_{i_{k}}\right): \mathbb{R}^{n} \rightarrow \mathbb{R}^{k}$ é uma $C^{2}$-submersão, novamente pela Proposição 1.10, as superfícies de nível $(n-k)$-dimensionais $X_{i_{1}, \ldots, i_{k}}=\left(c_{1}, \ldots, c_{k}\right)$ formam uma $C^{2}$-folheação $\mathcal{F}_{i_{1} \ldots i_{k}}$ do $\mathbb{R}^{n}$ de codimensão $k$. Por outro lado, como as folheações $\mathcal{F}_{i_{1}}, \ldots, \mathcal{F}_{i_{k}}$ são transversais entre si, a interseção $\mathcal{F}_{i_{1}} \cap \ldots \cap \mathcal{F}_{i_{k}}$ gera uma partição do $\mathbb{R}^{n}$ em subvariedades imersas. Além disto, está partição coincide com a dada pela folheação $\mathcal{F}_{i_{1} \ldots i_{k}}$.

A seguir enunciamos o Teorema de Jordan-Brouwer, que é uma generalização do Teorema da curva de Jordan. Uma demonstração para o caso de superfícies contínuas baseada na sequência de Mayer-Vietoris pode ser encontrada em [59]:

Teorema 3.1. [Teorema de Jordan-Brouwer] Se $M \subset \mathbb{R}^{n}$ é uma superfície de dimensão $n-1$ conexa e compacta, então $\mathbb{R}^{n} \backslash M=A \cup B$ é a reunião de dois abertos conexos disjuntos que têm $M$ como fronteira comum, sendo um dos abertos limitado e o outro 
ilimitado.

Quando $n=2$, este teorema reduz-se ao Teorema da Curva de Jordan. Note que é indiferente considerar $M$ contida no espaço euclidiano $\mathbb{R}^{n}$ ou na esfera $S^{n} \cup\{\infty\}$. Estamos interessados na seguinte consequência deste resultado:

Proposição 3.2. Seja $X=\left(f_{1}, \ldots, f_{n}\right): \mathbb{R}^{n} \rightarrow \mathbb{R}^{n}$ uma aplicação de classe $C^{2}$ tal que $\operatorname{Spec}(X) \cap\{0\}=\varnothing$. Então as folhas das folheações $\mathcal{F}_{i}$ são não compactas para todo $i \in\{1, \ldots, n\}$.

Dem.: Se existisse uma folha compacta em $\mathcal{F}_{i}$, para algum $i \in\{1, \ldots, n\}$, então, pelo Teorema de Jordan-Brouwer 3.1, seu complemento contém uma componente limitada $N$. Assim, $\inf \left\{f_{i}(p) ; p \in \bar{N}\right\}$ ou $\sup \left\{f_{i}(p) ; p \in \bar{N}\right\}$ é um ponto crítico para $f_{i}: \mathbb{R}^{n} \rightarrow \mathbb{R}$ o que contradiz o fato desta função ser uma submersão.

No próximo teorema provamos que tal propriedade é satisfeita também pelas folheações intersectadas $\mathcal{F}_{i_{1} \ldots i_{k}}$.

Teorema 3.3. Seja $X=\left(f_{1}, \ldots, f_{n}\right): \mathbb{R}^{n} \rightarrow \mathbb{R}^{n}$ uma aplicação de classe $C^{2}$ tal que $\operatorname{Spec}(X) \cap\{0\}=\varnothing$. Então as folheações $\mathcal{F}_{i_{1} \ldots i_{k}}$ não possuem folhas compactas, onde $k \in\{1, \ldots, n-1\}$ e $\left\{i_{1}, \ldots, i_{k}\right\}$ é uma $k$-combinação arbitrária de $\{1, \ldots, n\}$.

Dem.: Aplicaremos o princípio de indução finita na dimensão $n-k$ das folhas. Para dimensão 1 temos $k=n-1$. Suponha que $\ell$ é uma folha compacta de $\mathcal{F}_{i_{1} \ldots i_{n-1}}$. Desde que $\ell$ é uma variedade compacta unidimensional, $\ell$ é homeomorfa à esfera unidimensional $S^{1}$. Observe que $\ell$ está contido em alguma folha dois dimensional $L$ de $\mathcal{F}_{i_{1} \ldots i_{n-2}}$. De fato, sendo $X$ não singular temos que $\ell$ é uma folha da folheação regular $\left.\mathcal{F}_{i_{n-1}}\right|_{L}$, a qual é induzida pela submersão $\left.f_{i_{n-1}}\right|_{L}: L \rightarrow \mathbb{R}$ e portanto tem holonomia trivial. Assim, pelo Teorema 1.15 (de estabilidade local de Reeb), existe uma vizinhança $U$ de $\ell$ em $L$ tal que toda folha de $\left.\mathcal{F}_{i_{n-1}}\right|_{L}$ passando por algum ponto de $U$ é homeomorfa a $S^{1}$.

Desde que $\left.\mathcal{F}_{i_{n}}\right|_{L}$ é transversal a $\left.\mathcal{F}_{i_{n-1}}\right|_{L}$, as folhas de $\left.\mathcal{F}_{i_{n}}\right|_{L}$ restritas a $U$ são curvas começando em uma das componentes conexas de $\partial U$ e terminando na outra. Além disto, as folhas de $\left.\mathcal{F}_{i_{n-1}}\right|_{L}$ passando por $U$ são homotopicamente não nulas em $L$.

Seja $\eta: D^{2} \rightarrow \mathbb{R}^{n}$ uma imersão de classe $C^{2}$ do disco bidimensional $D^{2}=\{(x, y) \in$ $\left.\mathbb{R}^{2} ; x^{2}+y^{2} \leq 1\right\}$ em $\mathbb{R}^{n}$ cuja imagem contém $\ell$. Pelo Teorema 1.21 podemos assumir que $\eta$ está em posição geral com respeito a $\mathcal{F}_{i_{n}}$. Seja $\mathcal{G}$ a folheação (com singularidades) em $D^{2}$ induzida por $\mathcal{F}_{i_{n}}$ via $\eta$. Afirmamos que é possível construir um campo vetorial $G$ em 
$D^{2}$ tal que a folheação $\mathcal{G}$ é induzida pelas órbitas de $G$. De fato, como $\eta$ está em posição geral com respeito a $\mathcal{F}_{i_{n}}$, a folheação $\mathcal{G}$ tem finitas singularidades sendo cada uma delas localmente topologicamente equivalentes a um centro ou a uma sela de um campo vetorial. Isto implica que $\mathcal{G}$ é localmente orientável em todo ponto. Pela Proposição 1.22, $\mathcal{G}$ é globalmente orientável. Isto prova a existência do campo vetorial $G$. Aplicando novamente o Teorema 1.21, podemos escolher $\eta$ de tal forma que nenhum par de singularidades de $G$ pertença à mesma folha de $\mathcal{F}_{i_{n}}$, em particular $G$ não possui conexões entre selas.

Observe que por um lado temos que $G$ é transversal a $\ell$. E por outro, $G$ não possui ciclos limites, senão existiria uma folha de $\mathcal{G}$ espiralando em torno de um ciclo limite $\gamma$ e a folha de $\mathcal{F}_{i_{n}}$ contendo $\gamma$ teria grupo de holonomia não trivial, o que é uma contradição pela Observação 1.13. Portanto, as singularidades de $G$ não podem ser centros nem selas, o que contradiz o fato de $D^{2}$ estar em posição geral com respeito a $\mathcal{F}_{i_{n}}$. Tal contradição prova que $\ell$ não é compacta e portanto o primeiro passo de indução.

Por hipótese de indução, suponha que as folhas das folheações de dimensão $r=n-k$ com $1<r<n-1$ são não compactas. Vamos provar que as folheações $(r+1)$-dimensionais também não possuem folhas compactas.

Suponha, por absurdo, que existe uma folha $L(r+1)$-dimensional compacta de $\mathcal{F}_{i_{1} \ldots i_{k-1}}$, para alguma $k$-combinação $\left\{i_{1}, \ldots, i_{k}\right\}$ de $\{1, \ldots, n\}$. Desde que $X$ é não singular, $\mathcal{F}_{i_{k}} \mid L$ é uma folheação sem holonomia cujas folhas são também folhas da folheação $\mathcal{F}_{i_{1} \ldots i_{k}}$, em particular são de dimensão $r$. Logo, $\mathcal{F}_{i_{k}} \mid L$ não possui folhas compactas.

Pelo Teorema 1.172 ), a folheação $\mathcal{F}_{i_{k}} \mid L$ possui um conjunto minimal. Desde que as folhas de tal folheação são não compactas, um conjunto minimal $M$ pode ser todo $L$ ou um subconjunto próprio de $L$ que não seja uma única folha. Porém, aplicando o item 3) do Teorema 1.17 no primeiro caso e o Teorema 1.18 no segundo, concluímos que a holonomia de $\mathcal{F}_{i_{k}} \mid L$ é não trivial, o que contradiz com o fato de folheações induzidas por submersões têm holonomia trivial. Esta contradição prova que $L$ não pode ser compacta.

\subsection{Versão fraca da Conjetura Jacobiana real de Jelonek}

Para estabelecer nosso resultado de injetividade global, vamos introduzir alguns conceitos e resultados prévios.

Definição 3.4. Seja $X: M \rightarrow N$ uma aplicação contínua entre espaços localmente compactos. Dizemos que a aplicação $X$ é não própria no ponto $p \in N$, se para qualquer vizinhança $U$ de $p$ a pré-imagem $X^{-1}(\bar{U})$ é não compacta. 
O conjunto $S_{X}$ dos pontos de $N$ nos quais a aplicação $X$ é não própria indica o quanto a aplicação $X$ difere de uma aplicação própria. Em particular, $X$ é própria se e somente se este conjunto for vazio. Além disto, se $X(M)$ é aberta, então $S_{X}$ contém o bordo de $X(M)$. O conjunto $S_{X}$ é o menor conjunto $S$ com a propriedade de que a aplicação $X: M \backslash X^{-1}(S) \rightarrow N \backslash S$ é própria.

O seguinte resultado devido a Hadamard revela que é estreita a relação entre o conjunto não próprio de uma aplicação e o fato de tal aplicação ser globalmente injetiva.

Teorema 3.5. Um difeomorfismo local $X: \mathbb{R}^{n} \rightarrow \mathbb{R}^{n}$ de classe $C^{1}$ é um difeomorfismo se, e somente se, é uma aplicação própria (ou seja, $S_{X}$ é vazio).

No âmbito das aplicações polinomiais, Jelonek provou em [36]:

Teorema 3.6. Se $X: \mathbb{R}^{n} \rightarrow \mathbb{R}^{n}$ é uma aplicação polinomial satisfazendo $\operatorname{Spec}(X) \cap\{0\}=$ $\varnothing e \operatorname{codim}\left(S_{X}\right) \geq 3$, então $X$ é bijetiva (e consequentemente $S_{X}=\varnothing$ ).

Recordamos o seguinte resultado devido a Bialynicki-Birula e Rosenlicht [8]:

Teorema 3.7. Se $X: \mathbb{R}^{n} \rightarrow \mathbb{R}^{n}$ é uma aplicação polinomial injetiva, então $X$ é bijetiva.

Portanto, para obter a bijetividade é suficiente provar a injetividade de $X$. Por outro lado, se $P: \mathbb{R}^{2} \rightarrow \mathbb{R}^{2}$ é um difeomorfismo local polinomial do plano não injetivo, segue pelo Teorema 3.5 que $S_{P} \neq \varnothing$ e pelo Teorema $3.9 \operatorname{temos}$ que $\operatorname{dim}\left(S_{P}\right)=\operatorname{codim}\left(S_{P}\right)=1$. O famoso exemplo de Pinchuck dado em [49] satisfaz tais condições.

Exemplo 3.8. Seja $P: \mathbb{R}^{2} \rightarrow \mathbb{R}^{2}$ um difeomorfismo local polinomial não injetivo. Então a aplicação

$$
X\left(x_{1}, \ldots, x_{n}\right)=\left(P\left(x_{1}, x_{2}\right), x_{3}, \ldots, x_{n}\right),
$$

é um difeomorfismo local polinomial $X: \mathbb{R}^{n} \rightarrow \mathbb{R}^{n}$ com $\operatorname{codim}\left(S_{X}\right)=1$ e portanto não injetivo, para todo $n \geq 3$.

Portanto, o único caso de interesse é quando $\operatorname{codim}\left(S_{X}\right)=2$ ou $\operatorname{codim}\left(S_{X}\right)=1$ com hipóteses adicionais. Estes fatos motivaram Jelonek a estabelecer em [36] a seguinte:

Conjetura Jacobiana real de Jelonek: Seja $X: \mathbb{R}^{n} \rightarrow \mathbb{R}^{n}$ uma aplicação polinomial tal que $\operatorname{Spec}(X) \cap\{0\}=\varnothing$. Se $\operatorname{codim}\left(S_{X}\right) \geq 2$ então $X$ é bijetiva.

Um subconjunto do espaço $\mathbb{R}^{n}$ é dito semi-algébrico se for da forma

$$
\bigcup_{i=1}^{s} \bigcap_{j=1}^{r_{i}}\left\{x \in \mathbb{R}^{n} ; f_{i, j} *_{i, j} 0\right\},
$$


onde $f_{i, j}: \mathbb{R}^{n} \rightarrow \mathbb{R}$ são funções polinomiais e $*_{i, j}$ é $<$ ou $=$, para todo $i=1, \ldots, s$ e $j=1, \ldots, r_{i}$. Ainda em [36], Jelonek provou o seguinte teorema, do qual se conclui que sua conjetura é verdadeira no caso bidimensional.

Teorema 3.9. Se $X: \mathbb{R}^{n} \rightarrow \mathbb{R}^{n}$ é uma aplicação polinomial não constante, então o conjunto $S_{X}$ é fechado, semi-algébrico e para toda componente conexa $S \subset S_{X}$ temos $1 \leq \operatorname{dim}(S) \leq n-1$. Além disto, para cada ponto $q \in S_{X}$ existe uma aplicação polinomial $\phi: \mathbb{R} \rightarrow S_{X}$ tal que $\phi(\mathbb{R})$ é uma curva semi-algébrica passando por $q$.

Além disto, Gutierrez e Maquera provaram em [28] uma versão fraca da Conjetura Jacobiana Real de Jelonek no caso tridimensional, considerando adicionalmente a hipótese espectral: $\operatorname{Spec}(X) \cap[0, \epsilon)=\varnothing$, para algum $\epsilon>0$.

Outro fato, provado por Jelonek em [36], que ilustra a relevância de sua conjetura é que uma resposta afirmativa dela implica na solução da famosa:

Conjetura Jacobiana: Seja $X: \mathbb{C}^{n} \rightarrow \mathbb{C}^{n}$ uma aplicação polinomial com Jacobiano não nulo em todo ponto, então $X$ é um isomorfismo, isto é, invertível com inversa polinomial.

A fim de estabelecer os principais teoremas deste capítulo serão necessários ainda os seguintes resultados:

Lema 3.10. Seja $X: \mathbb{R}^{n} \rightarrow \mathbb{R}^{n}$ uma aplicação de classe $C^{1}$ tal que $\operatorname{Spec}(X) \cap\{0\}=$ $\varnothing$ e o grupo fundamental $\pi_{1}(\ell)$ é trivial para toda folha $\ell \in \mathcal{F}_{i_{1} \ldots i_{k}}$, onde $\left\{i_{1}, \ldots, i_{k}\right\}$ é uma $k$-combinação de $\{1, \ldots, n\}$. Seja $A: \mathbb{R}^{n} \rightarrow \mathbb{R}^{n}$ um isomorfismo linear. Se $Z=A \circ X \circ A^{-1}$ então $\operatorname{Spec}(X)=\operatorname{Spec}(Z), S_{Z}=A\left(S_{X}\right)$ e $\pi_{1}\left(\ell_{Z}\right)$ é trivial, para toda $\ell_{Z} \in \mathcal{F}_{i_{1} \ldots i_{k}}^{Z}$, onde $\mathcal{F}^{Z}$ indica folheações induzidas pelas funções coordenadas de $Z$.

Dem.: Desde que $D Z=D A . D X .(D A)^{-1}$, temos que $D Z$ e $D X$ são matrizes similares e portanto possuem os mesmos autovalores. Portanto, $\operatorname{Spec}(X)=\operatorname{Spec}(Z)$.

Vamos mostrar que $A\left(S_{X}\right) \subset S_{Z}$. Dados $p \in S_{X}$ e $W$ uma vizinhança de $A(p)$, temos

$$
Z^{-1}(\bar{W})=\left(A \circ X \circ A^{-1}\right)^{-1}(\bar{W})=A \circ X^{-1}\left(A^{-1} \bar{W}\right) .
$$

Desde que $A^{-1} \bar{W}$ é uma vizinhança de $p \in S_{X}$ e $A$ é um homeomorfismo, temos que $X^{-1}\left(A^{-1} \bar{W}\right)$ e portanto $Z^{-1}(\bar{W})$ não são compactos, implicando $A(p) \in S_{Z}$.

Provemos agora que $S_{Z} \subset A\left(S_{X}\right)$. Seja $q \in S_{Z}$, observe que basta mostrar que $A^{-1}(q) \in S_{X}$. Dada $V$ vizinhança de $q$ temos que $A(V)$ é vizinhança de $q$ e portanto o conjunto

$$
W=Z^{-1}(\overline{A(V)})=A \circ X^{-1}(\bar{V})
$$


é não compacto. Sendo $A$ um homeomorfismo concluimos que $X^{-1}(\bar{V})=A^{-1}(W)$ é não compacto e $A^{-1} q \in S_{X}$.

A trivialidade de $\pi_{1}(\ell)$ segue do fato de ser $\pi_{1}$ um invariante topológico e as folheações $\mathcal{F}_{i_{1} \ldots i_{k}}$ e $\mathcal{F}_{i_{1} \ldots i_{k}}^{Z}$ serem topologicamente conjugadas.

O próximo resultado dado por Drużkowski e Tutaj em [17] fornece uma estimativa da cardinalidade da pré-imagem de um ponto qualquer para um difeomorfismo local polinomial.

Teorema 3.11. Seja $X=\left(f_{1}, \ldots, f_{n}\right): \mathbb{R}^{n} \rightarrow \mathbb{R}^{n}$ um difeomorfismo local polinomial. Então, para todo $q \in \mathbb{R}^{n}$, a equação $X(p)=q$ tem finitas soluções $e$

$$
\#\left\{p \in \mathbb{R}^{n} ; X(p)=q \leq \prod_{i=1}^{n} g r\left(f_{i}\right)\right\} .
$$

Finalmente, mostramos um fato topológico que relaciona os pontos onde um difeomorfismo local polinomial é próprio com pontos em que a função cardinalidade de pré-imagens é localmente constante.

Lema 3.12. Seja $X: \mathbb{R}^{n} \rightarrow \mathbb{R}^{n}$ um difeomorfismo local polinomial. Então $X$ é uma aplicação própria no ponto $p_{0} \in \mathbb{R}^{n}$ se, e somente se, a função $h: \mathbb{R}^{n} \rightarrow \mathbb{N}$ que a cada $p \in \mathbb{R}^{n}$ associa $h(p)=\# X^{-1}(p)$ é localmente constante em $p_{0}$.

Dem.: Seja $p_{0}$ um ponto próprio de $X$, então existe uma vizinhança $U$ de $p_{0}$ tal que $X^{-1}(\bar{U})$ é um conjunto compacto. Sendo $X$ difeomorfismo local polinomial temos, pelo Teorema 3.11, que $\# X^{-1}\left(p_{0}\right)$ é finita. Além disto, para uma vizinhança $V$ suficientemente pequena de $p_{0}$ temos que $\# X^{-1}(p) \geq \# X^{-1}\left(p_{0}\right)$, para todo $p \in V$.

Afirmamos que existe uma vizinhança $V$ de $p_{0}$ tal que $\# X^{-1}(p)=\# X^{-1}\left(p_{0}\right)$, para todo $p \in V$. De fato, se tal vizinhança não existisse, seria possível construir uma sequência $\left(p_{n}\right) \subset U$ convergindo para $p$ tal que sua pré-imagem $X^{-1}\left(p_{n}\right)$ possui uma sequência $q_{n}$ que não converge para nenhuma pré-imagem de $p$. Logo, $q_{n} \rightarrow \infty$ e $X^{-1}(\bar{U})$ não seria compacto.

Suponhamos agora que $\# X^{-1}\left(p_{0}\right)$ é localmente constante. Como $X$ é um difeomorfismo local polinomial, então existe uma vizinhança $W$ de $p_{0}$ tal que $k=\# X^{-1}(p)=$ $\# X^{-1}\left(p_{0}\right)$ para todo $p \in U$. Podemos escolher $W$ de tal forma que $X^{-1}(W)=\cup_{i=1}^{k} W_{i}$, onde $W_{i}$ são abertos disjuntos de $\mathbb{R}^{n}$. Além disto, é possível escolher abertos $V_{i} \subset W_{i}$, de tal forma que $V_{i} \cap X^{-1}(W) \neq \varnothing$ e $\left.X\right|_{V_{i}}: V_{i} \rightarrow X\left(V_{i}\right) \subset W$ é um difeomorfismo, para cada $i=1, \ldots, k$. Assim, a vizinhança $U$ dada por $\cap_{i=1}^{k} X\left(V_{i}\right)$ contém o ponto $p_{0}$ e a 
pré-imagem $X^{-1}(\bar{U})$ é compacta, isto é, $X$ é própria em $p_{0}$.

A seguir provamos o principal resultado do presente capítulo. Salientamos que daremos primeiro uma demonstração no caso de dimensão 4. É claro que este resultado "está contido" no Teorema E, porém ele foi mantido aqui por ser sua prova intuitivamente mais instrutiva que a do caso $n$-dimensional.

Teorema 3.13. Seja $X=\left(f_{1}, \ldots, f_{4}\right): \mathbb{R}^{4} \rightarrow \mathbb{R}^{4}$ uma aplicação polinomial tal que $\operatorname{Spec}(X) \cap\{0\}=\varnothing$ e $\pi_{1}(\ell)$ é trivial, para toda folha $\ell \in \mathcal{F}_{i j}$ e todo $i, j \in\{1, \ldots, 4\}$ com $i \neq j$. Se $\operatorname{codim}\left(S_{X}\right) \geq 2$ então X é uma bijeção.

Dem.: Suponha que $X$ não é uma aplicação bijetiva. Pelo Teorema 3.6, temos que $\operatorname{codim}\left(S_{X}\right)=\operatorname{dim}\left(S_{X}\right)=2$. Sendo $X$ um difeomorfismo local, $X\left(\mathbb{R}^{4}\right)$ é aberto e como observado após à Definição 3.4 segue que $S_{X}$ contém o bordo de $X\left(\mathbb{R}^{4}\right)$, isto implica que

(a) $X\left(\mathbb{R}^{4}\right) \supset \mathbb{R}^{4} \backslash S_{X}$.

De fato, se existe $p \in \mathbb{R}^{4} \backslash\left\{S_{X} \cup X\left(\mathbb{R}^{4}\right)\right\}$, como $S_{X} \supset \partial X\left(\mathbb{R}^{4}\right)$, segue que $p \notin \partial X\left(\mathbb{R}^{4}\right)$. Logo, existe uma vizinhança $V$ de $p$ em $\mathbb{R}^{4}$ tal que $X\left(\mathbb{R}^{4}\right) \cap V=\varnothing$. Desde que $X\left(\mathbb{R}^{4}\right)$ é aberto de $\mathbb{R}^{4}$ e $S_{X} \supset \partial X\left(\mathbb{R}^{4}\right)$ temos que o bordo $\partial X\left(\mathbb{R}^{4}\right)$ e, em particular, alguma componente conexa de $S_{X}$ tem dimensão 3 . Esta contradição mostra a afirmação (a).

Analogamente a Gutierrez e Maquera em [28], construiremos uma vizinhança compacta $W$ em $\mathbb{R}^{4}$ de um ponto não próprio $p \in S_{X}$ tal que sua pré-imagem é compacta, tal contradição prova o teorema.

Pelo Teorema 3.9 temos que $S_{X}$ contém uma curva polinomial $\gamma:\left(a_{1}-\delta_{1}, a_{1}+\delta_{1}\right) \rightarrow$ $S_{X} \subset \mathbb{R}^{4}$. Pelo Lema 3.10 , podemos supor que tal curva intersecta transversalmente o hiperplano $\left\{x_{1}=a_{1}\right\}$ no ponto $p=\gamma\left(a_{1}\right)=\left(a_{1}, \ldots, a_{4}\right)$ (veja Figura 3.1).

Além disto, para um $\delta_{2}>0$ suficientemente pequeno, temos que $D\left(a_{1}\right)=\left\{a_{1}\right\} \times D \subset$ $\left\{x_{1}=a_{1}\right\}$, onde $D$ é o disco tridimensional de $\mathbb{R}^{3}$ centrado em $\left(a_{2}, a_{3}, a_{4}\right)$ de raio $\delta_{2}$, satisfaz:

(b) $\gamma\left(a_{1}\right)=D\left(a_{1}\right) \cap \gamma, C\left(a_{1}\right) \cap \gamma=\varnothing$, onde $C\left(a_{1}\right)$ é o bordo de $D\left(a_{1}\right)$, e a projeção de $D\left(a_{1}\right) \cap S_{X}$ sobre o eixo $x_{2}$ é injetiva.

Em particular, existem $\epsilon_{1}, \epsilon_{2}>0$ tais que a seguinte restrição da projeção na segunda coordenada

$$
\left.\Pi_{2}\right|_{D\left(a_{1}\right) \cap S_{X}}: D\left(a_{1}\right) \cap S_{X} \rightarrow\left[a_{2}-\epsilon_{1}, a_{2}+\epsilon_{2}\right]
$$




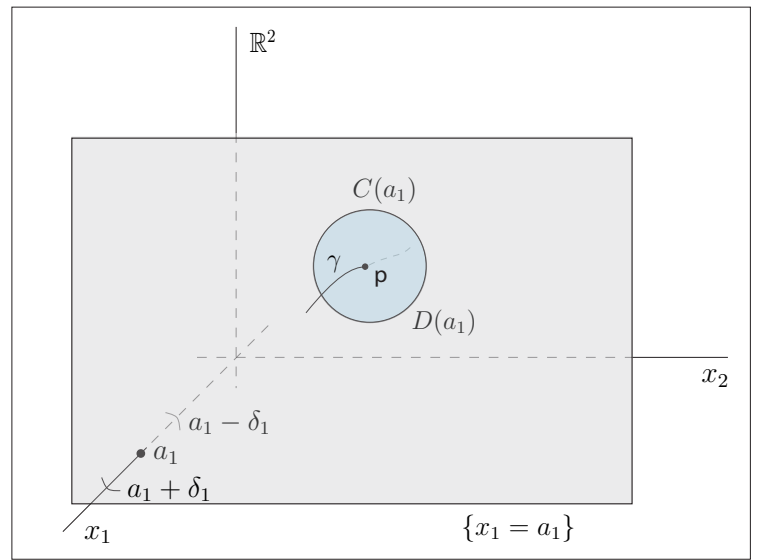

Figura 3.1

é uma aplicação bijetiva e portanto um homeomorfismo cuja inversa será denotada por:

$$
\varphi:\left[a_{2}-\epsilon_{1}, a_{2}+\epsilon_{2}\right] \rightarrow D\left(a_{1}\right) \cap S_{X},
$$

a qual associa cada $t \in\left[a_{2}-\epsilon_{1}, a_{2}+\epsilon_{2}\right]$ a $\varphi(t)=\left(\left.\Pi_{2}\right|_{D\left(a_{1}\right) \cap S_{X}}\right)^{-1}(t)=\Pi_{2}^{-1}(t) \cap D\left(a_{1}\right) \cap S_{X}$ (cf. Figura 3.2p).

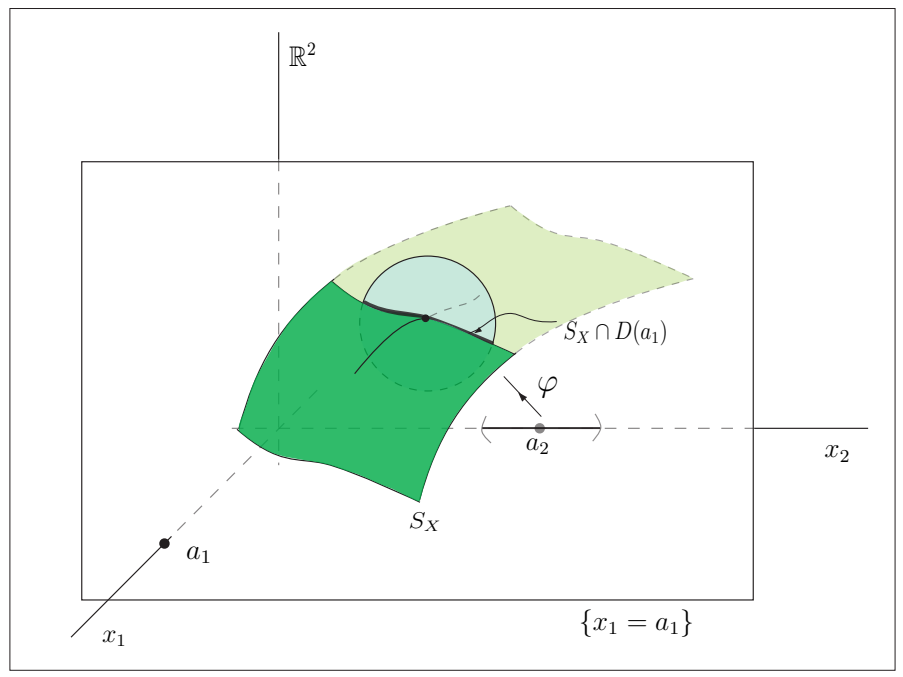

Figura 3.2 
Assim, a cada $t \in\left(a_{2}-\epsilon_{1}, a_{2}+\epsilon_{2}\right)$ podemos associar um único disco bidimensional $B\left(a_{1}, t\right) \subset D\left(a_{1}\right) \cap\left\{x_{2}=t\right\}$ satisfazendo $B\left(a_{1}, t\right) \cap S_{X}=\{\varphi(t)\}$ e $\partial B\left(a_{1}, t\right) \cap S_{X}=\varnothing$, onde $\partial B\left(a_{1}, t\right)$ é o bordo de $B\left(a_{1}, t\right)$ (ver Figura 3.3).

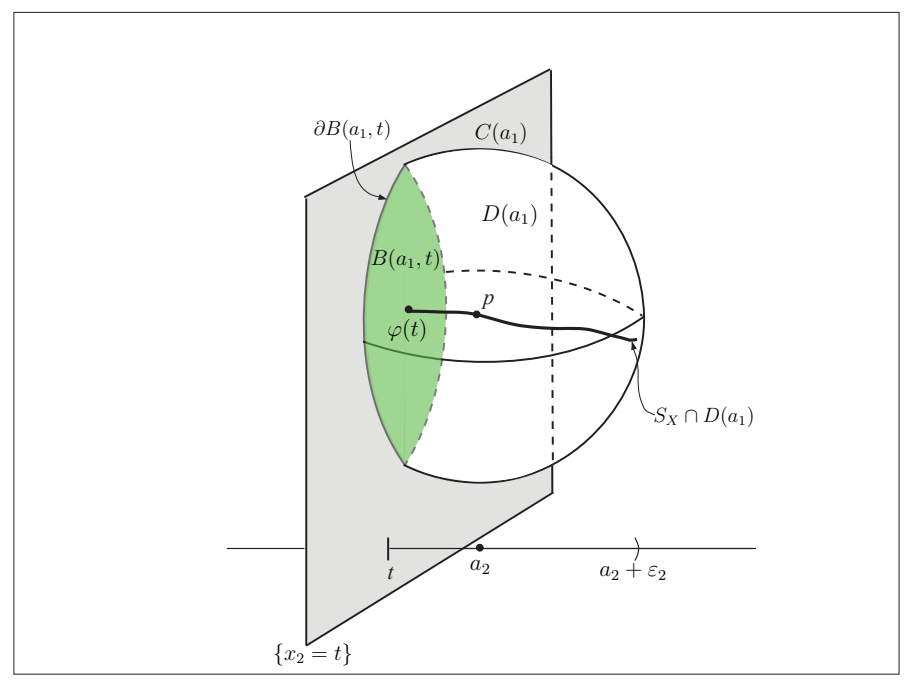

Figura 3.3

(c) Pelo Lema 3.12, \# $X^{-1}$ é localmente constante em pontos próprios de $X$. Além disto, do Teorema 3.11, existe um inteiro positivo $K$ tal que para todo $q \in \mathbb{R}^{4}$, $\# X^{-1}(q) \leq K$.

Assim, temos que o difeomorfismo local $X$ restrito a $X^{-1}\left(\partial B\left(a_{1}, t\right)\right)$ é um recobrimento finito de $\partial B\left(a_{1}, t\right)$ e portanto $X^{-1}\left(\partial B\left(a_{1}, t\right)\right)$ é a união finita de círculos mergulhados $C_{1}\left(a_{1}, t\right), \ldots, C_{k}\left(a_{1}, t\right)$ contidos em $f_{1}^{-1}\left(a_{1}\right) \cap f_{2}^{-1}(t)$, isto é, contidos em folhas de $\mathcal{F}_{12}$. É claro que $k \leq K$. Como, por hipótese, cada componente conexa de $f_{1}^{-1}\left(a_{1}\right) \cap f_{2}^{-1}(t)$ é uma variedade simplesmente conexa bidimensional de $\mathbb{R}^{4}$ e pelo Teorema 3.3 é não compacta, temos que tais folhas são planos. Portanto, para cada $i=1,2, \ldots, k$ existe um disco bidimensional $D_{i}\left(a_{1}, t\right) \subset f_{1}^{-1}\left(a_{1}\right) \cap f_{2}^{-1}(t)$ bordado por $C_{i}\left(a_{1}, t\right)$. Disto segue que $X\left(D_{i}\left(a_{1}, t\right)\right)=B\left(a_{1}, t\right)$, para todo $i \in\{1, \ldots, k\}$. Desde que $B(t)$ é simplesmente conexo $\left.X\right|_{D_{i}\left(a_{1}, t\right)}: D_{i}\left(a_{1}, t\right) \rightarrow B\left(a_{1}, t\right)$ é uma aplicação bijetiva e portanto um difeomorfismo para todo $i \in\{1, \ldots, k\}$.

Como $B\left(a_{1}, t\right) \cap S_{X}=\varphi(t)$ e \# $X^{-1}$ é localmente constante em pontos próprios, \# $X^{-1}$ é identicamente igual a $k$ em $B\left(a_{1}, t\right) \backslash\{\varphi(t)\}$. Logo, $X^{-1}\left(B\left(a_{1}, t\right) \backslash\{\varphi(t)\}\right) \subset \cup_{i=1}^{k} D_{i}\left(a_{1}, t\right)$. Por outro lado, $\# X^{-1}(\varphi(t)) \geq k$, e desde que $X$ é um difeomorfismo local, concluímos que 
(d) para todo $q \in B\left(a_{1}, t\right), \# X^{-1}(q)=k$ e $X^{-1}\left(B\left(a_{1}, t\right)\right)=\cup_{i=1}^{k} D_{i}\left(a_{1}, t\right)$.

Tomando $\bar{\delta}_{2}>0$ tal que $\bar{\delta}_{2}<\epsilon_{j}, j=1,2$, acabamos de provar que para todo $t \in\left[a_{2}-\bar{\delta}_{2}, a_{2}+\bar{\delta}_{2}\right]=I_{2}, X^{-1}\left(B\left(a_{1}, t\right)\right)=\cup_{i=1}^{k} D_{i}\left(a_{1}, t\right)$ (para o mesmo $k$ ), onde $B\left(a_{1}, t\right) \mathrm{e}$ $D_{i}\left(a_{1}, t\right)$ são como previamente definidos. Assim, podemos definir $W\left(a_{1}\right)=\cup_{t \in I_{2}}\left(B\left(a_{1}, t\right)\right)$ que é um conjunto compacto tridimensional contendo $\gamma\left(a_{1}\right)$, contido em $D\left(a_{1}\right)$ e tal que $X^{-1}\left(W\left(a_{1}\right)\right)$ é uma união de de $k$ conjuntos compactos tridimensionais em $\mathbb{R}^{4}$.

Note que reduzindo $\delta_{1}$, se necessário, temos que

(e) para todo $s \in\left[a_{1}-\delta_{1}, a_{1}+\delta_{1}\right]=I_{1}$, o disco tridimensional $D(s)=\{s\} \times D$ e seu bordo $C(s)=\{s\} \times \partial D$, onde $D$ é como previamente definido, satisfaz $\{\gamma(s)\}=D(s) \cap \gamma$ e $C(s) \cap \gamma=\varnothing$.

Procedendo como acima, podemos construir um conjunto compacto tridimensional $W(s)$, para cada $s \in I_{1}$, contido em $D(s)$ tal que $X^{-1}(W(s))$ é a união de $k$ conjuntos compactos tridimensionais em $\mathbb{R}^{4}$. De fato, dado $s \in I_{1}$ podemos tomar de maneira contínua um $\bar{\delta}_{2}(s)$ tal que $W(s)=\cup_{t \in I_{2}(s)} B(s, t)$, onde $B(s, t)$ é o análogo ao disco $B\left(a_{1}, t\right)$, $I_{2}(s)=\left[a_{2}-\bar{\delta}_{2}(s), a_{2}+\bar{\delta}_{2}(s)\right]$, contém $\gamma(s)$ e satisfaz todas propriedades requeridas.

Desde que $\bar{\delta}_{2}: I_{1} \rightarrow \mathbb{R}_{+}$é uma função contínua definida num compacto que nunca atinge o 0 , temos que $\delta=\inf _{s \in I_{1}}\left\{\bar{\delta}_{2}(s)\right\}$ é positivo. Desta forma, para todo $s \in I_{1}$ o conjunto $W(s)=\cup_{t \in\left[a_{2}-\delta, a_{2}+\delta\right]} B(s, t)$ tem como pré-imagem de $X$ uma união de $k$ conjuntos compactos tridimensionais em $\mathbb{R}^{4}$.

Portanto, desde que $W=\cup_{s \in I_{1}} W(s)$ é uma vizinhança compacta de $p=\gamma\left(a_{1}\right)$ em $\mathbb{R}^{4}$ tal que $X^{-1}(W)$ é compacto, obtemos uma contradição com o fato de $p \in S_{X}$.

Os argumentos utilizados na demonstração do próximo resultado serão os mesmos do Teorema 3.13, para $n=4$. Repassaremos resumidamente tais argumentos no intuito de ressaltar as diferenças que surgem para o caso $n>4$, tais diferenças basicamente estão associadas ao número de vezes que aplicaremos os passos descritos no caso $n=4$.

Teorema E. Seja $X=\left(f_{1}, \ldots, f_{n}\right): \mathbb{R}^{n} \rightarrow \mathbb{R}^{n}$ uma aplicação polinomial tal que $\operatorname{Spec}(X) \cap$ $\{0\}=\varnothing$ e para cada $(n-2)$-combinação $\left\{i_{1}, \ldots, i_{n-2}\right\}$ de $\{1, \ldots, n\}$, toda folha de $\mathcal{F}_{i_{1} \ldots i_{n-2}}$ é simplesmente conexa. Se $\operatorname{codim}\left(S_{X}\right) \geq 2$ então $X$ é uma bijeção.

Dem.: Para $n=1$ o resultado é trivial. Jelonek provou em [36] o caso $n=2$ usando somente a hipótese na $\operatorname{codim}\left(S_{X}\right)$. O caso $n=3$ é uma consequência do resultado de Gutierrez e Maquera em [28]. 
Supondo, por absurdo, que $X$ não é bijetiva, pelo Teorema 3.6 , temos que $\operatorname{codim}\left(S_{X}\right)=$ 2 , ou seja, $\operatorname{dim}\left(S_{X}\right)=n-2$. Como $X$ é um difeomorfismo local, $X\left(\mathbb{R}^{n}\right)$ é aberto e aplicando o Teorema 3.9 obtemos que

(a) $X\left(\mathbb{R}^{n}\right) \supset \mathbb{R}^{n} \backslash S_{X}$.

Pelo Teorema 3.9 e Lema 3.10, podemos supor que $S_{X}$ contém uma curva polinomial $\gamma:\left(a_{1}-\delta_{1}, a_{1}+\delta_{1}\right) \rightarrow \mathbb{R}^{n}$ intersectando transversalmente o hiperplano $\left\{x_{1}=a_{1}\right\}$ no ponto $p=\gamma\left(a_{1}\right)=\left(a_{1}, \ldots, a_{n}\right)$. Além disto, para um $\delta>0$ suficientemente pequeno, podemos assumir que o $(n-1)$-disco $D\left(a_{1}\right)=\left\{a_{1}\right\} \times D \subset\left\{x_{1}=a_{1}\right\}$, onde $D$ é o $(n-1)$-disco do $\mathbb{R}^{n-1}$ centrado em $\left(a_{2}, \ldots, a_{n}\right)$ com raio $\delta$, satisfaz:

(b) $\gamma\left(a_{1}\right)=D\left(a_{1}\right) \cap \gamma, C\left(a_{1}\right) \cap \gamma=\varnothing$, onde $C\left(a_{1}\right)$ é o bordo de $D\left(a_{1}\right)$, e $\Gamma=D\left(a_{1}\right) \cap$ $S_{X} \cap\left\{x_{2}=t_{2}\right\} \cap \ldots \cap\left\{x_{n-3}=t_{n-3}\right\}$, onde $t_{j} \in\left(a_{j}-\delta, a_{j}+\delta\right)$ está fixado para cada $j \in\{2, \ldots, n-3\}$, tem projeção no eixo $x_{n-2}$ injetiva. Ou seja, existem $\epsilon_{1}, \epsilon_{2}>0$ tais que

$$
\left.\Pi_{n-2}\right|_{\Gamma}: \Gamma \rightarrow\left[a_{n-2}-\epsilon_{1}, a_{n-2}+\epsilon_{2}\right]
$$

é bijetiva e portanto um homeomorfismo cuja inversa denotaremos por

$$
\varphi_{n-2}:\left[a_{n-2}-\epsilon_{1}, a_{n-2}+\epsilon_{2}\right] \rightarrow \Gamma
$$

a qual associa cada $t_{n-2} \in\left[a_{n-2}-\epsilon_{1}, a_{n-2}+\epsilon_{2}\right]$ a $\varphi_{n-2}\left(t_{n-2}\right)=\left(\left.\Pi_{n-2}\right|_{\Gamma}\right)^{-1}\left(t_{n-2}\right)=$ $\Pi_{n-2}^{-1}\left(t_{n-2}\right) \cap \Gamma$.

Assim, para cada $t_{n-2} \in\left(a_{n-2}-\epsilon_{1}, a_{n-2}+\epsilon_{2}\right)$ podemos associar um único 2-disco $B\left(a_{1}, t_{2}, \ldots, t_{n-2}\right) \subset D\left(a_{1}\right) \cap\left\{x_{2}=t_{2}\right\} \cap \ldots \cap\left\{x_{n-3}=t_{n-3}\right\} \cap\left\{x_{n-2}=t_{n-2}\right\}$ o qual satisfaz $B\left(a_{1}, t_{2}, \ldots, t_{n-2}\right) \cap S_{X}=\left\{\varphi_{n-2}\left(t_{n-2}\right)\right\}$ e $\partial B\left(a_{1}, t_{2}, \ldots, t_{n-2}\right) \cap S_{X}=\varnothing$, onde $\partial B\left(a_{1}, t_{2}, \ldots, t_{n-2}\right)$ é o bordo de $B\left(a_{1}, t_{2}, \ldots, t_{n-2}\right)$.

Desde que $\partial B\left(a_{1}, t_{2}, \ldots, t_{n-2}\right)$ é um círculo contido em $\left\{x_{1}=a_{1}\right\} \cap\left\{x_{2}=t_{2}\right\} \cap \ldots \cap$ $\left\{x_{n-2}=t_{n-2}\right\}$, temos que $X^{-1}\left(\partial B\left(a_{1}, t_{2}, \ldots, t_{n-2}\right)\right) \subset f_{1}^{-1}\left(a_{1}\right) \cap f_{2}^{-1}\left(t_{2}\right) \cap \ldots \cap f_{n-2}^{-1}\left(t_{n-2}\right)$. Isto significa que $X^{-1}\left(\partial B\left(a_{1}, t_{2}, \ldots, t_{n-2}\right)\right)$ está contida em folhas de $\mathcal{F}_{1,2, \ldots, n-2}$. Tais folhas são subvariedades de $\mathbb{R}^{n}$ 2-dimensionas que, por hipótese, simplesmente conexas e, pelo Teorema 3.3, são não compactas e portanto homeomorfas ao plano. Assim, seguindo o raciocínio em [28], ou equivalentemente, do Teorema 3.13, temos que

(c) $X^{-1}\left(\partial B\left(a_{1}, t_{2}, \ldots, t_{n-2}\right)\right)$ é a união finita de círculos mergulhados $C_{1}\left(a_{1}, t_{2}, \ldots, t_{n-2}\right), \ldots$, $C_{k}\left(a_{1}, t_{2}, \ldots, t_{n-2}\right)$ e cada um deles borda um disco bidimensional $D_{1}\left(a_{1}, t_{2}, \ldots, t_{n-2}\right)$, 
$\ldots, D_{k}\left(a_{1}, t_{2}, \ldots, t_{n-2}\right)$ contido em $f_{1}^{-1}\left(a_{1}\right) \cap f_{2}^{-1}\left(t_{2}\right) \cap \ldots \cap f_{n-2}^{-1}\left(t_{n-2}\right)$ satisfazendo:

$$
X^{-1}\left(B\left(a_{1}, t_{2}, \ldots, t_{n-2}\right)\right)=\cup_{i=1}^{k} D_{i}\left(a_{1}, t_{2}, \ldots, t_{n-2}\right) .
$$

Procedendo como no Teorema 3.13 podemos escolher $\delta_{n-2}>0$ tal que $\bar{\delta}_{n-2}<\epsilon_{j}, j=$ 1,2 , e para todo $t_{n-2} \in\left[a_{n-2}-\delta_{n-2}, a_{n-2}+\delta_{n-2}\right]=I_{n-2}$, temos que $X^{-1}\left(B\left(a_{1}, t_{2}, \ldots, t_{n-2}\right)\right)=$ $\cup_{i=1}^{k} D_{i}\left(a_{1}, t_{2}, \ldots, t_{n-2}\right)$ para o mesmo $k$, uma vez que o número de pré-imagens de $X$ é localmente constante em pontos próprios.

(d) Portanto o conjunto $W_{n-2}\left(a_{1}, t_{2}, \ldots, t_{n-3}\right)=\cup_{t_{n-2 \in I_{n-2}}} B\left(a_{1}, t_{2}, \ldots, t_{n-2}\right)$ é tridimensional, compacto, contém $p \in S_{X}$ e sua pré-imagem $X^{-1}\left(W_{n-2}\left(a_{1}, t_{2}, \ldots, t_{n-3}\right)\right)$ é uma união de $k$ conjuntos tridimensionais e compactos de $\mathbb{R}^{n}$.

Da mesma forma,

(e) podemos escolher $\delta_{n-3}>0$ tal que para todo $t_{n-3} \in\left[a_{n-3}-\delta_{n-3}, a_{n-3}+\delta_{n-3}\right]=I_{n-3}$, o conjunto $W_{n-2}\left(a_{1}, t_{2}, \ldots, t_{n-3}\right)$ como definido no item $(\mathrm{d})$ satisfaz todas as propriedades descritas em tal item. Logo, podemos definir o conjunto $W_{n-3}\left(a_{1}, t_{2}, \ldots, t_{n-4}\right)=$ $\cup_{t_{n-3} \in I_{n-3}} W_{n-2}\left(a_{1}, t_{2}, \ldots, t_{n-3}\right)$, que é um compacto de dimensão 4 contendo $p \in S_{X}$, tal que $X^{-1}\left(W_{n-3}\left(a_{1}, t_{2}, \ldots, t_{n-4}\right)\right)$ é uma união finita de conjuntos compactos de $\mathbb{R}^{n}$.

Após $n-3$ como em (e), construímos o conjunto $W_{2}\left(a_{1}\right)=\cup_{t_{2} \in I_{2}} W_{3}\left(a_{1}, t_{2}\right)$, que é um compacto de dimensão $(n-1)$ contendo $p \in S_{X}$ cuja pré-imagem $X^{-1}\left(W_{2}\left(a_{1}\right)\right)$ é a união de $k$ subconjuntos compactos de $\mathbb{R}^{n}$.

Finalmente, para $\delta_{1}>0$ pequeno o suficiente, podemos repetir o raciocínio de cada passo acima obtendo para cada $t_{1} \in\left[a_{1}-\delta_{1}, a_{1}+\delta_{1}\right]=I_{1}$ o conjunto $W_{2}\left(t_{1}\right)$, com as mesmas propriedades descritas no parágrafo anterior. Sendo assim, $W_{1}=\cup_{t_{1} \in I_{1}} W_{2}\left(t_{1}\right)$ é a vizinhança compacta de $p \in S_{X}$ em $\mathbb{R}^{n}$ que nos dá a contradição, uma vez que sua préimagem $X^{-1}\left(W_{1}\right)$ é uma união de $k$ conjuntos compactos e portanto compacta.

Para finalizar esta seção, ressaltamos que a hipótese topológica imposta sobre as folhas das folheações intersectadas de dimensão dois no teorema anterior, é análoga à condição obtida como consequência da hipótese espectral por Gutierrez e Maquera [28] no caso tridimensional:

Teorema 3.14. Seja $X=\left(f_{1}, f_{2}, f_{3}\right): \mathbb{R}^{3} \rightarrow \mathbb{R}^{3}$ uma aplicação de classe $C^{2}$. Se existe $\epsilon>0$ tal que $\operatorname{Spec}(X) \cap(-\epsilon, \epsilon)=\varnothing$ então cada folheação $\mathcal{F}_{i}$ é por planos, isto é, cada folha é $C^{2}$ difeomorfa a $\mathbb{R}^{2}$. 


\subsection{Versão fraca da Conjetura de Nollet e Xavier}

Dado um difeomorfismo local $X: \mathbb{R}^{n} \rightarrow \mathbb{R}^{n}$ estamos interessados em obter condições sob as quais $X$ é injetivo e portanto um difeomorfismo sobre sua imagem. Existe uma forte ligação entre os conceitos de injetividade e conexidade. Com efeito, da própria definição uma função é injetiva se, e somente se, a pré-imagem de todo ponto é conexa (possivelmente vazia). Por outro lado, não é difícil provar que um difeomorfismo local é injetivo se a pré-imagem de toda reta for conexa. Em vista destes fatos, Nollet e Xavier [45] conjeturaram:

Conjetura 3.15. Um difeomorfismo local $X: \mathbb{R}^{n} \rightarrow \mathbb{R}^{n}$ é injetivo se a pré-imagem de qualquer hiperplano afim é conexa (possivelmente vazia).

A relevância desta conjetura se expressa no fato de que se ela for verdadeira o mesmo ocorrerá com a Conjetura Jacobiana, como pode ser visto em [5]. Pelas observações feitas acima é fácil concluir que tal conjetura é verdadeira para $n \leq 2$, portanto o próximo caso de interesse é o tridimensional. Além disto, em [5] Balreira provou a seguinte versão fraca da conjetura de Nollet e Xavier:

Teorema 3.16 (Balreira). Um difeomorfismo local $X: \mathbb{R}^{n} \rightarrow \mathbb{R}^{n}$ é injetivo se a préimagem de qualquer hiperplano afim for vazia ou tiver a homologia de um ponto.

Como consequência deste teorema e do Teorema 3.14 provado por Gutierrez e Maquera em [28], obtemos a seguinte versão fraca da Conjetura de Nollet e Xavier em dimensão três:

Corolário 3.17. Um difeomorfismo local $X: \mathbb{R}^{3} \rightarrow \mathbb{R}^{3}$, de classe $C^{2}$, tal que $\operatorname{Spec}(X) \cap$ $(-\epsilon, \epsilon)=\varnothing$, para algum $\epsilon>0$, é injetivo se a pré-imagem de qualquer plano afim é vazia ou conexa.

Dem.: Pelo Teorema 3.16 basta mostrar que $X^{-1}(P)$ tem a homologia de um ponto, para todo plano afim $P \subset \mathbb{R}^{3}$.

Note que se um tal plano $P \subset \mathbb{R}^{3}$ for perpendicular a algum dos eixos coordenados $x_{i}$ de $\mathbb{R}^{3}$, ou seja, $P=\left\{\left(x_{1}, x_{2}, x_{3}\right) \in \mathbb{R}^{3} ; x_{i}=c\right\}$, teremos que se $X^{-1}(P)=f_{i}^{-1}(c)$ não for vazio então será conexo por hipótese e difeomorfo a $\mathbb{R}^{2}$ pelo Teorema 3.14 . Portanto, neste caso temos que a pré-imagem de $P$ tem a homologia de um ponto.

Por outro lado, dado um plano qualquer $P \subset \mathbb{R}^{3}$, é possível obter um isomorfismo linear $A: \mathbb{R}^{3} \rightarrow \mathbb{R}^{3}$ tal que $A(P)=P^{\prime}=\left\{\left(x_{1}, x_{2}, x_{3}\right) \in \mathbb{R}^{3} ; x_{i}=c\right\}$ para algum 
$i \in\{1,2,3\}$ e $c \in \mathbb{R}$. Além disto, note que $X^{-1}(P)$ tem homologia de um ponto se, e somente se, $A \circ X^{-1}(P)$ o tem. Por outro lado, desde que

$$
A \circ X^{-1}(P)=A \circ X^{-1} \circ A^{-1}\left(P^{\prime}\right)=\left(A \circ X \circ A^{-1}\right)^{-1}\left(P^{\prime}\right),
$$

$\operatorname{Spec}\left(A \circ X \circ A^{-1}\right)=\operatorname{Spec}(X)$ e $P^{\prime}$ é perpendicular ao eixo coordenado $x_{i}$ pelo visto acima temos que $A \circ X^{-1}(P)$, e portanto, $X^{-1}(P)$ é vazio ou tem a homologia de um ponto. 


\section{Considerações finais}

Neste capítulo tecemos alguns comentários sobre os resultados obtidos neste trabalho e apresentamos algumas questões para trabalhos futuros.

\section{Sistemas dinâmicos com um único ponto de equilíbrio hiper- bólico}

1) Na Subseção 2.1 .3 vimos vários exemplos de campos vetoriais em $\mathfrak{X}_{s}^{1}\left(\mathbb{R}^{2}\right)$ que são selas globais porém não estão em $\mathfrak{D}^{1}\left(\mathbb{R}^{2}\right)$, ou seja, cujo divergente não é quase-integrável. De fato na Proposição 2.22 provamos que todo campo $X \in \mathfrak{X}_{s}^{1}\left(\mathbb{R}^{2}\right)$ polinomial quadrático é sela global mesmo que pela Observação 2.21 muitos de tais campos não estejam em $\mathfrak{D}^{1}\left(\mathbb{R}^{2}\right)$. Isto nos motiva a acreditar que talvez seja possível estabelecer um critério para que $X$ seja sela global sem usar tal hipótese (começando pelo caso polinomial) estudando, por exemplo, o comportamento do campo próximo do infinito.

2) Dado $X \in \mathfrak{X}_{s}^{1}\left(\mathbb{R}^{2}\right)$, no Teorema 2.30, apresentamos um invariante, por meio de uma árvore, para o subsistema associado $X$ restrito a $\cup_{i=0}^{3} G_{i}$, onde $G_{i}$ denota a união das regiões canônicas de $\mathcal{F}\left(W_{i}\right)$ inteiramente cobertas pelas separatrizes da sela $O$ de $X$ em rotação (cf. Observação 2.25). Sendo assim, seria interessante encontrar algum critério sobre $X \in \mathfrak{X}_{s}^{1}\left(\mathbb{R}^{2}\right)$ que garantisse que as separatrizes da sela $O$ rotacionadas cobrissem todo o plano, ou seja, que $G_{i}=W_{i}$. Neste caso, o teorema citado acima nos daria um invariante completo para o sistema associado a $X$.

3) Vimos que em [13] Cima et al deram um contra-exemplo polinomial para a conjetura de Markus-Yamabe quando $n \geq 3$. Isto nos leva a crer que no contexto de selas globais, os Teoremas A e B também não devem ser válidos para $n \geq 3$. Porém até o momento não foi possível estabelecer a veracidade desta afirmação. Por outro lado, seria interessante obter condições extras sobre o campo para garantir a tese também para $n \geq 3$.

4) Sobre os resultados de sistemas discretos apresentados na Seção 2.2, vimos no 
Exemplo 2.36 que dado um difeomorfismo $f \in \operatorname{Diff}_{s}^{+}\left(\mathbb{R}^{2}\right)$ com uma das separatrizes $s$ se acumulando só no infinito nem sempre as outras separatrizes de $O$ também se acumulam somente no infinito. Isto revela a importância da separatriz $s$ ser $f$-invariante. Sendo assim, uma proposta para difeomorfismos $f \in D_{i f f_{s}^{+}}\left(\mathbb{R}^{2}\right)$, cujas variedades invariantes não se intersectam, seria buscar o entendimento das seguintes situações: quando uma das separatrizes de $O$ se acumula somente no infinito mas não é $f$-invariante, o que podemos dizer sobre as outras separatrizes de $O$ ? Por exemplo, a hipótese mais simples para uma separatriz que não se acumula no infinito é que seu fecho contenha um ponto dois periódico hiperbólico. Para entender a dinâmica neste caso, é útil considerar o segundo iterado do difeomorfismo. Isto leva ao estudo dos difeomorfismos com exatamente três pontos fixos hiperbólicos, sendo um deles do tipo sela. Este é um problema em aberto, assim como o análogo quando nenhuma das variedades invariantes se acumulam somente no infinito.

\section{Injetividade global}

5) Em [28] os autores provaram que dada uma aplicação $X=\left(f_{1}, f_{2}, f_{3}\right): \mathbb{R}^{3} \rightarrow \mathbb{R}^{3}$, de classe $C^{2}$, a hipótese sobre o espectro de $X$ dada por $\operatorname{Spec}(X) \cap(-\epsilon, \epsilon)=\varnothing$, para algum $\epsilon>0$, implicava na restrição topológica de que cada folheação $\mathcal{F}_{i}$ é por planos. Isto permitiu-lhes obter a seguinte versão fraca da conjetura Jacobiana Real de Jelonek:

Teorema 4.1. Seja $X: \mathbb{R}^{3} \rightarrow \mathbb{R}^{3}$ uma aplicação polinomial e suponha que existe $\epsilon>0$ tal que $\operatorname{Spec}(X) \cap[0, \epsilon)=\varnothing$. Se $\operatorname{codim}\left(S_{X}\right) \geq 2$, então $X$ é uma bijeção.

Isto nos motiva a questionar se seria possível estabelecer também no caso $n$-dimensional alguma hipótese espectral que implicasse na condição topológica adicional do Teorema E. Note que, muito além de uma questão de elegância o ponto aqui é o fato de que, geralmente, é mais fácil estudar o espectro de uma aplicação do que entender suas folheações coordenadas e suas respectivas interseções.

6) No trabalho [27], Gutierrez estabeleceu uma brilhante relação entre a injetividade de um difeomorfismo local do plano $X=\left(f_{1}, f_{2}\right): \mathbb{R}^{2} \rightarrow \mathbb{R}^{2}$, de classe $C^{1}$, e seu espectro, mostrando que a condição $\operatorname{Spec}(X) \cap[0, \infty)=\varnothing$ é suficiente para que $X$ fosse biunívoca. Mais tarde, em [22], esta condição foi enfraquecida para $\operatorname{Spec}(X) \cap[0, \epsilon)=\varnothing$ ou $\operatorname{Spec}(X) \cap$ $(-\epsilon, 0]=\varnothing$, para algum $\epsilon>0$, e o resultado provado para aplicações diferenciáveis (não necessariamente $C^{1}$ ). Esta relação entre injetividade e o espectro de $X$ foi estabelecida através do estudo das folheações $\mathcal{F}_{i}, i=1,2$. Com efeito, não é difícil provar que se $X$ não for injetiva, então existem níveis desconexos para ambas folheações $\mathcal{F}_{i}, i=1,2$, isto é, existem números reais $c_{i}$ tais que $f_{i}^{-1}\left(c_{i}\right)$ é desconexo, para cada $i=1,2$. Em [27], Gutierrez mostrou que a existência de níveis desconexos em $\mathcal{F}_{i}$ implica a existência de semi-componentes de Reeb em $\mathcal{F}_{i}$ (ver [27, 22] para maiores detalhes deste objeto). 
Por fim, ele conclui que a existência de semi-componentes de Reeb é incompatível com qualquer uma das hipóteses espectrais descritas acima. Ressaltamos aqui que um dos passos primordiais nesta cadeia de raciocínio, é o fato de que a não injetividade de $X$ implica na existência de nível desconexo em $\mathcal{F}_{i}$ para $i=1,2$.

Por outro lado, quando aumentamos a dimensão do problema e consideramos um difeomorfismo local $X=\left(f_{1}, \ldots, f_{n}\right): \mathbb{R}^{n} \rightarrow \mathbb{R}^{n}$, de classe $C^{2}$, perde-se cada uma das implicações descritas acima e portanto não é possível concluir que nenhuma das hipóteses espectrais acima implique na injetividade de $X$. De fato, Smith e Xavier provaram em [57] que existem inteiros $n>2$ e aplicações polinomiais não injetivas $P: \mathbb{R}^{n} \rightarrow \mathbb{R}^{n}$ satisfazendo $\operatorname{Spec}(X) \cap[0, \infty]=\varnothing$. Vale lembrar que em [57], os autores não explicitam exemplos, ou seja, eles apresentam um resultado de existência. Além disto, até o presente momento desconhecemos exemplos explícitos satisfazendo estas condições.

Estas considerações nos levam a questionar se seria possível estabelecer alguma relação entre a não injetividade de $X$ e alguma propriedade das folheações $\mathcal{F}_{i}, i=1, \ldots, n$, análoga à existência de nível desconexo no caso do plano. No caso particular em que $n=3$, temos por [28] que se $\operatorname{Spec}(X) \cap(-\epsilon, \epsilon)=\varnothing$, para algum $\epsilon>0$, então as folheações $\mathcal{F}_{i}$ são por planos. Se $X$ não for injetiva, o que podemos dizer sobre as folheações $\mathcal{F}_{i}$ ? Acreditamos que o entendimento deste passo fundamental, poderia dar pistas de como trabalhar no problema 5) acima, assim como em conjeturas sobre injetividade, por exemplo, a de Nollet e Xavier, apresentada no capítulo anterior e a conjetura de Chamberland que afirma que se $X: \mathbb{R}^{n} \rightarrow \mathbb{R}^{n}$ é uma aplicação de classe $C^{1}$ tal que, para algum $\epsilon>0$, $\operatorname{Spec}(X) \cap\{z \in \mathbb{C} ;|z|<\epsilon\}=\varnothing$, então $X$ é injetiva. É claro que, de maneira indireta, isto nos ajudaria a entender um pouco mais sobre a relação entre folheações e a conjetura Jacobiana, uma vez que a veracidade da última conjetura acima implica que a conjetura Jacobiana é verdadeira. 


\section{BIBLIOGRAFIA}

[1] B. Alarcón, V. Guiñéz, and C. Gutierrez. Hopf bifurcation at infinity for planar vector fields. Discrete and Continuous Dynamical Systems, 17(2):247-258, 2007.

[2] B. Alarcón, V. Guiñéz, and C. Gutierrez. Planar embeddings with a globally attracting fixed point. Nonlinear Analysis series A: Theory, Methods and Aplications, 69(1):140-150, 2008.

[3] B. Alarcón, C. Gutierrez, and J. Martínez-Alfaro. Planar maps whose second iterate has a unique fixed point. J. of Difference Equations and Applications, 14(4):421-428, 2008.

[4] J. Artés, F. Durmotier, J. Llibre. Qualitative theory of planar differential systems. Springer-Verlag, Berlin Heidelberg, 2006.

[5] E. Balreira. Foliations and global inversion. Commentarii Math. Helvetici, 85(1):7383, 2010.

[6] J. Bernat and J. Llibre. Counterexamples to Kalman and Markus-Yamabe conjectures in dimension larger than 3. Dyn. Contin. Discrete Impuls. Systems, 2:337-379, 1996.

[7] N. P. Bhatia and G. P. Szegö. Stability Theory of Dynamical Systems. Classics in Math., Springer-Verlag, Berlin, 2002.

[8] A. Bialynicki-Birula and M. Rosenlicht. Injective morphisms of real varieties. Proc. Am. Math. Soc., 13:200-203, 1962.

[9] F. Braun and J. R. dos Santos Filho. The real Jacobian conjecture on $\mathbb{R}^{2}$ is true when one of the components has degree 3. Discrete and Continuous Dynamical Systems, 26(1):75-87, 2010. 
[10] C. Camacho and A. Lins Neto. Geometric theory of foliations. Boston, Birkhäuser, 1985.

[11] A. Candel and L. Colon. Foliations I. Graduates Studies in Math., AMS, 2000.

[12] A. Candel and L. Colon. Foliations II. Graduates Studies in Math., AMS, 2003.

[13] A. Cima, A. van de Essen, A. Gasull, E. Hubbers, and F. Mañosas. A polynomial counterexample to the Markus-Yamabe Conjecture. Adv. Math., 131:453-457, 1997.

[14] A. Cima, A. Gasull, and F. Mañosas. The discrete Markus-Yamabe problem. Nonlinear Analysis, 35:343-354, 1999.

[15] M. Cobo, C. Gutierrez, and J. Llibre. On the injectivity of $C^{1}$ maps of the real plane. Canad. J. Math., 54(6):1187-1201, 2002.

[16] B.A. Coomes. On condition sufficient for injectivity of maps. IMA Preprint Series $544,1989$.

[17] k. Drużkowisk and H. Tutaj. Differential conditions to verify the Jacobian Conjecture. Ann. Polon. Math., 46:85-90, 1992.

[18] G.F.D. Duff. Limit cycles and rotated vector fields. Ann. Math., 57:15-31, 1953.

[19] A. van de Essen. Conjectures and problems surrounding the Jacobian Conjecture. Report 9345, University of Nijmegen, 1993.

[20] A. van de Essen and E. Hubbers. A new class of invertible polynomial maps. J. Algebra, 187:214-226, 1997.

[21] A. Fernandes, C. Gutierrez, and R. Rabanal. On local diffeomorphisms of $\mathbb{R}^{n}$ that are injective. Qual. Theory Dyn. Syst., 4(2):255-262, 2004.

[22] A. Fernandes, C. Gutierrez, and R. Rabanal. Global asymptotic stability for differentiable vector fields of $\mathbb{R}^{2}$. J. Differential Equations, 206(2):470-482, 2004.

[23] R. Fessler. A proof of the two-dimensional Markus-Yamabe stability conjecture and a generalization. Ann. Polon. Math., 62(1):45-74, 1995.

[24] A. Gasull, J. Llibre, and J. Sotomayor. Global asymptotic stability of differential equations in the plane. J. Differential Equations, 91:327-335, 1991.

[25] H. Giacomini and M. Grau. On the stability of limit cycles for planar differential systems. J. Differential Equations, 213(2):368-388, 2005. 
[26] A. A. Glutsyuk. A complete solution of the Jacobian problem for vector fields on the plane. Russian Math. Surveys, 49:627-671, 1995.

[27] C. Gutierrez. A solution to the bidimensional global asymptotic stability conjecture. Ann. Inst. H. Poincaré Anal. Non Linéaire, 12(6):627-671, 1995.

[28] C. Gutierrez and C. Maquera. Foliations and polynomial diffeomorphisms of $\mathbb{R}^{3}$. Math. Zeitschrift, 262:613-626, 2009.

[29] C. Gutierrez, J. Martinez-Alfaro, and J. Venato-Santos. Plane foliations with a saddle singularity. to appear at Proc. of the 1st Workshop on Singularities in Generic Geometry and Applications, by Topology and its Applications.

[30] C. Gutierrez, B. Pires, and R. Rabanal. Asymptotic Stability at infinity for differentiable vector fields of the plane. J. Differential Equations, 231:165-181, 2006.

[31] C. Gutierrez and R. Rabanal. Injectivity of differentiable maps $\mathbb{R}^{2} \rightarrow \mathbb{R}^{2}$ at infinity. Bull. Braz. Math. Soc., 37(2):217-239, 2006.

[32] C. Gutierrez and A. Sarmiento. Injectivity of $C^{1}$ maps $\mathbb{R}^{2} \rightarrow \mathbb{R}^{2}$ at infinity and planar vector fields. Astérisque, (287):xviii, 89-102, 2003. Geometric methods in dynamics. II.

[33] C. Gutierrez and M. A. Teixeira. Asymptotic stability at infinity of planar vector fields. Bol. Soc. Brasil. Mat. (N.S.), 26(1):57-66, 1995.

[34] J. Gwozdziewicz and Z. Jelonek. A geometry of real polynomial mappings of the plane and the Pinchuk example. IMUJ Preprint, Kraków, 1998.

[35] P. Hartman. Ordinary differential equations. S. M. Hartman, Baltimore, Md., 1973.

[36] Z. Jelonek. Geometry of real polynomial mappings. Math. Zeitschrift, 239:321-333, 2002 .

[37] W. Kaplan. Regular curve-families filling the plane, I. Duke Math. J., 7:154-155, 1940. II. Duke Math. J., 8:11-46, 1941.

[38] O. H. Keller. Ganze cremona-transformationen. Monatsh. Math. Phys., 47:299-306, 1939.

[39] L. Markus. Global structure of ordinary differential equations in the plane. Trans. Amer. Math. Soc., 76:127-148, 1954. 
[40] L. Markus and H. Yamabe. Global stability criteria for diferential systems. Osaka J. Math., 12:305-317, 1960.

[41] G. H. Meisters and C. Olech. Global stability, injectivity, and the Jacobian conjecture. Proceedings, First World Congress of Nonlinear Analysts, Tampa, Florida, 1059-1072, 1991.

[42] W. de Melo and J. Palis. Introdução aos sistemas dinâmicos. Projeto Euclides, IMPA, Rio de Janeiro, 1978.

[43] J. Milnor. Morse theory. Princenton University Press, 1963.

[44] C. Morales and B. Scárdua. Geometry, Dynamics and Topology of Foliated Manifolds. Publicações Matemáticas, IMPA, Rio de Janeiro, 2003.

[45] S. Nollet and F. Xavier. Global inversion via the Palais-Smale conditions. Discrete and Continuous Dynamical Systems, 8:17-28, 2002.

[46] C. Olech. On the global stability of an autonomous system on the plane. Contributions to Differential Equations, 1:389-400, 1963.

[47] T. Parthasarathy. On global univalence theorems. volume 977 of Lecture Notes in Mathematics. Springer-Verlag, 1983.

[48] L. M. Perko. Rotated vector fields. J. Differential Equations, 103:127-145, 1993.

[49] S. Pinchuck. A counterexample to the strong Jacobian conjecture. Math. Zeitschrift, 217:1-4, 1994.

[50] B. Pires and R. Rabanal. Vector fields whose linearisation is Hurwitz almost everywhere. arXiv:1102.0190v1, 2011.

[51] R. Rabanal. Center type performance differentiable vector fields in the plane. Proc. Amer. Math. Soc., 137(2):653-662, 2008.

[52] J. Reeb. Sur certains propriétés topologiques des variétés feuilletées. Actual Sci. Ind., 1183, Hermann, Paris, 1952.

[53] J. Reeb. Les espaces localement numériques non separés et leurs applications à un problème classique. Coll. top. Strasbourg, 1955.

[54] C. Robinson. Dynamical systems: stability, symbolic dynamics, and chaos. CRC Press, 2a ed., 1999. 
[55] R. Sacksteder. Sur Foliations and pseudogroups. Amer. J. Math., 87:79-170, 1965.

[56] S. Smale. Mathematical problems for the next century. Math. Intelligencer, 20:7-15, 1998.

[57] B. Smyth and F. Xavier. Injectivity of local diffeomorphisms from nearly spectral conditions. J. Differential Equations, 130:406-414, 1996.

[58] J. Sotomayor. Lições de equações diferenciais ordinárias. Projeto Euclides, IMPA, Rio de Janeiro, 1979.

[59] E.H. Spanier. Algebraic Topology. MacGraw-Hill, New York, 1969.

[60] X. Wang. On the $C^{*}$-Algebras of Foliations in the Plane, Lecture Notes in Mathematics 125\%. Springer-Verlag, Berlin Heidelberg, 1987. 


\section{ÍNDICE}

$E^{s}, 11$

$E^{u}, 11$

$F(\theta), 37$

$G_{i}, 40$

$L_{i}, 40$

$S_{X}, 7,52$

$W_{i}, 5,23,40$

$\mathfrak{X}^{1}(U), 3,9$

$\mathfrak{D}^{1}(U), 27$

$\mathfrak{X}_{s}^{1}\left(\mathbb{R}^{2}\right), 3$

$\operatorname{Diff}_{s}^{+}\left(\mathbb{R}^{2}\right), 6,44$

$\operatorname{Diff}_{s}\left(\mathbb{R}^{2}\right), 6,44$

$\operatorname{Div}(X), 4,27$

$\mathcal{F}, 14$

$\mathcal{F}_{i}, 49$

$\mathcal{F}_{i_{1} \ldots i_{k}}, 49$

$\mathcal{F}\left(W_{i}\right), 23,40$

$\operatorname{Fix}(f), 45$

$\mathcal{F}\left(G_{i}\right), 40$

$\Omega(f), 20$

$\mathcal{S}, 6,45$

$\mathcal{S S}, 30$

$\operatorname{Spec}(X), 1,30$

$l_{\theta}^{i}, 38$

Aplicação

de holonomia, 15

de Morse, 16

própria, 52

Atrator global, 2, 6

Campo vetorial, 9
Campos vetoriais em rotação, 37

Centro global, 3

Ciclo limite, 12

Configuração de separatrizes, 13

Conjetura

discreta de Markus-Yamabe, 6

de Chamberland, 65

de Markus-Yamabe, 2, 30

de Nollet e Xavier, 8, 61

Jacobiana, 8, 53

Jacobiana real de Jelonek, 7, 52

Conjugação topológica

de difeomorfismos, 20

Conjunto

$\alpha$-limite, 11,20

$\gamma_{p+}, 11$

$\gamma_{p-}, 11$

$\omega$-limite, 11 , 20

minimal, 15

saturado, 15

semi-algébrico, 52

Difeomorfismo

que preserva orientação, 21

que reverte orientação, 21

Equivalência topológica

de campos, 10

de folheações, 14

Espaço das folhas, 14

Fluxo, 10 
Folheações, 14

folhas de, 14

placas de, 14

Função

quase-integrável, 3, 26

Grupo de holonomia, 15

Índice de estabilidade, 10,21

Lema

de Morse, 16

Órbita, 9, 20

Ponto

crítico, 16

crítico não degenerado, 16

de equilíbrio, 1, 10

fixo, 1, 20

fixo hiperbólico, 20

não errante, 20

não próprio, 7. 51

periódico, 20

regular, 10

singular, 1, 10

singular hiperbólico, 10

Posição geral, 17

Raio $f$-invariante, 45

Região

paralela, 12

Região canônica

de nível $n, 25$

de um campo, 13

de uma folheação do plano, 19

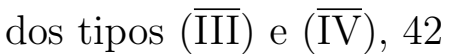

inicial, 24

Regiões canônicas adjacentes, 19

Sela global, 4, 29
Sela hiperbólica, 10, 21

Separatriz

de ponto fixo, 44

de um campo, 12

de uma folheação do plano, 18

estável de um ponto singular, 12

inicial, 24, 25

instável de um ponto singular, 12

limite de um campo, 13

Setor pseudo-hiperbólico no $\infty, 25$

Setor pseudo-hiperbólico inicial no $\infty, 40$

Sistemas dinâmicos

contínuos, 1

discretos, 1

Teorema

da estabilidade local de Reeb, 15

de Poincaré-Bendixon, 12

das Variedades estável e instável, 11

de Hartman-Grobman, 10, 21

Topologia grossa das folhas, 18

Trajetória, 9

Variedade

estável, 21

estável local, 11

instável, 21

instável local, 11 